\title{
AUBERT DUALS OF DISCRETE SERIES: THE FIRST INDUCTIVE STEP
}

\author{
IVAN MATIĆ \\ University of Osijek, Croatia
}

\begin{abstract}
Let $G_{n}$ denote either symplectic or odd special orthogonal group of rank $n$ over a non-archimedean local field $F$. We provide an explicit description of the Aubert duals of irreducible representations of $G_{n}$ which occur in the first inductive step in the realization of discrete series representations starting from the strongly positive ones. Our results might serve as a pattern for determination of Aubert duals of general discrete series of $G_{n}$ and should produce an interesting part of the unitary dual of this group. Furthermore, we obtain an explicit form of some representations which are known to be unitarizable.
\end{abstract}

\section{INTRODUCTION}

Let $F$ denote a non-archimedean local field and let $G_{n}$ stand for either symplectic or odd special orthogonal group of rank $n$ over $F$. This paper presents a continuation of our previous work on the explicit determination of the Aubert duals of irreducible admissible representations of the group $G_{n}$. The involution on the Grothendieck group of the smooth finite-length representations of a reductive group has been studied by many authors, and we use an involution defined for general reductive $p$-adic groups in [2]. This involution is known as the Aubert involution and the image of a representation under this involution is called the Aubert dual of a representation. The Aubert involution can be used as a powerful method for studying the induced representations of certain type, as has been done in [7, 27]. Also, it corresponds to the interchange of the two copies of the special linear group $S L(2, \mathbb{C})$ in local Arthur parameters, i.e., the local Arthur parameters of an irreducible representation and its Aubert dual are symmetric. Although this

2010 Mathematics Subject Classification. 22E35, 22E50, 11F70.

Key words and phrases. Aubert dual, discrete series, classical $p$-adic groups. 
involution satisfies many remarkable properties, an explicit description of the Aubert duals of irreducible representations is known only for a small class of representations. Our intention is to extend this knowledge to a certain class of discrete series representations.

By the Mœglin-Tadić classification of discrete series, every non-cuspidal discrete series representation of $G_{n}$ can be obtained in a finite number of steps starting from a strongly positive discrete series of $G_{n^{\prime}}$, for some $n^{\prime} \leq n$. Each of these steps consists of a parabolic induction with respect to a maximal parabolic subgroup from a representation which has an appropriate irreducible essentially square-integrable representation on the general linear group part and a previous discrete series representation on the classical group part. In terms of this classification, each step consists of adding two consecutive elements in the Jordan block of a discrete series representation of smaller rank group of the same type (the Jordan block is one of the parameters attached to a discrete series by this classification). For more details regarding this construction we refer the reader to [20] and [22]. However, to keep the notation as simple as possible, throughout the paper we do not follow the notation used in [22] and rather use an algebraic formulation when introducing a discrete series representation.

We emphasize that the classification of discrete series given in $[20,22]$ now holds unconditionally, due to results of [1], [21, Théorème 3.1.1] and [4, Theorem 7.8]. A shorter form of this classification, which covers both classical and odd general spin groups, can be found in [9].

In our previous paper ([17]), we obtained an explicit description of the Aubert duals of strongly positive discrete series representations of $G_{n}$. The natural following step is to investigate the Aubert duals of discrete series representations obtained by adding two consecutive elements in the Jordan block of a strongly positive representation. Such a construction presents the first inductive step in the Møglin-Tadić classification, and provides much more complicated discrete series than the strongly positive ones. We note that such discrete series have also played a crucial role in the determination of the first occurrence indices of discrete series in the metaplectic odd-orthogonal tower ([13]). Also, in the generalized principal series induced from such a discrete series on the classical group part occur much more complicated situations then in those induced from the strongly positive ones ([15]).

To determine the Aubert duals of discrete series appearing in the first inductive step of the mentioned classification, we use essentially the same methods as in [17]. Our approach is based on the knowledge on the Jacquet modules of studied discrete series, obtained in [14] and [16], enhanced by the intertwining operators method and basic properties of the Aubert involution. This enables us to determine some prominent members appearing in the Jacquet modules of the Aubert duals, and then to provide an explicit description of the Aubert duals from their Jacquet modules. Since the Jacquet 
modules of discrete series which we study are much more involved than those of the strongly positive ones, we introduce the notion of so-called $(\sigma, \widehat{\sigma})$-triple, which enables us to use an inductive procedure when determining the Aubert duals. We note that similar methods have been used in [18] to describe the Langlands quotients of the generalized principal series which are fixed under the Aubert involution.

In the case of general linear groups, an algorithm for the explicit determination of the Aubert duals is given in [23], and later, by a more explicit formula, in [10]. Also, we take a moment to note the recent paper of Jantzen ([8]), which provides an algorithm for classical groups in the half-integral case.

An important conjecture regarding the Aubert involution states that it preserves unitarizability, so our results should provide a class of unitarizable representations. Furthermore, results of $[5,25]$ show that the obtained representations are in particular cases already known to be unitarizable.

Let us now describe the content of the paper in more details. In the second section we recall the required notations and preliminaries. In the third section we prove some technical results which are used later in the paper. A description of the Aubert duals in the most complicated cases is provided in the fourth section, using a case-by-case consideration. The exceptional cases are discussed in the fifth section.

The author would like to thank the referees for a number of corrections and very useful suggestions.

This work has been supported by Croatian Science Foundation under the project 9364 .

\section{Preliminaries}

Throughout the paper, $F$ will denote a non-archimedean local field of characteristic different than two.

For a connected reductive $p$-adic group $G$ defined over field $F$, let $\Sigma$ denote the set of roots of $G$ with respect to fixed minimal parabolic subgroup and let $\Delta$ stand for a basis of $\Sigma$. For $\Theta \subseteq \Delta$, we let $P_{\Theta}$ denote the standard parabolic subgroup of $G$ corresponding to $\Theta$ and let $M_{\Theta}$ denote a corresponding standard Levi subgroup. Let $W$ denote the Weyl group of $G$.

For a parabolic subgroup $P$ of $G$ with the Levi subgroup $M$, and a representation $\sigma$ of $M$, we denote by $i_{M}(\sigma)$ a normalized parabolically induced representation of $G$ induced from $\sigma$. Also, let $r_{M}(\sigma)$ stand for the normalized Jacquet module of an admissible finite length representation $\sigma$ of $G$, with respect to the standard parabolic subgroup having the Levi subgroup equal to $M$.

We take a moment to recall the definition of the Aubert involution and some of its basic properties $([2,3])$. 
THEOREM 2.1. Define the operator on the Grothendieck group of admissible representations of finite length of $G$ by

$$
D_{G}=\sum_{\Theta \subseteq \Delta}(-1)^{|\Theta|} i_{M_{\Theta}} \circ r_{M_{\Theta}} .
$$

Operator $D_{G}$ has the following properties:

(i) $D_{G}$ is an involution.

(ii) $D_{G}$ takes irreducible representations to irreducible ones.

(iii) If $\sigma$ is an irreducible cuspidal representation, then $D_{G}(\sigma)=(-1)^{|\Delta|} \sigma$.

(iv) For a standard Levi subgroup $M=M_{\Theta}$, we have

$$
r_{M} \circ D_{G}=A d(w) \circ D_{w^{-1}(M)} \circ r_{w^{-1}(M)},
$$

where $w$ is the longest element of the set $\left\{w \in W: w^{-1}(\Theta)>0\right\}$.

(v) For a standard Levi subgroup $M=M_{\Theta}$, we have $D_{G} \circ i_{M}=i_{M} \circ D_{M}$.

Let us now describe the groups that we consider. We look at the usual towers of symplectic or orthogonal groups $G_{n}=G\left(V_{n}\right)$ that are groups of isometries of $F$-spaces $\left(V_{n},(),\right), n \geq 0$, where the form $($,$) is non-degenerate$ and it is skew-symmetric if the tower is symplectic and symmetric otherwise. The set of standard parabolic subgroups of the group $G_{n}$ will be fixed in the usual way.

Then the Levi subgroups of standard parabolic subgroups have the form $M \cong G L\left(n_{1}, F\right) \times \cdots \times G L\left(n_{k}, F\right) \times G_{n^{\prime}}$, where $G L\left(n_{i}, F\right)$ denotes a general linear group of rank $n_{i}$ over $F$. For simplicity of exposition, if $\delta_{i}, i=1,2, \ldots, k$ denotes a representation of $G L\left(n_{i}, F\right)$, and if $\tau$ stands for a representation of $G_{m}$, we let $\delta_{1} \times \delta_{2} \times \cdots \times \delta_{k} \rtimes \tau$ stand for the induced representation $i_{M}\left(\delta_{1} \otimes\right.$ $\left.\delta_{2} \otimes \cdots \otimes \delta_{k} \otimes \tau\right)$ of $G_{n}$, where $M$ is the standard Levi subgroup isomorphic to $G L\left(n_{1}, F\right) \times \cdots \times G L\left(n_{k}, F\right) \times G_{m}$. Here $n=n_{1}+n_{2}+\cdots+n_{k}+m$.

Similarly, by $\delta_{1} \times \delta_{2} \times \cdots \times \delta_{k}$ we denote the induced representation $i_{M^{\prime}}\left(\delta_{1} \otimes \delta_{2} \otimes \cdots \otimes \delta_{k}\right)$ of the group $G L\left(n^{\prime}, F\right)$, where the Levi subgroup $M^{\prime}$ equals $G L\left(n_{1}, F\right) \times G L\left(n_{2}, F\right) \times \cdots \times G L\left(n_{k}, F\right)$ and $n^{\prime}=n_{1}+n_{2}+\cdots+n_{k}$.

Let $\operatorname{Irr}(G L(n, F))$ denote the set of all irreducible admissible representations of $G L(n, F)$, and let $\operatorname{Irr}\left(G_{n}\right)$ denote the set of all irreducible admissible representations of $G_{n}$. Let $R(G L(n, F))$ stand for the Grothendieck group of admissible representations of finite length of $G L(n, F)$ and define $R(G L)=\oplus_{n \geq 0} R(G L(n, F))$. Similarly, let $R\left(G_{n}\right)$ stand for the Grothendieck group of admissible representations of finite length of $G_{n}$ and define $R(G)=$ $\oplus_{n \geq 0} R\left(G_{n}\right)$.

If $\sigma$ is an irreducible representation of $G_{n}$, we denote by $\hat{\sigma}$ the representation $\pm D_{G_{n}}(\sigma)$, taking the sign + or - such that $\hat{\sigma}$ is a positive element in $R\left(G_{n}\right)$. We call $\hat{\sigma}$ the Aubert dual of $\sigma$.

Using Jacquet modules for the maximal standard parabolic subgroups of $G L(n, F)$, we can define $m^{*}(\pi)=\sum_{k=0}^{n}\left(r_{(k)}(\pi)\right) \in R(G L) \otimes R(G L)$, for an irreducible representation $\pi$ of $G L(n, F)$, and then extend $m^{*}$ linearly to the 
whole of $R(G L)$. Here $r_{(k)}(\pi)$ denotes the normalized Jacquet module of $\pi$ with respect to the standard parabolic subgroup having the Levi subgroup equal to $G L(k, F) \times G L(n-k, F)$, and we identify $r_{(k)}(\pi)$ with its semisimplification in $R(G L(k, F)) \otimes R(G L(n-k, F))$.

Let us denote by $\nu$ the composition of the determinant mapping with the normalized absolute value on $F$. Let $\rho \in \operatorname{Irr}(G L(k, F))$ denote a cuspidal representation. By a segment of cuspidal representations we mean a set of the form $\left\{\rho, \nu \rho, \ldots, \nu^{m} \rho\right\}$, which we denote by $\left[\rho, \nu^{m} \rho\right]$.

The results of [29] show that each irreducible essentially square-integrable representation $\delta \in \operatorname{Irr}(G L(n, F))$ is attached to a segment, and we set $\delta=$ $\delta\left(\left[\nu^{a} \rho, \nu^{b} \rho\right]\right)$, which is the unique irreducible subrepresentation of $\nu^{b} \rho \times \nu^{b-1} \rho \times$ $\cdots \times \nu^{a} \rho$, where $a, b \in \mathbb{R}$ are such that $b-a$ is a non-negative integer and $\rho$ is an irreducible unitary cuspidal representation of some $G L(k, F)$.

We frequently use the following equation:

$$
m^{*}\left(\delta\left(\left[\nu^{a} \rho, \nu^{b} \rho\right]\right)\right)=\sum_{i=a-1}^{b} \delta\left(\left[\nu^{i+1} \rho, \nu^{b} \rho\right]\right) \otimes \delta\left(\left[\nu^{a} \rho, \nu^{i} \rho\right]\right) .
$$

Note that multiplicativity of $m^{*}$ implies

$m^{*}\left(\prod_{j=1}^{n} \delta\left(\left[\nu^{a_{j}} \rho_{j}, \nu^{b_{j}} \rho_{j}\right]\right)\right)=\prod_{j=1}^{n}\left(\sum_{i_{j}=a_{j}-1}^{b_{j}} \delta\left(\left[\nu^{i_{j}+1} \rho_{j}, \nu^{b_{j}} \rho_{j}\right]\right) \otimes \delta\left(\left[\nu^{a_{j}} \rho_{j}, \nu^{i_{j}} \rho_{j}\right]\right)\right)$.

For a representation $\sigma \in R\left(G_{n}\right)$ and $1 \leq k \leq n$, we denote by $r_{(k)}(\sigma)$ the normalized Jacquet module of $\sigma$ with respect to the parabolic subgroup $P_{(k)}$ having the Levi subgroup equal to $G L(k, F) \times G_{n-k}$. We identify $r_{(k)}(\sigma)$ with its semisimplification in $R(G L(k, F)) \otimes R\left(G_{n-k}\right)$ and consider

$$
\mu^{*}(\sigma)=1 \otimes \sigma+\sum_{k=1}^{n} r_{(k)}(\sigma) \in R(G L) \otimes R(G) .
$$

We take a moment to state a result, derived in [26], which presents a crucial structural formula for our calculations of Jacquet modules of classical groups.

LEMMA 2.2. Let $\rho \in \operatorname{Irr}(G L(n, F))$ denote a cuspidal representation and let $k, l \in \mathbb{R}$ such that $k+l$ is a non-negative integer. Let $\sigma \in R(G)$ be an admissible representation of finite length. Write $\mu^{*}(\sigma)=\sum_{\tau, \sigma^{\prime}} \tau \otimes \sigma^{\prime}$. Then the following holds:

$$
\begin{aligned}
\mu^{*}\left(\delta\left(\left[\nu^{-k} \rho, \nu^{l} \rho\right]\right) \rtimes \sigma\right)= & \sum_{i=-k-1}^{l} \sum_{j=i}^{l} \sum_{\tau, \sigma^{\prime}} \delta\left(\left[\nu^{-i} \widetilde{\rho}, \nu^{k} \widetilde{\rho}\right]\right) \times \delta\left(\left[\nu^{j+1} \rho, \nu^{l} \rho\right]\right) \times \tau \otimes \\
& \otimes \delta\left(\left[\nu^{i+1} \rho, \nu^{j} \rho\right]\right) \rtimes \sigma^{\prime} .
\end{aligned}
$$

We omit $\delta\left(\left[\nu^{x} \rho, \nu^{y} \rho\right]\right)$ if $x>y$. 
We briefly recall the Langlands classification for general linear groups. We favor the subrepresentation version of this classification over the quotient one.

For every irreducible essentially square-integrable representation $\delta \in$ $R(G L)$, there is a unique $e(\delta) \in \mathbb{R}$ such that $\nu^{-e(\delta)} \delta$ is unitarizable. Note that $e\left(\delta\left(\left[\nu^{a} \rho, \nu^{b} \rho\right]\right)\right)=(a+b) / 2$. Suppose that $\delta_{1}, \delta_{2}, \ldots, \delta_{k}$ are irreducible essentially square-integrable representations such that $e\left(\delta_{1}\right) \leq e\left(\delta_{2}\right) \leq \ldots \leq e\left(\delta_{k}\right)$. Then the induced representation $\delta_{1} \times \delta_{2} \times \cdots \times \delta_{k}$ has a unique irreducible subrepresentation, which we denote by $L\left(\delta_{1}, \delta_{2}, \ldots, \delta_{k}\right)$. This irreducible subrepresentation is called the Langlands subrepresentation, and it appears with multiplicity one in the composition series of $\delta_{1} \times \delta_{2} \times \cdots \times \delta_{k}$. Every irreducible representation $\pi \in R(G L)$ is isomorphic to some $L\left(\delta_{1}, \delta_{2}, \ldots, \delta_{k}\right)$ and, for a given $\pi$, the representations $\delta_{1}, \delta_{2}, \ldots, \delta_{k}$ are unique up to a permutation among those $\delta_{i}$ having the same exponents.

Similarly, throughout the paper we use the subrepresentation version of the Langlands classification for classical groups, since it is more appropriate for our Jacquet module considerations. So, we realize a non-tempered irreducible representation $\pi$ of $G_{n}$ as the unique irreducible (Langlands) subrepresentation of an induced representation of the form $\delta_{1} \times \delta_{2} \times \cdots \times \delta_{k} \rtimes \tau$, where $\tau$ is a tempered representation of some $G_{t}$, and $\delta_{1}, \delta_{2}, \ldots, \delta_{k} \in R(G L)$ are irreducible essentially square-integrable representations such that $e\left(\delta_{1}\right) \leq$ $e\left(\delta_{2}\right) \leq \cdots \leq e\left(\delta_{k}\right)<0$. In this case, we write $\pi=L\left(\delta_{1}, \delta_{2}, \ldots, \delta_{k}, \tau\right)$.

An irreducible representation $\sigma \in R(G)$ is called strongly positive if for every embedding

$$
\sigma \hookrightarrow \nu^{s_{1}} \rho_{1} \times \nu^{s_{2}} \rho_{2} \times \cdots \times \nu^{s_{k}} \rho_{k} \rtimes \sigma_{\text {cusp }},
$$

where $\rho_{i} \in R\left(G L\left(n_{\rho_{i}}, F\right)\right), i=1,2, \ldots, k$, are cuspidal unitary representations and $\sigma_{\text {cusp }} \in R(G)$ is an irreducible cuspidal representation, we have $s_{i}>0$ for each $i$.

For simplicity of exposition, in this paper we are interested in determining the Aubert duals of certain discrete series whose cuspidal supports consist of twists of one selfcontragredient irreducible cuspidal representation of a general linear group and a cuspidal representation of some $G_{n}$. A description of the Aubert duals of discrete series of the same type and with more general cuspidal supports can then be deduced using [17, Theorem 3.6].

Thus, let $\rho \in \operatorname{Irr}\left(G L\left(n_{\rho}, F\right)\right)$ and $\sigma_{\text {cusp }} \in \operatorname{Irr}\left(G_{n^{\prime}}\right)$ denote cuspidal representations. There is a unique non-negative real number $\alpha$ such that $\nu^{\alpha} \rho \rtimes \sigma_{\text {cusp }}$ reduces, and it follows from [1] and [21, Théorème 3.1.1] that $\alpha$ is a halfinteger.

Now we recall the description of the strongly positive discrete series obtained in $[20,22]$ and in [12].

If $\alpha=0$, then the only strongly positive discrete series with the cuspidal support consisting of $\sigma_{\text {cusp }}$ and of twists of $\rho$ is $\sigma_{\text {cusp }}$. 
Now assume $\alpha>0$ and let $k=\lceil\alpha\rceil$, the smallest integer which is not smaller than $\alpha$. For every strongly positive discrete series $\sigma$ whose cuspidal support entirely consists of $\sigma_{\text {cusp }}$ and of twists of $\rho$, there exist unique real numbers $a_{1}, a_{2}, \ldots, a_{k}$ such that $-1<a_{1}<a_{2}<\cdots<a_{k}, a_{i}-\alpha$ is an integer for $i=1,2, \ldots, k$, and such that $\sigma$ is the unique irreducible subrepresentation of the induced representation

$$
\delta\left(\left[\nu^{\alpha-\lceil\alpha\rceil+1} \rho, \nu^{a_{1}} \rho\right]\right) \times \delta\left(\left[\nu^{\alpha-\lceil\alpha\rceil+2} \rho, \nu^{a_{2}} \rho\right]\right) \times \cdots \times \delta\left(\left[\nu^{\alpha} \rho, \nu^{a_{k}} \rho\right]\right) \rtimes \sigma_{\text {cusp }} .
$$

In the rest of the paper, we denote such representation $\sigma$ by $S P\left(a_{1}, a_{2}, \ldots, a_{k}\right)$.

In the following several results we summarize the first inductive step in the construction of discrete series starting from the strongly positive ones, as in $[22,28]$, and describe the representations whose Aubert duals we study.

TheOREm 2.3. Suppose that $\alpha>0$. The induced representation

$$
\delta\left(\left[\nu^{-a_{j}} \rho, \nu^{a_{j+1}} \rho\right]\right) \rtimes S P\left(a_{1}, \ldots, a_{j-1}, a_{j+2}, \ldots, a_{k+2}\right),
$$

where $-1<a_{1}<a_{2}<\cdots<a_{k+2}$ such that $\alpha-a_{i}$ is an integer for $i=$ $1,2, \ldots, k+2$ and $a_{j} \geq 0$, has exactly two irreducible subrepresentations which are mutually non-isomorphic and square-integrable. We denote them by $\sigma_{1}$ and $\sigma_{2}$. If $j \geq 2$ and $a_{1} \geq 0$ if $j=2$, there is a unique $i \in\{1,2\}$ such that $\sigma_{i}$ is a subrepresentation of the induced representation

$$
\delta\left(\left[\nu^{-a_{j-1}} \rho, \nu^{a_{j}} \rho\right]\right) \rtimes S P\left(a_{1}, \ldots, a_{j-2}, a_{j+1}, \ldots, a_{k+2}\right) .
$$

Also, if $j<k+1$, then $\sigma_{i^{\prime}}$, for $i^{\prime}$ such that $\left\{i, i^{\prime}\right\}=\{1,2\}$, is a subrepresentation of the induced representation

$$
\delta\left(\left[\nu^{-a_{j+1}} \rho, \nu^{a_{j+2}} \rho\right]\right) \rtimes S P\left(a_{1}, \ldots, a_{j}, a_{j+3}, \ldots, a_{k+2}\right) .
$$

Furthermore, for $i=1,2$, there is a unique irreducible tempered subrepresentation $\tau$ of $\delta\left(\left[\nu^{-a_{j}} \rho, \nu^{a_{j}} \rho\right]\right) \rtimes S P\left(a_{1}, \ldots, a_{j-1}, a_{j+2}, \ldots, a_{k+2}\right)$ such that $\sigma_{i}$ is a subrepresentation of $\delta\left(\left[\nu^{a_{j}+1} \rho, \nu^{a_{j+1}} \rho\right]\right) \rtimes \tau$.

Aubert duals of representations described in previous theorem are determined in Section 4. For $a_{j+1}-a_{j} \in\{1,2\}$, it follows from [5, Theorem 3.1, Theorem 3.2] and [6] that the Aubert duals of both representations $\sigma_{1}$ and $\sigma_{2}$ are unitarizable.

Theorem 2.4. Suppose that $\alpha=0$ and let $a, b$ denote non-negative integers such that $a<b$. The induced representation

$$
\delta\left(\left[\nu^{-a} \rho, \nu^{b} \rho\right]\right) \rtimes \sigma_{\text {cusp }}
$$

has exactly two irreducible subrepresentations which are mutually non-isomorphic and square-integrable. We denote them by $\sigma_{1}$ and $\sigma_{2}$. In $R(G)$ we have $\rho \rtimes \sigma_{\text {cusp }}=\tau_{1}+\tau_{-1}$, for irreducible and not isomorphic tempered representations $\tau_{1}$ and $\tau_{-1}$. For every $i \in\{1,2\}$, there is a unique $j \in\{1,-1\}$ such that $\sigma_{i}$ is a subrepresentation of

$$
\delta\left(\left[\nu \rho, \nu^{a} \rho\right]\right) \times \delta\left(\left[\nu \rho, \nu^{b} \rho\right]\right) \rtimes \tau_{j},
$$


or, equivalently, such that $\mu^{*}\left(\sigma_{i}\right)$ contains the irreducible constituent

$$
\delta\left(\left[\nu \rho, \nu^{a} \rho\right]\right) \times \delta\left(\left[\nu \rho, \nu^{b} \rho\right]\right) \otimes \tau_{j} .
$$

For $b-a \in\{1,2\}$, it follows from [5, Theorem 3.1, Theorem 3.2] and [6] that the Aubert duals of both representations $\sigma_{1}$ and $\sigma_{2}$ are unitarizable.

We also note that it follows from [25] that if $\sigma$ is a discrete series subquotient of the principal series, considered groups are split, and $\operatorname{char} F=0$, then the Aubert dual of $\sigma$ is unitarizable.

Proposition 2.5. Suppose that $\alpha=\frac{1}{2}$ and let $a, b$ denote positive halfintegers such that $a<b$. We denote two irreducible square-integrable subrepresentations of the induced representation

$$
\delta\left(\left[\nu^{-a} \rho, \nu^{b} \rho\right]\right) \rtimes S\left(-\frac{1}{2}\right)
$$

by $\sigma_{1}$ and $\sigma_{2}$. Note that $S\left(-\frac{1}{2}\right) \cong \sigma_{\text {cusp. }}$. There is a unique $i \in\{1,2\}$ such that $\mu^{*}\left(\sigma_{i}\right)$ contains the irreducible constituent

$$
\delta\left(\left[\nu^{\frac{1}{2}} \rho, \nu^{a} \rho\right]\right) \times \delta\left(\left[\nu^{\frac{1}{2}} \rho, \nu^{b} \rho\right]\right) \otimes \sigma_{\text {cusp }} .
$$

Aubert duals of representations described in Theorem 2.4 and Proposition 2.5 are determined in Section 5 .

\section{Some teCHnicAl RESUlts}

In this section we state and prove several technical results which are useful for our determination of the Aubert duals of discrete series. We note that the following lemma follows from [29], but we provide an alternative proof, entirely based on the Jacquet module calculations.

LEMMA 3.1. Let $\rho$ denote an irreducible cuspidal representation of some $G L\left(n_{\rho}, F\right)$, let a be a real number and let $s$ denote a non-negative integer. For $k \in\{0,1, \ldots, s\}$, the induced representation

$$
L\left(\nu^{a} \rho, \nu^{a+1} \rho, \ldots, \nu^{a+s} \rho\right) \times \nu^{a+k} \rho
$$

is irreducible.

Proof. It follows from [29, Proposition 3.4] that if $m^{*}\left(L\left(\nu^{a} \rho, \nu^{a+1} \rho, \ldots\right.\right.$, $\left.\left.\nu^{a+s} \rho\right)\right) \geq \nu^{x} \rho \otimes \pi^{\prime}$, for some irreducible representation $\pi^{\prime}$, then $x=a$ and $\pi^{\prime} \cong L\left(\nu^{a+1} \rho, \ldots, \nu^{a+s} \rho\right)$.

Let $\pi$ denote an irreducible subquotient of $L\left(\nu^{a} \rho, \nu^{a+1} \rho, \ldots, \nu^{a+s} \rho\right) \times$ $\nu^{a+k} \rho$, and write $\pi \cong L\left(\delta_{1}, \delta_{2}, \ldots, \delta_{l}\right)$. Note that $e\left(\delta\left(\left[\nu^{x} \rho, \nu^{y} \rho\right]\right)\right) \leq e\left(\delta\left(\left[\nu^{x^{\prime}} \rho\right.\right.\right.$, $\left.\left.\left.\nu^{y^{\prime}} \rho\right]\right)\right)$ and $x^{\prime} \leq x$ imply $y \leq y^{\prime}$ and, consequently,

$$
\delta\left(\left[\nu^{x} \rho, \nu^{y} \rho\right]\right) \times \delta\left(\left[\nu^{x^{\prime}} \rho, \nu^{y^{\prime}} \rho\right]\right) \cong \delta\left(\left[\nu^{x^{\prime}} \rho, \nu^{y^{\prime}} \rho\right]\right) \times \delta\left(\left[\nu^{x} \rho, \nu^{y} \rho\right]\right) .
$$

Thus, there is a permutation $i_{1}, i_{2}, \ldots, i_{l}$ of $1,2, \ldots, l$ such that $\delta_{1} \times \delta_{2} \times$ $\cdots \times \delta_{l} \cong \delta_{i_{1}} \times \delta_{i_{2}} \times \cdots \times \delta_{i_{l}}$ and, if we write $\delta_{i_{j}}=\delta\left(\left[\nu^{x_{j}} \rho, \nu^{y_{j}} \rho\right]\right)$, such that $x_{i} \leq x_{i+1}$ for $i=1,2, \ldots, l-1$. 
Let us determine $\delta_{i_{1}}, \delta_{i_{2}}, \ldots, \delta_{i_{l}}$. From the cuspidal support of $\pi$ we obtain that $x_{1}=a$. Note that $m^{*}(\pi) \geq \nu^{y_{1}} \rho \otimes \pi^{\prime}$ for some irreducible representation $\pi^{\prime}$. Since $\nu^{y_{1}} \rho \otimes \pi^{\prime}$ is also contained in $m^{*}\left(L\left(\nu^{a} \rho, \nu^{a+1} \rho, \ldots, \nu^{a+s} \rho\right) \times \nu^{a+k} \rho\right)$, using multiplicativity of $m^{*}$ and [11], we get that $y_{1} \in\{a, a+k\}$. Let us first assume $y_{1}=a+k$ and $k \neq 0$. Obviously, in this case $\pi^{\prime} \cong$ $L\left(\nu^{a} \rho, \nu^{a+1} \rho, \ldots, \nu^{a+s} \rho\right)$. We consider two possibilities:

1. $k \geq 2$ : Since $\pi$ is a subrepresentation of $\delta_{i_{1}} \times \delta_{i_{2}} \times \cdots \times \delta_{i_{l}}$, it is also a subrepresentation of $\nu^{y_{1}} \rho \times \nu^{y_{1}-1} \rho \times \delta\left(\left[\nu^{x_{1}} \rho, \nu^{y_{1}-2} \rho\right]\right) \times \delta_{i_{2}} \times \cdots \times \delta_{i_{l}}$. Using Frobenius reciprocity, we obtain that the Jacquet module of $\pi$ with respect to an appropriate parabolic subgroup contains $\nu^{a+k} \rho \otimes$ $\nu^{a+k-1} \rho \otimes \pi^{\prime \prime}$, for some irreducible $\pi^{\prime \prime}$. Thus, $m^{*}\left(L\left(\nu^{a} \rho, \nu^{a+1} \rho, \ldots\right.\right.$, $\left.\left.\nu^{a+s} \rho\right)\right) \geq \nu^{a+k-1} \rho \otimes \pi^{\prime \prime}$, a contradiction.

2. $k=1$ : In this case, $\delta_{i_{1}}=\delta\left(\left[\nu^{a} \rho, \nu^{a+1} \rho\right]\right)$. Frobenius reciprocity implies that the Jacquet module of $\pi$ with respect to an appropriate parabolic subgroup contains $\delta_{i_{1}} \otimes \delta_{i_{2}} \otimes \cdots \otimes \delta_{i_{l}}$ and, using transitivity of Jacquet modules, we deduce that there is some irreducible representation $\pi_{1}$ such that $m^{*}(\pi) \geq \delta_{i_{1}} \otimes \pi_{1}$ and the Jacquet module of $\pi_{1}$ with respect to an appropriate parabolic subgroup contains $\delta_{i_{2}} \otimes \cdots \otimes \delta_{i_{l}}$. It is easy to see that $\pi_{1} \cong L\left(\nu^{a+1} \rho, \ldots, \nu^{a+s} \rho\right)$, implying $\delta_{i_{2}}=\nu^{a+1} \rho$. Now we have the following embeddings and isomorphism:

$$
\begin{aligned}
\pi & \hookrightarrow \delta_{i_{1}} \times \nu^{a+1} \rho \times \delta_{i_{3}} \times \cdots \times \delta_{i_{l}} \\
& \cong \nu^{a+1} \rho \times \delta_{i_{1}} \times \delta_{i_{3}} \times \cdots \times \delta_{i_{l}} \\
& \hookrightarrow \nu^{a+1} \rho \times \nu^{a+1} \rho \times \nu^{a} \rho \times \delta_{i_{3}} \times \cdots \times \delta_{i_{l}} .
\end{aligned}
$$

By $\left[22\right.$, Lemma 3.2], there is an irreducible representation $\pi_{2}$ such that $\pi \hookrightarrow \nu^{a+1} \rho \times \nu^{a+1} \rho \times \pi_{2}$ and $m^{*}(\pi) \geq \nu^{a+1} \rho \times \nu^{a+1} \rho \otimes \pi_{2}$. It follows that $m^{*}\left(L\left(\nu^{a} \rho, \nu^{a+1} \rho, \ldots, \nu^{a+s} \rho\right)\right)$ contains an irreducible constituent of the form $\nu^{a+1} \rho \otimes \pi_{2}^{\prime}$, a contradiction.

Thus, $y_{1}=a$ and $\delta_{i_{1}}=\nu^{a} \rho$. There is some irreducible representation $\pi_{1}$ such that $m^{*}(\pi) \geq \nu^{a} \rho \otimes \pi_{1}$ and the Jacquet module of $\pi_{1}$ with respect to an appropriate parabolic subgroup contains $\delta_{i_{2}} \otimes \delta_{i_{3}} \otimes \cdots \otimes \delta_{i_{l}}$. Let us first consider the case $k=0$. Again, there are two possibilities:

1. $\pi_{1} \cong L\left(\nu^{a} \rho, \nu^{a+1} \rho, \ldots, \nu^{a+s} \rho\right)$ : Description of the Jacquet modules of $L\left(\nu^{a} \rho, \nu^{a+1} \rho, \ldots, \nu^{a+s} \rho\right)$ directly implies that $l=s+2$ and $\delta_{i_{j}}=$ $\nu^{a+j-2} \rho$ for $j=2,3, \ldots, s+2$.

2. $\pi_{1} \leq L\left(\nu^{a+1} \rho, \nu^{a+2} \rho, \ldots, \nu^{a+s} \rho\right) \times \nu^{a} \rho$ : In the same way as before we conclude that $x_{2}=a$ and $y_{2} \in\{a, a+1\}$. If $y_{2}=a+1$, then an embed$\operatorname{ding} \delta_{i_{1}} \times \delta_{i_{2}} \cong \nu^{a} \rho \times \delta\left(\left[\nu^{a} \rho, \nu^{a+1} \rho\right]\right) \hookrightarrow \nu^{a+1} \rho \times \nu^{a} \rho \times \nu^{a} \rho$, Frobenius reciprocity and transitivity of Jacquet modules imply that $m^{*}(\pi) \geq$ $\nu^{a+1} \rho \otimes \pi^{\prime}$, for some irreducible $\pi^{\prime}$, which is impossible. Thus, $y_{2}=a$, $m^{*}(\pi) \geq \nu^{a} \rho \times \nu^{a} \rho \otimes L\left(\nu^{a+1} \rho, \nu^{a+2} \rho, \ldots, \nu^{a+s} \rho\right)$ and $\delta_{i_{3}} \otimes \delta_{i_{4}} \otimes \cdots \otimes \delta_{i_{l}}$ is contained in the Jacquet module of $L\left(\nu^{a+1} \rho, \nu^{a+2} \rho, \ldots, \nu^{a+s} \rho\right)$ with 
respect to an appropriate parabolic subgroup. This again implies $l=s+2$ and $\delta_{i_{j}}=\nu^{a+j-2} \rho$, for $j=3,4, \ldots, s+2$.

If $k>0$, an inductive application of the previous procedure gives $\delta_{i_{j}}=$ $\nu^{a+j-1} \rho$ for $j=1,2, \ldots, k-1$, and

$$
L\left(\delta_{i_{k}}, \delta_{i_{k}+1}, \ldots, \delta_{i_{l}}\right) \leq L\left(\nu^{a+k-1} \rho, \nu^{a+k} \rho, \ldots, \nu^{a+s} \rho\right) \times \nu^{a+k} \rho .
$$

Obviously, $x_{k}=a+k-1$ and in the same way as before we conclude that $y_{k} \in\{a+k-1, a+k\}$.

If $y_{k}=a+k$, it follows that $\delta_{i_{k+1}} \times \delta_{i_{k+2}} \times \cdots \times \delta_{i_{l}}$ is contained in $L\left(\nu^{a+k} \rho, \ldots, \nu^{a+s} \rho\right)$. Thus, $\delta_{i_{k+1}}=\nu^{a+k} \rho$, which implies that $\delta_{i_{k}} \times \delta_{i_{k+1}} \times$ $\cdots \times \delta_{i_{l}}$ is a subrepresentation of $\nu^{a+k} \rho \times \nu^{a+k} \rho \times \nu^{a+k-1} \rho \times \delta_{i_{k+2}} \times \cdots \times \delta_{i_{l}}$. Consequently, $m^{*}\left(\delta_{i_{k}} \times \delta_{i_{k+1}} \times \cdots \times \delta_{i_{l}}\right)$ contains an irreducible constituent of the form $\nu^{a+k} \rho \times \nu^{a+k} \rho \otimes \pi^{\prime}$, but this is impossible since $m^{*}\left(L\left(\nu^{a+k-1} \rho, \nu^{a+k} \rho\right.\right.$, $\left.\ldots, \nu^{a+s} \rho\right) \times \nu^{a+k} \rho$ ) does not contain such an irreducible constituent. It follows that $y_{k}=a+k-1$ and

$$
L\left(\delta_{i_{k+1}}, \delta_{i_{k+2}}, \ldots, \delta_{i_{l}}\right) \leq L\left(\nu^{a+k} \rho, \nu^{a+k+1} \rho, \ldots, \nu^{a+s} \rho\right) \times \nu^{a+k} \rho .
$$

We see at once that $x_{k+1}=x_{k+2}=a+k$. This also gives $\delta_{i_{k+1}} \times$ $\delta_{i_{k+2}} \cong \delta_{i_{k+2}} \times \delta_{i_{k+1}}$, so for $j=1,2$ there is an irreducible representation $\pi_{j}$ such that $m^{*}\left(\delta_{i_{k+1}} \times \delta_{i_{k+2}} \times \cdots \times \delta_{i_{l}}\right) \geq \nu^{y_{k+j}} \rho \otimes \pi_{j}$. Since $m^{*}\left(L\left(\nu^{a+k} \rho, \nu^{a+k+1} \rho, \ldots, \nu^{a+s} \rho\right) \times \nu^{a+k} \rho\right) \geq \nu^{y} \rho \otimes \pi^{\prime}$, for some irreducible $\pi^{\prime}$, implies $y=a+k$, we obtain that $\delta_{i_{k+1}}=\delta_{i_{k+2}}=\nu^{a+k} \rho$. Now, in the same way as before, we conclude that $l=s+2$ and $\delta_{i_{j}}=\nu^{a+j-2} \rho$, for $j=k+3, k+4, \ldots, l$.

Consequently, every irreducible subquotient of $L\left(\nu^{a} \rho, \nu^{a+1} \rho, \ldots, \nu^{a+s} \rho\right) \times$ $\nu^{a+k} \rho$ is isomorphic to

$$
L\left(\nu^{a} \rho, \ldots, \nu^{a+k-1} \rho, \nu^{a+k} \rho, \nu^{a+k} \rho, \nu^{a+k+1} \rho, \ldots, \nu^{a+s} \rho\right) .
$$

From properties of the Langlands classification follows that the representation (3.1) is contained with multiplicity one in $L\left(\nu^{a} \rho, \nu^{a+1} \rho, \ldots, \nu^{a+s} \rho\right) \times \nu^{a+k} \rho$. Consequently, the induced representation $L\left(\nu^{a} \rho, \nu^{a+1} \rho, \ldots, \nu^{a+s} \rho\right) \times \nu^{a+k} \rho$ is irreducible.

Let $\sigma_{\text {cusp }} \in \operatorname{Irr}\left(G_{n^{\prime}}\right)$ denote a cuspidal representation and let $\rho$ denote an irreducible selfcontragredient cuspidal representation of $G L\left(n_{\rho}, F\right)$. Let $\alpha \geq 0$ denote the unique non-negative real number such that the induced representation $\nu^{\alpha} \rho \rtimes \sigma_{\text {cusp }}$ reduces, and let $k=\lceil\alpha\rceil$.

LEMMA 3.2. Suppose that $\alpha>0$ and that $\sigma$ is a subrepresentation of $\delta\left(\left[\nu^{-a_{k+1}} \rho, \nu^{a_{k+2}} \rho\right]\right) \rtimes S P\left(a_{1}, a_{2}, \ldots, a_{k}\right)$, for $-1<a_{1}<a_{2}<\cdots<a_{k}<$ $a_{k+1}<a_{k+2}$ such that $\alpha-a_{i}$ is an integer for $i=1,2, \ldots, k+2$, and that $\sigma$ is not a subrepresentation of $\delta\left(\left[\nu^{-a_{k}} \rho, \nu^{a_{k+1}} \rho\right]\right) \rtimes S P\left(a_{1}, a_{2}, \ldots, a_{k-1}, a_{k+2}\right)$. Let $\tau$ denote an irreducible tempered subrepresentation of $\delta\left(\left[\nu^{-a_{k+1}} \rho, \nu^{a_{k+1}} \rho\right]\right) \rtimes$ 
$S P\left(a_{1}, a_{2}, \ldots, a_{k}\right)$ such that $\sigma \hookrightarrow \delta\left(\left[\nu^{a_{k+1}+1} \rho, \nu^{a_{k+2}} \rho\right]\right) \rtimes \tau$. Then $\mu^{*}(\tau)$ does not contain

$\delta\left(\left[\nu^{a_{k}+1} \rho, \nu^{a_{k+1}} \rho\right]\right) \times \delta\left(\left[\nu^{a_{k}+1} \rho, \nu^{a_{k+1}} \rho\right]\right) \otimes \delta\left(\left[\nu^{-a_{k}} \rho, \nu^{a_{k}} \rho\right]\right) \rtimes S P\left(a_{1}, a_{2}, \ldots, a_{k}\right)$

or, equivalently, the Jacquet module of $\tau$ with respect to an appropriate parabolic subgroup does not contain an irreducible constituent of the form

$$
\nu^{a_{k+1}} \rho \otimes \nu^{a_{k+1}} \rho \otimes \nu^{a_{k+1}-1} \rho \otimes \nu^{a_{k+1}-1} \rho \otimes \cdots \otimes \nu^{a_{k}+1} \rho \otimes \nu^{a_{k}+1} \rho \otimes \sigma_{1},
$$

for some irreducible representation $\sigma_{1}$.

Proof. Since $\sigma$ is not a subrepresentation of

$$
\delta\left(\left[\nu^{-a_{k}} \rho, \nu^{a_{k+1}} \rho\right]\right) \rtimes S P\left(a_{1}, a_{2}, \ldots, a_{k-1}, a_{k+2}\right),
$$

by [28, Proposition 7.2], $\mu^{*}(\sigma)$ does not contain an irreducible constituent of the form $\delta\left(\left[\nu^{a_{k}+1} \rho, \nu^{a_{k+1}} \rho\right]\right) \otimes \sigma^{\prime}$. Also, by [28, Lemma 4.1], there is a unique irreducible tempered subrepresentation of $\delta\left(\left[\nu^{-a_{k+1}} \rho, \nu^{a_{k+1}} \rho\right]\right) \rtimes$ $S P\left(a_{1}, a_{2}, \ldots, a_{k}\right)$ which contains

$$
\delta\left(\left[\nu^{a_{k}+1} \rho, \nu^{a_{k+1}} \rho\right]\right) \times \delta\left(\left[\nu^{a_{k}+1} \rho, \nu^{a_{k+1}} \rho\right]\right) \otimes \delta\left(\left[\nu^{-a_{k}} \rho, \nu^{a_{k}} \rho\right]\right) \rtimes S P\left(a_{1}, a_{2}, \ldots, a_{k}\right)
$$

in the Jacquet module with respect to an appropriate parabolic subgroup.

Suppose that $\mu^{*}(\tau)$ contains the irreducible constituent (3.2). Since $\sigma$ is a subrepresentation of $\delta\left(\left[\nu^{a_{k+1}+1} \rho, \nu^{a_{k+2}} \rho\right]\right) \rtimes \tau$, Frobenius reciprocity and transitivity of Jacquet modules imply that the Jacquet module of $\sigma$ with respect to an appropriate parabolic subgroup contains

$$
\begin{aligned}
& \delta\left(\left[\nu^{a_{k+1}+1} \rho, \nu^{a_{k+2}} \rho\right]\right) \otimes \delta\left(\left[\nu^{a_{k}+1} \rho, \nu^{a_{k+1}} \rho\right]\right) \times \delta\left(\left[\nu^{a_{k}+1} \rho, \nu^{a_{k+1}} \rho\right]\right) \otimes \\
& \otimes \delta\left(\left[\nu^{-a_{k}} \rho, \nu^{a_{k}} \rho\right]\right) \rtimes S P\left(a_{1}, a_{2}, \ldots, a_{k}\right) .
\end{aligned}
$$

Using transitivity of Jacquet modules again, we assert that $\mu^{*}(\sigma) \geq \delta \otimes$ $\delta\left(\left[\nu^{-a_{k}} \rho, \nu^{a_{k}} \rho\right]\right) \rtimes S P\left(a_{1}, a_{2}, \ldots, a_{k}\right)$, for some irreducible representation $\delta$ such that

$$
m^{*}(\delta) \geq \delta\left(\left[\nu^{a_{k+1}+1} \rho, \nu^{a_{k+2}} \rho\right]\right) \otimes \delta\left(\left[\nu^{a_{k}+1} \rho, \nu^{a_{k+1}} \rho\right]\right) \times \delta\left(\left[\nu^{a_{k}+1} \rho, \nu^{a_{k+1}} \rho\right]\right) .
$$

Since $\mu^{*}(\sigma) \leq \mu^{*}\left(\delta\left(\left[\nu^{-a_{k+1}} \rho, \nu^{a_{k+2}} \rho\right]\right) \rtimes S P\left(a_{1}, a_{2}, \ldots, a_{k}\right)\right)$, using Lemma 2.2 and a description of the cuspidal support of $\delta$, we conclude that

$$
\delta \leq \delta\left(\left[\nu^{a_{k}+1} \rho, \nu^{a_{k+2}} \rho\right]\right) \times \delta\left(\left[\nu^{a_{k}+1} \rho, \nu^{a_{k+1}} \rho\right]\right) .
$$

Since the induced representation $\delta\left(\left[\nu^{a_{k}+1} \rho, \nu^{a_{k+2}} \rho\right]\right) \times \delta\left(\left[\nu^{a_{k}+1} \rho, \nu^{a_{k+1}} \rho\right]\right)$ is irreducible, this implies that $\mu^{*}(\sigma) \geq \delta\left(\left[\nu^{a_{k}+1} \rho, \nu^{a_{k+1}} \rho\right]\right) \otimes \sigma^{\prime}$, for some irreducible $\sigma^{\prime}$, a contradiction.

It remains to prove that $\mu^{*}(\tau)$ contains the irreducible constituent (3.2) if and only if the Jacquet module of $\tau$ with respect to an appropriate parabolic subgroup contains an irreducible constituent of the form

$$
\nu^{a_{k+1}} \rho \otimes \nu^{a_{k+1}} \rho \otimes \nu^{a_{k+1}-1} \rho \otimes \nu^{a_{k+1}-1} \rho \otimes \cdots \otimes \nu^{a_{k}+1} \rho \otimes \nu^{a_{k}+1} \rho \otimes \sigma_{1} .
$$


Let us first assume that the Jacquet module of $\tau$ with respect to an appropriate parabolic subgroup contains an irreducible constituent of the form

$$
\nu^{a_{k+1}} \rho \otimes \nu^{a_{k+1}} \rho \otimes \nu^{a_{k+1}-1} \rho \otimes \nu^{a_{k+1}-1} \rho \otimes \cdots \otimes \nu^{a_{k}+1} \rho \otimes \nu^{a_{k}+1} \rho \otimes \sigma_{1} .
$$

Transitivity of Jacquet modules implies that there is some irreducible constituent $\delta^{\prime} \otimes \sigma_{1}$ of $\mu^{*}(\tau)$ such that $\nu^{a_{k+1}} \rho \otimes \nu^{a_{k+1}} \rho \otimes \nu^{a_{k+1}-1} \rho \otimes \nu^{a_{k+1}-1} \rho \otimes$ $\cdots \otimes \nu^{a_{k}+1} \rho \otimes \nu^{a_{k}+1} \rho$ is contained in the Jacquet module of $\delta^{\prime}$ with respect to an appropriate parabolic subgroup. Now, calculating

$$
\mu^{*}\left(\delta\left(\left[\nu^{-a_{k+1}} \rho, \nu^{a_{k+1}} \rho\right]\right) \rtimes S P\left(a_{1}, a_{2}, \ldots, a_{k}\right)\right),
$$

using Lemma 2.2, we deduce that there are $-a_{k+1}-1 \leq i \leq j \leq a_{k+1}$ and an irreducible constituent $\delta^{\prime \prime} \otimes \sigma_{1}^{\prime}$ of $\mu^{*}\left(S P\left(a_{1}, a_{2}, \ldots, a_{k}\right)\right)$ such that $\delta^{\prime} \leq \delta\left(\left[\nu^{-i} \rho, \nu^{a_{k+1}} \rho\right]\right) \times \delta\left(\left[\nu^{j+1} \rho, \nu^{a_{k+1}} \rho\right]\right) \times \delta^{\prime \prime}$ and $\sigma_{1} \leq \delta\left(\left[\nu^{i+1} \rho, \nu^{j} \rho\right]\right) \rtimes \sigma_{1}^{\prime}$. From the cuspidal support of $\delta^{\prime}$, we deduce that $\sigma_{1}^{\prime} \cong S P\left(a_{1}, a_{2}, \ldots, a_{k}\right)$, $i=-a_{k}-1$, and $j=a_{k}$. Consequently, $\delta^{\prime} \cong \delta\left(\left[\nu^{a_{k}+1} \rho, \nu^{a_{k+1}} \rho\right]\right) \times$ $\delta\left(\left[\nu^{a_{k}+1} \rho, \nu^{a_{k+1}} \rho\right]\right)$ and $\sigma_{1} \cong \delta\left(\left[\nu^{-a_{k}} \rho, \nu^{a_{k}} \rho\right]\right) \rtimes S P\left(a_{1}, a_{2}, \ldots, a_{k}\right)$, so $\mu^{*}(\tau)$ contains the irreducible constituent (3.2).

Let us now suppose that $\mu^{*}(\tau)$ contains the irreducible constituent (3.2). Since the induced representation $\delta\left(\left[\nu^{a_{k}+1} \rho, \nu^{a_{k+1}} \rho\right]\right) \times \delta\left(\left[\nu^{a_{k}+1} \rho, \nu^{a_{k+1}} \rho\right]\right)$ is irreducible, it follows that the Jacquet module of $\tau$ with respect to an appropriate parabolic subgroup contains

$$
\begin{array}{r}
\nu^{a_{k+1}} \rho \otimes \nu^{a_{k+1}} \rho \otimes \nu^{a_{k+1}-1} \rho \otimes \nu^{a_{k+1}-1} \rho \otimes \cdots \otimes \nu^{a_{k}+1} \rho \otimes \nu^{a_{k}+1} \rho \otimes \\
\otimes \delta\left(\left[\nu^{-a_{k}} \rho, \nu^{a_{k}} \rho\right]\right) \rtimes S P\left(a_{1}, a_{2}, \ldots, a_{k}\right)
\end{array}
$$

and lemma is proved.

Proof of the following lemma follows directly from [14, Theorem 4.6] and [16, Section 4].

Lemma 3.3. Suppose that $\alpha>0$ and let $\sigma$ denote an irreducible subrepresentation of the induced representation

$$
\delta\left(\left[\nu^{-a_{m}} \rho, \nu^{a_{m+1}} \rho\right]\right) \rtimes S P\left(a_{1}, \ldots, a_{m-1}, a_{m+2}, \ldots, a_{k+2}\right),
$$

where $-1<a_{1}<a_{2}<\cdots<a_{k+2}$ such that $\alpha-a_{i}$ is an integer for $i=$ $1,2, \ldots, k+2$. Let $i_{\max }$ denote the maximal $i$ such that the Jacquet module of $\sigma$ with respect to an appropriate parabolic subgroup contains an irreducible constituent of the form $\nu^{i} \rho \otimes \nu^{i+1} \rho \otimes \cdots \otimes \nu^{a_{k+2}} \rho \otimes \sigma^{\prime}$. Also, let l denote the maximal $i \in\{2,3, \ldots, k+2\}$ such that $a_{i} \geq a_{i-1}+2$, or let $l=0$, if such $i$ does not exist. If $l>m+1$, then $i_{\max }=a_{l}$, otherwise $i_{\max }=a_{m+1}$. 
Furthermore, if $l>m+1$ we have

$$
\begin{aligned}
\delta\left(\left[\nu^{-a_{k+2}} \rho, \nu^{-i_{\max }} \rho\right]\right) \cong\left(\prod_{i=1}^{k-l+2} \prod_{j=-a_{k-i+3}}^{-a_{k-i+2}-2} \delta(\right. & {\left.\left.\left[\nu^{j-i+1} \rho, \nu^{j} \rho\right]\right)\right) \times } \\
& \times \delta\left(\left[\nu^{-a_{l}-(k-l+3)+1} \rho, \nu^{-a_{l}} \rho\right]\right),
\end{aligned}
$$

and otherwise we have

$$
\begin{aligned}
\delta\left(\left[\nu^{-a_{k+2}} \rho, \nu^{-i_{\max }} \rho\right]\right) \cong\left(\prod_{i=1}^{k-m+1}\right. & \left.\prod_{j=-a_{k-i+3}}^{-a_{k-i+2}-2} \delta\left(\left[\nu^{j-i+1} \rho, \nu^{j} \rho\right]\right)\right) \times \\
& \times \delta\left(\left[\nu^{-a_{m+1}-(k-m+2)+1} \rho, \nu^{-a_{m+1}} \rho\right]\right) .
\end{aligned}
$$

LEMma 3.4. Suppose that $\sigma \in \operatorname{Irr}\left(G_{n}\right)$ is a subrepresentation of an induced representation of the form $\nu^{x} \rho \times \nu^{x-1} \rho \times \cdots \times \nu^{x-l} \rho \rtimes \sigma^{\prime}$, for some irreducible representations $\rho$ and $\sigma^{\prime}$, and that $\mu^{*}(\sigma)$ does not contain an irreducible constituent of the form $\nu^{y} \rho \otimes \sigma^{\prime \prime}$ for $y \in\{x-l, x-l+1, \ldots, x-1\}$. Then $\sigma$ is a subrepresentation of $\delta\left(\left[\nu^{x-l} \rho, \nu^{x} \rho\right]\right) \rtimes \sigma^{\prime}$.

Proof. Condition regarding the Jacquet modules of $\sigma$ implies that $\sigma$ is contained in the kernel of an intertwining operator

$$
\nu^{x} \rho \times \nu^{x-1} \rho \times \cdots \times \nu^{x-l} \rho \rtimes \sigma^{\prime} \rightarrow \nu^{x-1} \rho \times \nu^{x} \rho \times \cdots \times \nu^{x-l} \rho \rtimes \sigma^{\prime} .
$$

Thus, $\sigma$ is a subrepresentation of $\delta\left(\left[\nu^{x-1} \rho, \nu^{x} \rho\right]\right) \times \nu^{x-2} \rho \times \cdots \times \nu^{x-l} \rho \rtimes \sigma^{\prime}$. In the same way we conclude that $\sigma$ is contained in the kernel of an intertwining operator

$$
\begin{aligned}
\delta\left(\left[\nu^{x-1} \rho, \nu^{x} \rho\right]\right) \times \nu^{x-2} \rho \times \cdots \times \nu^{x-l} \rho \rtimes \sigma^{\prime} \rightarrow & \\
\nu^{x-2} \rho & \times \delta\left(\left[\nu^{x-1} \rho, \nu^{x} \rho\right]\right) \times \nu^{x-3} \rho \times \cdots \times \nu^{x-l} \rho \rtimes \sigma^{\prime} .
\end{aligned}
$$

Consequently, $\sigma$ is a subrepresentation of $\delta\left(\left[\nu^{x-2} \rho, \nu^{x} \rho\right]\right) \times \nu^{x-3} \rho \times \cdots \times \nu^{x-l} \rho \rtimes$ $\sigma^{\prime}$. A repeated application of this procedure finishes the proof.

\section{Aubert duals of Discrete Series}

In this section we begin with a determination of the Aubert duals of certain discrete series representations.

In the rest of the paper we fix an irreducible cuspidal representation $\sigma_{\text {cusp }} \in \operatorname{Irr}\left(G_{n_{1}}\right)$ and an irreducible selfcontragredient cuspidal representation $\rho$ of some $G L\left(n_{\rho}, F\right)$. Let $\alpha \geq 0$ denote the unique non-negative real number such that the induced representation $\nu^{\alpha} \rho \rtimes \sigma_{\text {cusp }}$ reduces, and let $k=\lceil\alpha\rceil$.

In this section we assume $\alpha>0$. Also, if $\alpha=\frac{1}{2}$, in this section we only consider strongly positive representations of the form $S P(a)$ where $a>-\frac{1}{2}$. Remaining cases will be considered in the next section. 
As we have already mentioned, we consider only discrete series whose cuspidal support consists of the twist of $\rho$ and of $\sigma_{\text {cusp }}$.

Let us first introduce the concept of the $(\sigma, \widehat{\sigma})$-triple which enables us to obtain an explicit description of the Aubert duals using an inductive procedure.

Definition 4.1. Let $\sigma \in \operatorname{Irr}\left(G_{n}\right)$ denote a discrete series representation, and let $\widehat{\sigma}$ denote its Aubert dual. Let $\pi, \pi^{\prime}$ denote irreducible admissible representations of $G_{n^{\prime}}$ for some $n^{\prime}$. Let $l$ denote a non-negative integer and let $T$ be a (possibly empty) set $\left\{\left(x_{1}, y_{1}\right),\left(x_{2}, y_{2}\right), \ldots,\left(x_{l}, y_{l}\right)\right\}$ where $0<y_{i} \leq x_{i}$, $\alpha-x_{i} \in \mathbb{Z}$ and $\alpha-y_{i} \in \mathbb{Z}$, for $i=1,2, \ldots, l$. We say that an ordered triple $\left(T, \pi, \pi^{\prime}\right)$ is a $(\sigma, \widehat{\sigma})$-triple if the following holds:

(a) for an appropriate parabolic subgroup $P$ of $G_{n}$ with the Levi subgroup $M$, we have

$r_{M}(\sigma) \geq \nu^{y_{1}} \rho \otimes \nu^{y_{1}+1} \rho \otimes \cdots \otimes \nu^{x_{1}} \rho \otimes \cdots \otimes \nu^{y_{l}} \rho \otimes \nu^{y_{l}+1} \rho \otimes \cdots \otimes \nu^{x_{l}} \rho \otimes \pi$,

and, if

$r_{M}(\sigma) \geq \nu^{y_{1}} \rho \otimes \nu^{y_{1}+1} \rho \otimes \cdots \otimes \nu^{x_{1}} \rho \otimes \cdots \otimes \nu^{y_{l}} \rho \otimes \nu^{y_{l}+1} \rho \otimes \cdots \otimes \nu^{x_{l}} \rho \otimes \pi^{\prime \prime}$,

for some irreducible representation $\pi^{\prime \prime}$, then $\pi^{\prime \prime} \cong \pi$.

(b) $\widehat{\sigma} \hookrightarrow \delta_{1} \times \delta_{2} \times \cdots \times \delta_{l} \rtimes \pi^{\prime}$, where $\delta_{i}=\delta\left(\left[\nu^{-x_{i}} \rho, \nu^{-y_{i}} \rho\right]\right)$, for $i=1,2, \ldots, l$, and

(b1) if $e\left(\delta_{i}\right)>e\left(\delta_{j}\right)$ for $i<j$, then $\delta_{i} \times \delta_{j} \cong \delta_{j} \times \delta_{i}$,

(b2) if $\pi^{\prime}=L\left(\delta_{l+1}, \delta_{l+2}, \ldots, \delta_{m}, \tau\right)$ and $e\left(\delta_{i}\right)>e\left(\delta_{j}\right)$ for $i \leq l$ and $j \geq l+1$, then $\delta_{i} \times \delta_{j} \cong \delta_{j} \times \delta_{i}$,

(b3) if for some parabolic subgroup $P^{\prime}$ of $G_{n}$ having the Levi subgroup $M^{\prime}$ we have

$$
r_{M^{\prime}}(\widehat{\sigma}) \geq \nu^{-y_{1}} \rho \otimes \cdots \otimes \nu^{-x_{1}} \rho \otimes \cdots \otimes \nu^{-y_{l}} \rho \otimes \cdots \otimes \nu^{-x_{l}} \rho \otimes \pi^{\prime \prime},
$$

for an irreducible representation $\pi^{\prime \prime}$, then $\pi^{\prime \prime} \leq r_{M^{\prime \prime}}\left(\pi^{\prime}\right)$ for an appropriate parabolic subgroup $P^{\prime \prime}$ having the Levi subgroup $M^{\prime \prime}$.

We emphasize that parts $(a)$ and (b3) of the previous definition, together with the first embedding in $(b)$, allow us to determine the Aubert dual of $\sigma$ using an inductive procedure, studying the Jacquet modules of $\pi$ and using properties of the Aubert involution. Also, properties (b1) and (b2) ensure that in each step $\widehat{\sigma}$ is given as a subrepresentation of an induced representation having a unique irreducible (Langlands) subrepresentation. This follows from a simple commuting argument, and is also stated in Lemma 4.3.

Note that for a discrete series $\sigma \in \operatorname{Irr}\left(G_{n}\right),(\emptyset, \sigma, \hat{\sigma})$ is a $(\sigma, \hat{\sigma})$-triple in a trivial way.

The following elementary lemma, which is a direct consequence of the transitivity of Jacquet modules, is useful for our considerations. 
Lemma 4.2. Suppose that $\sigma \in \operatorname{Irr}\left(G_{n}\right), \pi_{1} \in \operatorname{Irr}\left(G L\left(n_{1}, F\right)\right), n_{1}<n$, and $\pi_{2} \in \operatorname{Irr}\left(G_{n-n_{1}}\right)$ are such that if $\mu^{*}(\sigma)$ contains an irreducible constituent of the form $\pi_{1} \otimes \pi^{\prime}$, then $\pi^{\prime} \cong \pi_{2}$. For irreducible representations $\pi_{3} \in$ $\operatorname{Irr}\left(G L\left(n_{3}, F\right)\right), n_{3}<n-n_{1}$, and $\pi_{4} \in \operatorname{Irr}\left(G_{n-n_{1}-n_{3}}\right)$, the following two statements are equivalent:

1. If the Jacquet module of $\sigma$ with respect to an appropriate parabolic subgroup contains an irreducible constituent $\pi_{1} \otimes \pi_{3} \otimes \pi^{\prime}$ then $\pi^{\prime} \cong \pi_{4}$.

2. If the Jacquet module of $\pi_{2}$ with respect to an appropriate parabolic subgroup contains an irreducible constituent $\pi_{3} \otimes \pi^{\prime}$ then $\pi^{\prime} \cong \pi_{4}$.

Proof of the following lemma is immediate.

Lemma 4.3. Suppose that an irreducible representation $\pi$ of $G_{n}$ is a subrepresentation of the induced representation of the form $\delta_{1} \times \delta_{2} \times \cdots \times \delta_{l} \rtimes \pi_{1}$, where $\pi_{1}$ is irreducible, $\delta_{i} \in \operatorname{Irr}\left(G L\left(n_{i}, F\right)\right)$ is an essentially square-integrable representation such that $e\left(\delta_{i}\right)<0$ for $i=1,2, \ldots, l$, and if $e\left(\delta_{i}\right)>e\left(\delta_{j}\right)$ for $i<j$, then $\delta_{i} \times \delta_{j} \cong \delta_{j} \times \delta_{i}$. Let $\pi_{1}=L\left(\delta_{l+1}, \delta_{l+2}, \ldots, \delta_{m}, \tau\right)$ and suppose that if $e\left(\delta_{i}\right)>e\left(\delta_{j}\right)$ for $i \leq l$ and $j \geq l+1$, then $\delta_{i} \times \delta_{j} \cong \delta_{j} \times \delta_{i}$. Then there is a permutation $\delta_{i_{1}}, \delta_{i_{2}}, \ldots, \delta_{i_{m}}$ of $\delta_{1}, \delta_{2}, \ldots, \delta_{m}$ such that $e\left(\delta_{i_{1}}\right) \leq e\left(\delta_{i_{2}}\right) \leq$ $\cdots \leq e\left(\delta_{i_{m}}\right)$ and

$$
\delta_{i_{1}} \times \delta_{i_{2}} \times \cdots \times \delta_{i_{m}} \cong \delta_{1} \times \delta_{2} \times \cdots \times \delta_{m} .
$$

In particular, $\pi=L\left(\delta_{i_{1}}, \delta_{i_{2}}, \ldots, \delta_{i_{m}}, \tau\right)$.

We now prove several results which will be useful in the verification of properties given in Definition 4.1.

LEMmA 4.4. Suppose that $\sigma \in \operatorname{Irr}\left(G_{n}\right)$ is a subrepresentation of an induced representation of the form

$$
\delta\left(\left[\nu^{-a} \rho, \nu^{-b_{1}} \rho\right]\right) \times \delta\left(\left[\nu^{-a} \rho, \nu^{-b_{2}} \rho\right]\right) \times \cdots \times \delta\left(\left[\nu^{-a} \rho, \nu^{-b_{r}} \rho\right]\right) \rtimes \sigma_{1},
$$

where $\rho \in \operatorname{Irr}\left(G L\left(n_{\rho}, F\right)\right)$ is self-contragredient, $a>b_{i}>0, a-b_{i} \in \mathbb{Z}$ for $i=1,2, \ldots, r$, and suppose that $\nu^{a} \rho$ does not appear in the cuspidal support of $\sigma_{1}$. If the Jacquet module of $\sigma$ with respect to an appropriate parabolic subgroup contains an irreducible constituent of the form

$\nu^{-b_{1}} \rho \otimes \cdots \otimes \nu^{-a} \rho \otimes \cdots \otimes \nu^{-b_{2}} \rho \otimes \cdots \otimes \nu^{-a} \rho \otimes \cdots \otimes \nu^{-b_{r}} \rho \otimes \cdots \otimes \nu^{-a} \rho \otimes \sigma_{2}$,

then $\sigma_{2} \cong \sigma_{1}$.

Proof. Transitivity of Jacquet modules implies that there is some irreducible constituent $\pi_{1} \otimes \sigma_{2}$ of $\mu^{*}(\sigma)$ such that $\nu^{-b_{1}} \rho \otimes \cdots \otimes \nu^{-a} \rho \otimes \cdots \otimes \nu^{-b_{2}} \rho \otimes$ $\cdots \otimes \nu^{-a} \rho \otimes \cdots \otimes \nu^{-b_{r}} \rho \otimes \cdots \otimes \nu^{-a} \rho$ is contained in the Jacquet module of $\pi_{1}$ with respect to an appropriate parabolic subgroup. We determine $\pi_{1}$ using the structural formula given in Lemma 2.2. Since $\sigma$ is a subrepresentation 
of the induced representation (4.1), there exist $-a-1 \leq x_{i} \leq y_{i} \leq-b_{i}$, for $i=1,2, \ldots, r$, and an irreducible constituent $\pi_{2} \otimes \sigma_{3}$ of $\mu^{*}\left(\sigma_{1}\right)$ such that

$$
\pi_{1} \leq \prod_{i=1}^{r}\left(\delta\left(\left[\nu^{-x_{i}} \rho, \nu^{a} \rho\right]\right) \times \delta\left(\left[\nu^{y_{i}+1} \rho, \nu^{-b_{i}} \rho\right]\right)\right) \times \pi_{2}
$$

and

$$
\sigma_{2} \leq \prod_{i=1}^{r}\left(\delta\left(\left[\nu^{x_{i}+1} \rho, \nu^{y_{i}} \rho\right]\right)\right) \rtimes \sigma_{3} .
$$

Since $\nu^{a} \rho$ does not appear in the cuspidal support of $\sigma_{1}, \nu^{-a} \rho$ does not appear in the cuspidal support of $\pi_{2}$. Furthemore, since $-x_{i}>0$, it follows that $x_{i}=y_{i}=-a-1$ for all $i=1,2, \ldots, r$. Consequently, $\sigma_{3} \cong \sigma_{1}$ and $\sigma_{2} \cong \sigma_{1}$. This finishes the proof.

In the same way as in the proof of the previous lemma, one obtains the following result, where $\left(\nu^{-c} \rho\right)^{m}$ denotes the product $\nu^{-c} \rho \times \cdots \times \nu^{-c} \rho$, where $\nu^{-c} \rho$ appears $m$ times:

Lemma 4.5. Suppose that $\sigma \in \operatorname{Irr}\left(G_{n}\right)$ is a subrepresentation of an induced representation of the form

$$
\delta\left(\left[\nu^{-a} \rho, \nu^{-b} \rho\right]\right) \times\left(\nu^{-c} \rho\right)^{m} \rtimes \sigma_{1},
$$

where $\rho \in \operatorname{Irr}\left(G L\left(n_{\rho}, F\right)\right)$ is self-contragredient, $a>b>0, a-b \in \mathbb{Z}, \nu^{a} \rho$ does not appear in the cuspidal support of $\sigma_{1}$, and $\mu^{*}\left(\sigma_{1}\right)$ does not contain an irreducible constituent of the form $\nu^{-c} \rho \otimes \sigma_{1}^{\prime}$. If the Jacquet module of $\sigma$ with respect to an appropriate parabolic subgroup contains an irreducible constituent of the form

$$
\nu^{-b} \rho \otimes \nu^{-b-1} \rho \otimes \cdots \otimes \nu^{-a} \rho \otimes \nu^{-c} \rho \otimes \cdots \otimes \nu^{-c} \rho \otimes \sigma_{2},
$$

where $\nu^{-c} \rho$ appears $m$ times, then $\sigma_{2} \cong \sigma_{1}$.

In the following lemma we prove some properties of $(\sigma, \widehat{\sigma})$-triples which are frequently used in the paper.

LemMA 4.6. Suppose that $\left(T, \pi, \pi^{\prime}\right)$ is a $(\sigma, \widehat{\sigma})$-triple and let a stand for the maximal positive $i$ such that $\nu^{i} \rho$ appears in the cuspidal support of $\pi$. Let us denote by $b$ the maximal $j$ such that the Jacquet module of $\pi$ with respect to an appropriate parabolic subgroup contains an irreducible constituent of the form $\nu^{j} \rho \otimes \nu^{j+1} \rho \otimes \cdots \otimes \nu^{a} \rho \otimes \sigma^{\prime}$. Then there is some irreducible representation $\pi_{r}^{\prime}$ such that $\pi^{\prime}$ is a subrepresentation of $\delta\left(\left[\nu^{-a} \rho, \nu^{-b} \rho\right]\right) \rtimes \pi_{r}^{\prime}$, and if $\pi_{r}^{\prime}=$ $L\left(\delta_{1}^{\prime}, \delta_{2}^{\prime}, \ldots, \delta_{m}^{\prime}, \tau\right)$ and $e\left(\delta\left(\left[\nu^{-a} \rho, \nu^{-b} \rho\right]\right)\right)>e\left(\delta_{i}^{\prime}\right)$ for some $i \in\{1,2, \ldots, m\}$, then $\delta\left(\left[\nu^{-a} \rho, \nu^{-b} \rho\right]\right) \times \delta_{i}^{\prime} \cong \delta_{i}^{\prime} \times \delta\left(\left[\nu^{-a} \rho, \nu^{-b} \rho\right]\right)$. 
Proof. Let $l=|T|$. Transitivity of Jacquet modules implies that the Jacquet module of $\sigma$ with respect to an appropriate parabolic subgroup contains an irreducible constituent of the form

$$
\begin{aligned}
& \nu^{y_{1}} \rho \otimes \nu^{y_{1}-1} \rho \otimes \cdots \otimes \nu^{x_{1}} \rho \otimes \cdots \otimes \nu^{y_{l}} \rho \otimes \nu^{y_{l}-1} \rho \otimes \cdots \otimes \nu^{x_{l}} \rho \otimes \\
& \otimes \nu^{b} \rho \otimes \nu^{b+1} \rho \otimes \cdots \otimes \nu^{a} \rho \otimes \pi_{1} .
\end{aligned}
$$

Elementary properties of the Aubert involution, given in Theorem $2.1(\mathrm{iv})$, now imply that the Jacquet module of $\widehat{\sigma}$ with respect to an appropriate parabolic subgroup contains an irreducible constituent of the form

$$
\begin{array}{r}
\nu^{-y_{1}} \rho \otimes \nu^{-y_{1}+1} \rho \otimes \cdots \otimes \nu^{-x_{1}} \rho \otimes \cdots \otimes \nu^{-y_{l}} \rho \otimes \nu^{-y_{l}+1} \rho \otimes \cdots \otimes \nu^{-x_{l}} \rho \otimes \\
\otimes \nu^{-b} \rho \otimes \nu^{-b-1} \rho \otimes \cdots \otimes \nu^{-a} \rho \otimes \pi_{1}^{\prime} .
\end{array}
$$

Now property (b3) from Definition 4.1, together with transitivity of Jacquet modules, shows that the Jacquet module of $\pi^{\prime}$ with respect to an appropriate parabolic subgroup contains an irreducible constituent of the form

$$
\nu^{-b} \rho \otimes \nu^{-b-1} \rho \otimes \cdots \otimes \nu^{-a} \rho \otimes \pi_{2}^{\prime} .
$$

From [22, Lemma 3.1], we deduce that $\pi^{\prime}$ is a subrepresentation of an induced representation of the form

$$
\nu^{-b} \rho \times \nu^{-b-1} \rho \times \cdots \times \nu^{-a} \rho \rtimes \pi_{3}^{\prime} .
$$

Suppose that $\pi^{\prime}$ is not contained in the kernel of an intertwining operator

$$
\begin{gathered}
\nu^{-b} \rho \times \nu^{-b-1} \rho \times \cdots \times \nu^{i+1} \rho \times \nu^{i} \rho \times \nu^{i-1} \rho \times \nu^{i-2} \rho \times \cdots \times \nu^{-a} \rho \rtimes \pi_{3}^{\prime} \rightarrow \\
\nu^{-b} \rho \times \nu^{-b-1} \rho \times \cdots \times \nu^{i+1} \rho \times \nu^{i-1} \rho \times \nu^{i} \rho \times \nu^{i-2} \rho \times \cdots \times \nu^{-a} \rho \rtimes \pi_{3}^{\prime},
\end{gathered}
$$

for some $i \in\{-b,-b-1, \ldots,-a+1\}$. Since $\nu^{x} \rho \times \nu^{y} \rho \cong \nu^{y} \rho \times \nu^{x} \rho$, for $x \leq y-2$, it follows that $\pi^{\prime}$ is a subrepresentation of an induced representation of the form

$$
\nu^{i-1} \rho \times \nu^{i-2} \rho \times \cdots \times \nu^{-a} \rho \rtimes \pi_{4}^{\prime} .
$$

Using transitivity of Jacquet modules again, it follows that the Jacquet module of $\widehat{\sigma}$ with respect to an appropriate parabolic subgroup contains an irreducible constituent of the form

$$
\begin{aligned}
& \nu^{-y_{1}} \rho \otimes \nu^{-y_{1}+1} \rho \otimes \cdots \otimes \nu^{-x_{1}} \rho \otimes \cdots \otimes \nu^{-y_{l}} \rho \otimes \nu^{-y_{l}+1} \rho \otimes \cdots \otimes \nu^{-x_{l}} \rho \otimes \\
& \otimes \nu^{i-1} \rho \otimes \nu^{i-2} \rho \otimes \cdots \otimes \nu^{-a} \rho \otimes \pi_{4}^{\prime},
\end{aligned}
$$

and, consequently, using the properties of the Aubert involution listed in Theorem 2.1 and the part $(a)$ of Definition 4.1, the Jacquet module of $\pi$ with respect to an appropriate parabolic subgroup contains an irreducible constituent of the form

$$
\nu^{-i+1} \rho \otimes \nu^{-i+2} \rho \otimes \cdots \otimes \nu^{a} \rho \otimes \pi_{2},
$$


for $i \leq-b$, contradicting the choice of $b$. Thus, $\pi^{\prime}$ is contained in the kernel of intertwining operator (4.2) for all $i \in\{-b,-b-1, \ldots,-a+1\}$. In the same way as in the proof of Lemma 3.4, we see that $\pi^{\prime}$ is a subrepresentation of $\delta\left(\left[\nu^{-a} \rho, \nu^{-b} \rho\right]\right) \rtimes \pi_{3}^{\prime}$.

Let us now assume that $\pi_{3}^{\prime}=L\left(\delta_{1}^{\prime}, \delta_{2}^{\prime}, \ldots, \delta_{m}^{\prime}, \tau\right)$ and $e\left(\delta\left(\left[\nu^{-a} \rho, \nu^{-b} \rho\right]\right)\right)>$ $e\left(\delta_{i}^{\prime}\right)$, for some $i \in\{1,2, \ldots, m\}$. Let us write $\delta_{i}^{\prime}=\delta\left(\left[\nu^{x} \rho, \nu^{y} \rho\right]\right)$. Obviously, $x<0$ and, from the definition of $a$ and a description of the cuspidal support of $\pi_{3}^{\prime}$, we deduce that $-a \leq x$. Since $e\left(\delta\left(\left[\nu^{-a} \rho, \nu^{-b} \rho\right]\right)\right)$ is greater than $e\left(\delta\left(\left[\nu^{x} \rho, \nu^{y} \rho\right]\right)\right)$, it follows that $y \leq-b$ and, consequently,

$$
\delta\left(\left[\nu^{-a} \rho, \nu^{-b} \rho\right]\right) \times \delta\left(\left[\nu^{x} \rho, \nu^{y} \rho\right]\right) \cong \delta\left(\left[\nu^{x} \rho, \nu^{y} \rho\right]\right) \times \delta\left(\left[\nu^{-a} \rho, \nu^{-b} \rho\right]\right) .
$$

This finishes the proof.

From the proof of Lemma 4.6, we obtain the following result.

Corollary 4.7. Suppose that $\left(T, \pi, \pi^{\prime}\right)$ is a $(\sigma, \widehat{\sigma})$-triple and that $\pi$ is a subrepresentation of an induced representation of the form

$$
\nu^{x} \rho \times \nu^{x+1} \rho \times \cdots \times \nu^{x+r} \rho \rtimes \sigma_{1},
$$

where $\sigma_{1}$ is not necessarily irreducible, and $\mu^{*}(\pi)$ does not contain an irreducible constituent of the form $\nu^{y} \rho \otimes \sigma_{2}$ for $y=x+1, \ldots, x+r$. Then there is an irreducible representation $\tau$ such that $\pi^{\prime}$ is a subrepresentation of

$$
\delta\left(\left[\nu^{-x-r} \rho, \nu^{-x} \rho\right]\right) \rtimes \tau .
$$

We now prove a sequence of lemmas which lead to a description of the Aubert duals of studied discrete series representations.

Lemma 4.8. Suppose that $\left(T, \pi, \pi^{\prime}\right)$ is a $(\sigma, \widehat{\sigma})$-triple such that $\pi$ is a subrepresentation of

$$
\delta\left(\left[\nu^{-a_{k+1}} \rho, \nu^{a_{k+2}} \rho\right]\right) \rtimes S P\left(a_{1}, a_{2}, \ldots, a_{k}\right),
$$

for $-1<a_{1}<a_{2}<\cdots<a_{k}<a_{k+1}<a_{k+2}$ such that $\alpha-a_{i}$ is an integer for $i=1,2, \ldots, k+2$. Let $l=|T|$. Let $x_{l+1}=y_{l+1}=a_{k+2}$ and let $\pi_{1}^{\prime}$ denote an irreducible representation such that $\pi^{\prime} \hookrightarrow \nu^{-a_{k+2}} \rho \rtimes \pi_{1}^{\prime}$.

(i) Suppose that $\pi$ is not a subrepresentation of

$$
\delta\left(\left[\nu^{-a_{k}} \rho, \nu^{a_{k+1}} \rho\right]\right) \rtimes S P\left(a_{1}, a_{2}, \ldots, a_{k-1}, a_{k+2}\right) .
$$

If $a_{k+2}>a_{k+1}+1$, let $\pi_{1}$ stand for an irreducible subrepresentation of

$$
\delta\left(\left[\nu^{-a_{k+1}} \rho, \nu^{a_{k+2}-1} \rho\right]\right) \rtimes S P\left(a_{1}, a_{2}, \ldots, a_{k}\right)
$$

which is not an irreducible subrepresentation of

$$
\delta\left(\left[\nu^{-a_{k}} \rho, \nu^{a_{k+1}} \rho\right]\right) \rtimes S P\left(a_{1}, a_{2}, \ldots, a_{k-1}, a_{k+2}-1\right) .
$$

If $a_{k+2}=a_{k+1}+1$, let $\pi_{1}$ stand for the unique irreducible subrepresentation of $\delta\left(\left[\nu^{-a_{k+1}} \rho, \nu^{a_{k+1}} \rho\right]\right) \rtimes S P\left(a_{1}, a_{2}, \ldots, a_{k}\right)$ which does not contain $\delta\left(\left[\nu^{a_{k}+1} \rho, \nu^{a_{k+1}} \rho\right]\right) \times \delta\left(\left[\nu^{a_{k}+1} \rho, \nu^{a_{k+1}} \rho\right]\right) \otimes \delta\left(\left[-\nu^{a_{k}} \rho, \nu^{a_{k}} \rho\right]\right) \rtimes S P\left(a_{1}, a_{2}, \ldots, a_{k}\right)$ 
in the Jacquet module with respect to an appropriate parabolic subgroup.

(ii) Suppose that $\pi$ is a subrepresentation of

$$
\delta\left(\left[\nu^{-a_{k}} \rho, \nu^{a_{k+1}} \rho\right]\right) \rtimes S P\left(a_{1}, a_{2}, \ldots, a_{k-1}, a_{k+2}\right) .
$$

If $a_{k+2}>a_{k+1}+1$, let $\pi_{1}$ stand for the unique irreducible subrepresentation of both induced representations

$$
\delta\left(\left[\nu^{-a_{k+1}} \rho, \nu^{a_{k+2}-1} \rho\right]\right) \rtimes S P\left(a_{1}, a_{2}, \ldots, a_{k}\right)
$$

and

$$
\delta\left(\left[\nu^{-a_{k}} \rho, \nu^{a_{k+1}} \rho\right]\right) \rtimes S P\left(a_{1}, a_{2}, \ldots, a_{k-1}, a_{k+2}-1\right) .
$$

If $a_{k+2}=a_{k+1}+1$, let $\pi_{1}$ stand for the unique irreducible subrepresentation of $\delta\left(\left[\nu^{-a_{k+1}} \rho, \nu^{a_{k+1}} \rho\right]\right) \rtimes S P\left(a_{1}, a_{2}, \ldots, a_{k}\right)$ which contains

$$
\begin{aligned}
& \delta\left(\left[\nu^{a_{k}+1} \rho, \nu^{a_{k+1}} \rho\right]\right) \times \delta\left(\left[\nu^{a_{k}+1} \rho, \nu^{a_{k+1}} \rho\right]\right) \otimes \\
& \quad \otimes \delta\left(\left[-\nu^{a_{k}} \rho, \nu^{a_{k}} \rho\right]\right) \rtimes S P\left(a_{1}, a_{2}, \ldots, a_{k}\right)
\end{aligned}
$$

in the Jacquet module with respect to an appropriate parabolic subgroup. Then $\left(T \cup\left\{\left(x_{l+1}, y_{l+1}\right)\right\}, \pi_{1}, \pi_{1}^{\prime}\right)$ is a $(\sigma, \widehat{\sigma})$-triple.

Proof. We only prove $(i)$, because $(i i)$ can be proved in the same way.

By [19, Section 8] and Lemma 3.2, $\mu^{*}(\pi) \geq \nu^{a_{k+2}} \rho \otimes \pi_{1}$ and if $\mu^{*}(\pi)$ contains an irreducible constituent of the form $\nu^{a_{k+2}} \rho \otimes \pi_{2}$, then $\pi_{2} \cong \pi_{1}$. Since $\left(T, \pi, \pi^{\prime}\right)$ is a $(\sigma, \widehat{\sigma})$-triple, it follows that $\left(T \cup\left\{\left(x_{l+1}, y_{l+1}\right)\right\}, \pi_{1}, \pi_{1}^{\prime}\right)$ satisfies the part $(a)$ of Definition 4.1. Lemma 4.6 shows that it also satisfies (b1) and (b2), while the property (b3) follows from Lemma 4.4. This finishes the proof.

Lemma 4.9. Suppose that $\left(T, \pi, \pi^{\prime}\right)$ is a $(\sigma, \widehat{\sigma})$-triple such that $\pi$ is a subrepresentation of the induced representations

$$
\delta\left(\left[\nu^{-a_{k+1}} \rho, \nu^{a_{k+1}} \rho\right]\right) \rtimes S P\left(a_{1}, a_{2}, \ldots, a_{k}\right),
$$

where $-1<a_{1}<a_{2}<\cdots<a_{k}<a_{k+1}$ such that $\alpha-a_{i}$ is an integer for $i=1,2, \ldots, k+1$. Let $l=|T|$. There are two possibilities to consider:

(i) Suppose that $a_{k+1}>a_{k}+1$. Let $x_{l+1}=x_{l+2}=y_{l+1}=y_{l+2}=a_{k+1}$ and let $\pi_{1}^{\prime}$ denote an irreducible representation such that $\pi^{\prime} \hookrightarrow \nu^{-a_{k+1}} \rho \times$ $\nu^{-a_{k+1}} \rho \rtimes \pi_{1}^{\prime}$.

(a) If $\pi$ is not a subrepresentation of

$$
\delta\left(\left[\nu^{-a_{k}} \rho, \nu^{a_{k+1}} \rho\right]\right) \rtimes S P\left(a_{1}, a_{2}, \ldots, a_{k-1}, a_{k+1}\right),
$$

let $\pi_{1}$ stand for an irreducible subrepresentation of

$$
\delta\left(\left[\nu^{-a_{k+1}+1} \rho, \nu^{a_{k+1}-1} \rho\right]\right) \rtimes S P\left(a_{1}, a_{2}, \ldots, a_{k}\right)
$$

which is not a subquotient of

$$
\delta\left(\left[\nu^{-a_{k}} \rho, \nu^{a_{k+1}-1} \rho\right]\right) \rtimes S P\left(a_{1}, a_{2}, \ldots, a_{k-1}, a_{k+1}-1\right) .
$$


(b) If $\pi$ is a subrepresentation of

$$
\delta\left(\left[\nu^{-a_{k}} \rho, \nu^{a_{k+1}} \rho\right]\right) \rtimes S P\left(a_{1}, a_{2}, \ldots, a_{k-1}, a_{k+1}\right),
$$

let $\pi_{1}$ stand for an irreducible subrepresentation of

$$
\delta\left(\left[\nu^{-a_{k+1}+1} \rho, \nu^{a_{k+1}-1} \rho\right]\right) \rtimes S P\left(a_{1}, a_{2}, \ldots, a_{k}\right)
$$

which is also a subquotient of

$$
\delta\left(\left[\nu^{-a_{k}} \rho, \nu^{a_{k+1}-1} \rho\right]\right) \rtimes S P\left(a_{1}, a_{2}, \ldots, a_{k-1}, a_{k+1}-1\right) .
$$

(ii) Suppose that $a_{k+1}=a_{k}+1$. Let $i_{\max }$ denote the maximal $i$ such that the Jacquet module of $\pi$ with respect to an appropriate parabolic subgroup contains an irreducible constituent of the form $\nu^{a_{i}} \rho \otimes \nu^{a_{i}+1} \rho \otimes \cdots \otimes$ $\nu^{a_{k+1}} \rho \otimes \nu^{a_{k+1}} \rho \otimes \sigma^{\prime}$.

(a) If $i_{\max }=k+1$, let $x_{l+1}=x_{l+2}=y_{l+1}=y_{l+2}=a_{k+1}$ and let $\pi_{1}^{\prime}$ denote an irreducible representation such that $\pi^{\prime} \hookrightarrow \nu^{-a_{k+1}} \rho \times$

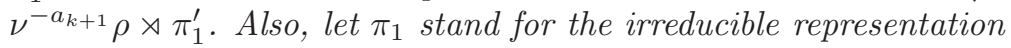

$$
\delta\left(\left[\nu^{-a_{k}} \rho, \nu^{a_{k}} \rho\right]\right) \rtimes S P\left(a_{1}, a_{2}, \ldots, a_{k}\right) .
$$

(b) If $i_{\max }<k+1$, let $x_{l+1}=y_{l+1}=a_{k+1}, x_{l+2}=a_{k+1}, y_{l+2}=a_{i_{\max }}$ and let $\pi_{1}^{\prime}$ denote an irreducible representation such that $\pi^{\prime} \hookrightarrow$ $\delta\left(\left[\nu^{-a_{k+1}} \rho, \nu^{-a_{i} \max } \rho\right]\right) \times \nu^{-a_{k+1}} \rho \rtimes \pi_{1}^{\prime}$. Also, let $\pi_{1}$ denote an irreducible subrepresentation of

$$
\delta\left(\left[\nu^{-a_{k}} \rho, \nu^{a_{k}} \rho\right]\right) \rtimes S P\left(a_{1}, a_{2}, \ldots, a_{i_{\max }-1}, a_{i_{\max }}-1, \ldots, a_{k}-1\right)
$$

which does not contain an irreducible constituent

$$
\begin{aligned}
& \nu^{a_{k}} \rho \times \nu^{a_{k}} \rho \otimes \delta\left(\left[\nu^{-a_{k}+1} \rho, \nu^{a_{k}-1} \rho\right]\right) \rtimes \\
& \quad S P\left(a_{1}, a_{2}, \ldots, a_{i_{\max }-1}, a_{i_{\max }}-1, \ldots, a_{k}-1\right)
\end{aligned}
$$

in the Jacquet module with respect to an appropriate parabolic subgroup.

Then $\left(T \cup\left\{\left(x_{l+1}, y_{l+1}\right),\left(x_{l+2}, y_{l+2}\right)\right\}, \pi_{1}, \pi_{1}^{\prime}\right)$ is a $(\sigma, \widehat{\sigma})$-triple.

Proof. To shorten the notation, let $\tau_{1}=\delta\left(\left[\nu^{-a_{k+1}} \rho, \nu^{a_{k+1}} \rho\right]\right) \rtimes S P\left(a_{1}\right.$, $\left.a_{2}, \ldots, a_{k}\right)$ throughout this proof. We note that the induced representation $\tau_{1}$ is a length two representation and both its irreducible subquotients are subrepresentations. Furthermore, there is exactly one irreducible subrepresentation of $\tau_{1}$ which contains

$\delta\left(\left[\nu^{a_{k}+1} \rho, \nu^{a_{k+1}} \rho\right]\right) \times \delta\left(\left[\nu^{a_{k}+1} \rho, \nu^{a_{k+1}} \rho\right]\right) \otimes \delta\left(\left[\nu^{-a_{k}} \rho, \nu^{a_{k}} \rho\right]\right) \rtimes S P\left(a_{1}, a_{2}, \ldots, a_{k}\right)$

in its Jacquet module with respect to an appropriate parabolic subgroup. Using [24, Theorem 4.1(iii)], one can conclude that such irreducible subrepresentation of $\tau_{1}$ is also a subrepresentation of

$$
\delta\left(\left[\nu^{-a_{k}} \rho, \nu^{a_{k+1}} \rho\right]\right) \rtimes S P\left(a_{1}, a_{2}, \ldots, a_{k-1}, a_{k+1}\right) .
$$


Let us first discuss the part $(i)$. By [24, Proposition 3.1], the induced representation $\nu^{-a_{k+1}} \rho \rtimes S P\left(a_{1}, a_{2}, \ldots, a_{k}\right)$ is irreducible. This provides the following embeddings and isomorphisms:

$$
\begin{aligned}
\pi & \hookrightarrow \tau_{1} \hookrightarrow \delta\left(\left[\nu^{-a_{k+1}-1} \rho, \nu^{a_{k+1}} \rho\right]\right) \times \nu^{-a_{k+1}} \rho \rtimes S P\left(a_{1}, a_{2}, \ldots, a_{k}\right) \\
& \cong \delta\left(\left[\nu^{-a_{k+1}-1} \rho, \nu^{a_{k+1}} \rho\right]\right) \times \nu^{a_{k+1}} \rho \rtimes S P\left(a_{1}, a_{2}, \ldots, a_{k}\right) \\
& \cong \nu^{a_{k+1}} \rho \times \delta\left(\left[\nu^{-a_{k+1}-1} \rho, \nu^{a_{k+1}} \rho\right]\right) \rtimes S P\left(a_{1}, a_{2}, \ldots, a_{k}\right) \\
& \hookrightarrow \nu^{a_{k+1}} \rho \times \nu^{a_{k+1}} \rho \times \delta\left(\left[\nu^{-a_{k+1}-1} \rho, \nu^{a_{k+1}-1} \rho\right]\right) \rtimes S P\left(a_{1}, a_{2}, \ldots, a_{k}\right) .
\end{aligned}
$$

By [22, Lemma 3.2], there is an irreducible representation $\pi_{1}$ such that $\pi \hookrightarrow$ $\nu^{a_{k+1}} \rho \times \nu^{a_{k+1}} \rho \rtimes \pi_{1}$, and $\mu^{*}(\pi) \geq \nu^{a_{k+1}} \rho \times \nu^{a_{k+1}} \rho \otimes \pi_{1}$. Applying the structural formula given in Lemma 2.2 , we obtain that $\pi_{1} \leq \delta\left(\left[\nu^{-a_{k+1}+1} \rho, \nu^{a_{k+1}-1} \rho\right]\right) \rtimes$ $S P\left(a_{1}, a_{2}, \ldots, a_{k}\right)$. Let us determine $\pi_{1}$ in terms of $\pi$.

Suppose that

$$
\begin{aligned}
& \mu^{*}(\pi) \geq \\
& \delta\left(\left[\nu^{a_{k}+1} \rho, \nu^{a_{k+1}} \rho\right]\right) \times \delta\left(\left[\nu^{a_{k}+1} \rho, \nu^{a_{k+1}} \rho\right]\right) \otimes \delta\left(\left[\nu^{-a_{k}} \rho, \nu^{a_{k}} \rho\right]\right) \rtimes S P\left(a_{1}, a_{2}, \ldots, a_{k}\right) .
\end{aligned}
$$

Since $\nu^{a_{k+1}} \rho$ does not appear in the cuspidal support of $\pi_{1}$, applying the structural formula to $\mu^{*}\left(\nu^{a_{k+1}} \rho \times \nu^{a_{k+1}} \rho \rtimes \pi_{1}\right)$, we conclude that there is an irreducible constituent $\delta \otimes \delta\left(\left[\nu^{-a_{k}} \rho, \nu^{a_{k}} \rho\right]\right) \rtimes S P\left(a_{1}, a_{2}, \ldots, a_{k}\right)$ of $\mu^{*}\left(\pi_{1}\right)$ such that

$$
\delta\left(\left[\nu^{a_{k}+1} \rho, \nu^{a_{k+1}} \rho\right]\right) \times \delta\left(\left[\nu^{a_{k}+1} \rho, \nu^{a_{k+1}} \rho\right]\right) \leq \nu^{a_{k+1}} \rho \times \nu^{a_{k+1}} \rho \times \delta .
$$

Since $\pi_{1} \leq \delta\left(\left[\nu^{-a_{k+1}+1} \rho, \nu^{a_{k+1}-1} \rho\right]\right) \rtimes S P\left(a_{1}, a_{2}, \ldots, a_{k}\right)$ and only such constituent of $\mu^{*}\left(\delta\left(\left[\nu^{-a_{k+1}+1} \rho, \nu^{a_{k+1}-1} \rho\right]\right) \rtimes S P\left(a_{1}, a_{2}, \ldots, a_{k}\right)\right)$ is

$$
\begin{aligned}
\delta\left(\left[\nu^{a_{k}+1} \rho, \nu^{a_{k+1}-1} \rho\right]\right) & \times \delta\left(\left[\nu^{a_{k}+1} \rho, \nu^{a_{k+1}-1} \rho\right]\right) \otimes \\
& \otimes \delta\left(\left[\nu^{-a_{k}} \rho, \nu^{a_{k}} \rho\right]\right) \rtimes S P\left(a_{1}, a_{2}, \ldots, a_{k}\right),
\end{aligned}
$$

it follows that $\pi_{1}$ is the unique irreducible subrepresentation of

$$
\delta\left(\left[\nu^{-a_{k+1}+1} \rho, \nu^{a_{k+1}-1} \rho\right]\right) \rtimes S P\left(a_{1}, a_{2}, \ldots, a_{k}\right)
$$

which contains the irreducible representation (4.3) in the Jacquet module with respect to an appropriate parabolic subgroup. In other words, $\pi_{1}$ is also a subquotient of $\delta\left(\left[\nu^{-a_{k}} \rho, \nu^{a_{k+1}-1} \rho\right]\right) \rtimes S P\left(a_{1}, a_{2}, \ldots, a_{k-1}, a_{k+1}-1\right)$.

Now suppose that $\mu^{*}(\pi)$ does not contain

$\delta\left(\left[\nu^{a_{k}+1} \rho, \nu^{a_{k+1}} \rho\right]\right) \times \delta\left(\left[\nu^{a_{k}+1} \rho, \nu^{a_{k+1}} \rho\right]\right) \otimes \delta\left(\left[\nu^{-a_{k}} \rho, \nu^{a_{k}} \rho\right]\right) \rtimes S P\left(a_{1}, a_{2}, \ldots, a_{k}\right)$.

Let us show that in this case $\pi_{1}$ is not a subquotient of $\delta\left(\left[\nu^{-a_{k}} \rho, \nu^{a_{k+1}-1} \rho\right]\right) \rtimes$ $S P\left(a_{1}, a_{2}, \ldots, a_{k-1}, a_{k+1}-1\right)$. Otherwise, since $\pi_{1}$ is an irreducible subquotient of $\delta\left(\left[\nu^{-a_{k+1}+1} \rho, \nu^{a_{k+1}-1} \rho\right]\right) \rtimes S P\left(a_{1}, a_{2}, \ldots, a_{k}\right), \mu^{*}\left(\pi_{1}\right)$ would contain the irreducible constituent (4.3) and, using transitivity of Jacquet modules, 
we conclude that the Jacquet module of $\pi$ with respect to an appropriate parabolic subgroup contains

$$
\begin{aligned}
& \nu^{a_{k+1}} \rho \otimes \nu^{a_{k+1}} \rho \otimes \nu^{a_{k+1}-1} \rho \otimes \nu^{a_{k+1}-1} \rho \otimes \cdots \otimes \nu^{a_{k}+1} \rho \otimes \nu^{a_{k}+1} \rho \otimes \\
& \otimes \delta\left(\left[\nu^{-a_{k}} \rho, \nu^{a_{k}} \rho\right]\right) \rtimes S P\left(a_{1}, a_{2}, \ldots, a_{k}\right) .
\end{aligned}
$$

But, this is impossible since the only irreducible representation of a general linear group containing $\nu^{a_{k+1}} \rho \otimes \nu^{a_{k+1}} \rho \otimes \nu^{a_{k+1}-1} \rho \otimes \nu^{a_{k+1}-1} \rho \otimes \cdots \otimes \nu^{a_{k}+1} \rho \otimes$ $\nu^{a_{k}+1} \rho$ in the Jacquet module with respect to an appropriate parabolic subgroup is $\delta\left(\left[\nu^{a_{k}+1} \rho, \nu^{a_{k+1}} \rho\right]\right) \times \delta\left(\left[\nu^{a_{k}+1} \rho, \nu^{a_{k+1}} \rho\right]\right)$.

Again, since $\nu^{a_{k+1}} \rho$ does not appear in the cuspidal support of $\pi_{1}$, we get that if $\nu^{a_{k+1}} \rho \otimes \nu^{a_{k+1}} \rho \otimes \pi_{2}$ appears in the Jacquet module of $\pi$ with respect to an appropriate parabolic subgroup, for some irreducible $\pi_{2}$, then $\pi_{2} \cong \pi_{1}$.

From Definition 4.1 we obtain that the Jacquet module of $\pi^{\prime}$ contains an irreducible constituent of the form $\nu^{-a_{k+1}} \rho \otimes \nu^{-a_{k+1}} \rho \otimes \pi^{\prime \prime}$, which implies that $\pi^{\prime}$ is a subrepresentation of $\nu^{-a_{k+1}} \rho \times \nu^{-a_{k+1}} \rho \rtimes \pi_{1}^{\prime}$, for some irreducible representation $\pi_{1}^{\prime}$. Now Lemma 4.4 and Lemma 4.6 imply that $\left(T \cup\left\{\left(x_{l+1}, y_{l+1}\right),\left(x_{l+2}, y_{l+2}\right)\right\}, \pi_{1}, \pi_{1}^{\prime}\right)$ is a $(\sigma, \widehat{\sigma})$-triple.

Let us now discuss the part $(i i)$.

If $i_{\max }=k+1$, the Jacquet module of $\pi$ with respect to an appropriate parabolic subgroup contains an irreducible constituent of the form $\nu^{a_{k+1}} \rho \otimes \nu^{a_{k+1}} \rho \otimes \sigma^{\prime}$. Since $\nu^{a_{k+1}} \rho \times \nu^{a_{k+1}} \rho$ is irreducible, using [22, Lemma 3.1, Lemma 3.2], we deduce that there is some irreducible representation $\pi_{1}$ such that $\pi$ is a subrepresentation of $\nu^{a_{k+1}} \rho \times \nu^{a_{k+1}} \rho \rtimes \pi_{1}$. Also, since $\nu^{a_{k+1}} \rho$ does not appear in the cuspidal support of $\pi_{1}$, if $\mu^{*}(\pi)$ contains an irreducible constituent of the form $\nu^{a_{k+1}} \rho \times \nu^{a_{k+1}} \rho \otimes \pi_{1}^{\prime}$, then $\pi_{1}^{\prime} \cong \pi_{1}$. Furthermore, using the structural formula given in Lemma 2.2 and irreducibility of $\delta\left(\left[\nu^{-a_{k}} \rho, \nu^{a_{k}} \rho\right]\right) \rtimes S P\left(a_{1}, a_{2}, \ldots, a_{k}\right)$, we obtain that the only irreducible constituent of the form $\nu^{a_{k+1}} \rho \times \nu^{a_{k+1}} \rho \otimes \sigma^{\prime}$ appearing in $\mu^{*}\left(\tau_{1}\right)$ is

$$
\nu^{a_{k+1}} \rho \times \nu^{a_{k+1}} \rho \otimes \delta\left(\left[\nu^{-a_{k}} \rho, \nu^{a_{k}} \rho\right]\right) \rtimes S P\left(a_{1}, a_{2}, \ldots, a_{k}\right) .
$$

Thus, $\pi_{1} \cong \delta\left(\left[\nu^{-a_{k}} \rho, \nu^{a_{k}} \rho\right]\right) \rtimes S P\left(a_{1}, a_{2}, \ldots, a_{k}\right)$. In the same way as in the proof of Lemma 4.6 we deduce that $\mu^{*}\left(\pi^{\prime}\right)$ contains an irreducible constituent of the form $\nu^{-a_{k+1}} \rho \times \nu^{-a_{k+1}} \rho \otimes \sigma^{\prime \prime}$. Using [22, Lemma 3.1, Lemma 3.2] and irreducibility of $\nu^{-a_{k+1}} \rho \times \nu^{-a_{k+1}} \rho$, one can see that there is an irreducible representation $\pi_{1}^{\prime}$ such that $\pi^{\prime}$ is a subrepresentation of $\nu^{-a_{k+1}} \rho \times \nu^{-a_{k+1}} \rho \rtimes \pi_{1}^{\prime}$. Since $\nu^{-a_{k+1}} \rho$ does not appear in the cuspidal support of $\pi_{1}^{\prime}$, and $\nu^{x} \rho$ appears in the cuspidal support of $\pi_{1}^{\prime}$ only for $-a_{k+1}+1 \leq x \leq a_{k+1}-1$, we see at once that $\left(T \cup\left\{\left(x_{l+1}, y_{l+1}\right),\left(x_{l+2}, y_{l+2}\right)\right\}, \pi_{1}, \pi_{1}^{\prime}\right)$ is a $(\sigma, \widehat{\sigma})$-triple.

Let us now assume that $i_{\max }<k+1$. In this case we have $a_{j+1}=a_{j}+1$ for $j=i_{\max }, \ldots, k$. Similarly as before, $\pi$ is a subrepresentation of an induced representation of the form $\nu^{a_{\text {max }}} \rho \times \nu^{a_{i_{\max }}+1} \rho \times \cdots \times \nu^{a_{k+1}} \rho \times \nu^{a_{k+1}} \rho \rtimes \pi_{1}$, for some irreducible representation $\pi_{1}$. Definition of $i_{\max }$ now implies that $\pi$ 
is also a subrepresentation of

$$
L\left(\nu^{a_{i_{\max }}} \rho, \nu^{a_{i_{\max }}+1} \rho, \ldots, \nu^{a_{k+1}} \rho\right) \times \nu^{a_{k+1}} \rho \rtimes \pi_{1} .
$$

Consequently, $\mu^{*}(\pi) \geq L\left(\nu^{a_{i_{\max }}} \rho, \nu^{a_{i_{\max }}+1} \rho, \ldots, \nu^{a_{k+1}} \rho\right) \times \nu^{a_{k+1}} \rho \otimes \pi_{1}$. Since $\pi$ is a subrepresentation of $\tau_{1}$, using the structural formula we deduce that there exist $-a_{k+1}-1 \leq i \leq j \leq a_{k+1}$ and an irreducible constituent $\delta \otimes \tau_{2}$ of $\mu^{*}\left(S P\left(a_{1}, a_{2}, \ldots, a_{k}\right)\right)$ such that

$$
L\left(\nu^{a_{i} \max } \rho, \ldots, \nu^{a_{k+1}} \rho\right) \times \nu^{a_{k+1}} \rho \leq \delta\left(\left[\nu^{-i} \rho, \nu^{a_{k+1}} \rho\right]\right) \times \delta\left(\left[\nu^{j} \rho, \nu^{a_{k+1}} \rho\right]\right) \times \delta
$$

and

$$
\pi_{1} \leq \delta\left(\left[\nu^{i+1} \rho, \nu^{j} \rho\right]\right) \rtimes \tau_{2} .
$$

From [14, Theorem 4.6] we conclude that $i \geq-a_{k+1}$ and $j \leq a_{k+1}$. Obviously, $i \leq-a_{i_{\max }}$ and $j \geq a_{i_{\max }}$. If either $i=-a_{i_{\max }}$ or $j=a_{i_{\max }}$, we have

$$
\delta\left(\left[\nu^{-i} \rho, \nu^{a_{k+1}} \rho\right]\right) \times \delta\left(\left[\nu^{j} \rho, \nu^{a_{k+1}} \rho\right]\right) \times \delta \cong \delta\left(\left[\nu^{a_{i \max }} \rho, \nu^{a_{k+1}} \rho\right]\right) \times \nu^{a_{k+1}} \rho,
$$

and this representation is irreducible and not isomorphic to the induced representation $L\left(\nu^{a_{i_{\max }}} \rho, \ldots, \nu^{a_{k+1}} \rho\right) \times \nu^{a_{k+1}} \rho$, since $i_{\max }<k+1$. Thus, $\nu^{a_{i_{\max }} \rho}$ appears in the cuspidal support of $\delta$ and [14, Theorem 4.6] implies that $\delta \cong L\left(\nu^{a_{i_{\max }}} \rho, \ldots, \nu^{a_{m}} \rho\right)$, for some $m \in\left\{i_{\max }, i_{\max }+1, \ldots, k\right\}$. Thus,

$$
\delta\left(\left[\nu^{-i} \rho, \nu^{a_{k+1}} \rho\right]\right) \times \delta\left(\left[\nu^{j} \rho, \nu^{a_{k+1}} \rho\right]\right) \times \delta \cong \delta\left(\left[\nu^{a_{m+1}} \rho, \nu^{a_{k+1}} \rho\right]\right) \times \nu^{a_{k+1}} \rho \times \delta .
$$

If $m \neq k$, using formula for $m^{*}$ we see that $\nu^{a_{i_{\max }} \rho \otimes \nu^{a_{i_{\max }+1}} \rho \otimes \cdots \otimes \nu^{a_{k}} \rho \otimes}$ $\nu^{a_{k+1}} \rho \otimes \nu^{a_{k+1}} \rho$ is not contained in the Jacquet module of $\delta\left(\left[\nu^{a_{m+1}} \rho, \nu^{a_{k+1}} \rho\right]\right) \times$ $\nu^{a_{k+1}} \rho \times \delta$. Consequently, $m=k, i=-a_{k+1}, j=a_{k+1}$ and $\pi_{1}$ is an irreducible subquotient of $\delta\left(\left[\nu^{-a_{k}} \rho, \nu^{a_{k}} \rho\right]\right) \rtimes S P\left(a_{1}, a_{2}, \ldots, a_{i_{\max }-1}, a_{i_{\max }}-1, \ldots, a_{k}-1\right)$.

Such induced representation has two irreducible subquotients, which are both subrepresentations and exactly one of them contains the irreducible constituent $\nu^{a_{k}} \rho \times \nu^{a_{k}} \rho \otimes \delta\left(\left[\nu^{-a_{k}+1} \rho, \nu^{a_{k}-1} \rho\right]\right) \rtimes S P\left(a_{1}, a_{2}, \ldots, a_{i_{\max }-1}, a_{i_{\max }}-\right.$ $\left.1, \ldots, a_{k}-1\right)$ in its Jacquet module with respect to an appropriate parabolic subgroup. Suppose that $\mu^{*}\left(\pi_{1}\right)$ contains

$\nu^{a_{k}} \rho \times \nu^{a_{k}} \rho \otimes \delta\left(\left[\nu^{-a_{k}+1} \rho, \nu^{a_{k}-1} \rho\right]\right) \rtimes S P\left(a_{1}, a_{2}, \ldots, a_{i_{\max }-1}, a_{i_{\max }}-1, \ldots, a_{k}-1\right)$.

Then $\pi_{1}$ is a subrepresentation of an induced representation of the form $\nu^{a_{k}} \rho \times$ $\nu^{a_{k}} \rho \rtimes \pi_{2}$. Thus, using Lemma 3.1 we obtain

$$
\begin{aligned}
& \pi \hookrightarrow L\left(\nu^{a_{i \max }} \rho, \nu^{a_{i_{\max }}+1} \rho, \ldots, \nu^{a_{k+1}} \rho\right) \times \nu^{a_{k+1}} \rho \rtimes \pi_{1} \\
& \hookrightarrow \nu^{a_{k+1}} \rho \times L\left(\nu^{a_{i_{\max }}} \rho, \nu^{a_{i_{\max }}+1} \rho, \ldots, \nu^{a_{k+1}} \rho\right) \times \nu^{a_{k}} \rho \times \nu^{a_{k}} \rho \rtimes \pi_{2} \\
& \hookrightarrow \nu^{a_{k+1}} \rho \times \nu^{a_{i_{\max }}} \rho \times \cdots \times \nu^{a_{k-1}} \rho \times L\left(\nu^{a_{k}} \rho, \nu^{a_{k+1}} \rho\right) \times \nu^{a_{k}} \rho \times \nu^{a_{k}} \rho \rtimes \pi_{2} \\
& \cong \nu^{a_{k+1}} \rho \times \nu^{a_{i_{\max }}} \rho \times \cdots \times \nu^{a_{k-1}} \rho \times \nu^{a_{k}} \rho \times \nu^{a_{k}} \rho \times L\left(\nu^{a_{k}} \rho, \nu^{a_{k+1}} \rho\right) \rtimes \pi_{2} \text {. }
\end{aligned}
$$


Since $L\left(\nu^{a_{k}} \rho, \nu^{a_{k+1}} \rho\right)$ is a subrepresentation of $\nu^{a_{k}} \rho \times \nu^{a_{k+1}} \rho$, Frobenius reciprocity shows that the Jacquet module of $\pi$ with respect to an appropriate parabolic subgroup contains

$$
\nu^{a_{k+1}} \rho \otimes \nu^{a_{i_{\max }}} \rho \otimes \cdots \otimes \nu^{a_{k-1}} \rho \otimes \nu^{a_{k}} \rho \times \nu^{a_{k}} \rho \times \nu^{a_{k}} \rho \otimes \nu^{a_{k+1}} \rho \otimes \pi_{2} .
$$

Transitivity of Jacquet modules and the structural formula for $\mu^{*}\left(\tau_{1}\right)$ imply that $\nu^{a_{k}} \rho \times \nu^{a_{k}} \rho \times \nu^{a_{k}} \rho \otimes \nu^{a_{k+1}} \rho \otimes \pi_{2}$ is contained in the Jacquet module of $\delta\left(\left[\nu^{-a_{k+1}} \rho, \nu^{a_{k}} \rho\right]\right) \rtimes S P\left(a_{1}, a_{2}, \ldots, a_{i_{\max }-1}, a_{i_{\max }}-1, \ldots, a_{k-1}-1, a_{k}\right)$, which is impossible. Thus, $\mu^{*}\left(\pi_{1}\right)$ does not contain the irreducible constituent

$\nu^{a_{k}} \rho \times \nu^{a_{k}} \rho \otimes \delta\left(\left[\nu^{-a_{k}+1} \rho, \nu^{a_{k}-1} \rho\right]\right) \rtimes S P\left(a_{1}, a_{2}, \ldots, a_{i_{\max }-1}, a_{i_{\max }}-1, \ldots, a_{k}-1\right)$.

In the similar way as before we conclude that $\pi^{\prime}$ is a subrepresentation of an induced representation of the form $\nu^{-a_{i_{\max }}} \rho \times \nu^{-a_{i_{\max }}-1} \rho \times \cdots \times \nu^{-a_{k+1}} \rho \times$

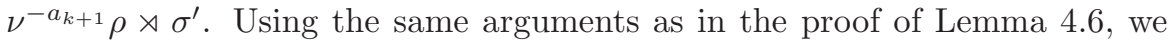
deduce that there is some irreducible representation $\pi_{1}^{\prime}$ such that

$$
\pi^{\prime} \hookrightarrow \delta\left(\left[\nu^{-a_{k+1}} \rho, \nu^{-a_{i \max }} \rho\right]\right) \times \nu^{-a_{k+1}} \rho \rtimes \pi_{1}^{\prime} .
$$

Using Lemma 4.4, one readily sees that $\left(T \cup\left\{\left(x_{l+1}, y_{l+1}\right),\left(x_{l+2}, y_{l+2}\right)\right\}, \pi_{1}\right.$, $\left.\pi_{1}^{\prime}\right)$ is a $(\sigma, \widehat{\sigma})$-triple.

LEMMA 4.10. Suppose that $\left(T, \pi, \pi^{\prime}\right)$ is a $(\sigma, \widehat{\sigma})$-triple and that $\pi$ is an irreducible subrepresentation of $\delta\left(\left[\nu^{-\alpha} \rho, \nu^{\alpha} \rho\right]\right) \rtimes \sigma_{\text {cusp }}$ such that $\mu^{*}(\pi)$ does not contain the irreducible constituent $\nu^{\alpha} \rho \times \nu^{\alpha} \rho \otimes \delta\left(\left[\nu^{-\alpha+1} \rho, \nu^{\alpha-1} \rho\right]\right) \rtimes \sigma_{\text {cusp }}$. Then $\pi^{\prime}$ is the unique irreducible subrepresentation of the induced representation

$$
\nu^{-\alpha} \rho \times \nu^{-\alpha+1} \rho \times \cdots \times \nu^{\lceil\alpha\rceil-\alpha-1} \rho \rtimes \tau_{\text {temp }}
$$

where $\tau_{\text {temp }}$ stands for

(i) $\operatorname{SP}\left(\frac{1}{2}, \frac{3}{2}, \ldots, \alpha\right)$, if $\alpha \neq\lceil\alpha\rceil$,

(ii) the unique irreducible tempered subrepresentation of $\rho \rtimes S P(1,2, \ldots, \alpha)$ which does not contain an irreducible representation of the form $\nu \rho \otimes \pi^{\prime \prime}$ in its Jacquet module with respect to an appropriate parabolic subgroup, if $\alpha=\lceil\alpha\rceil$.

Proof. Note that, by Lemma 2.2, if an irreducible constituent of the form $\nu^{x} \rho \otimes \sigma^{\prime}$ appears in $\mu^{*}(\pi)$, then $x=\alpha$. Also, no irreducible representation of the form $\nu^{\alpha} \rho \otimes \nu^{\alpha} \rho \otimes \sigma^{\prime}$ appears in the Jacquet module of $\pi$ with respect to an appropriate parabolic subgroup. Using Definition 4.1 and properties of the Aubert involution (Theorem $2.1(i v)$ ), we conclude that $\mu^{*}\left(\pi^{\prime}\right)$ does not contain an irreducible constituent of the form $\nu^{x} \rho \otimes \sigma_{1}^{\prime}$ for $x \neq-\alpha$, and that an irreducible constituent of the form $\nu^{-\alpha} \rho \otimes \nu^{-\alpha} \rho \otimes \sigma_{1}^{\prime}$ does not appear in the Jacquet module of $\pi^{\prime}$ with respect to an appropriate parabolic subgroup.

Obviously, $\mu^{*}(\pi) \geq \nu^{\alpha} \rho \otimes \sigma^{\prime}$ for some irreducible $\sigma^{\prime}$ and standard arguments show that there is some irreducible representation $\pi_{1}^{\prime}$ such that $\pi^{\prime} \hookrightarrow \nu^{-\alpha} \rho \rtimes \pi_{1}^{\prime}$. Let $\pi_{1}^{\prime}=L\left(\delta_{1}, \delta_{2}, \ldots, \delta_{m}, \tau\right)$ and $\delta_{i}=\delta\left(\left[\nu^{x_{i}} \rho, \nu^{y_{i}} \rho\right]\right)$. 
If $y_{1}>-\alpha+1$, we have

$$
\begin{aligned}
\pi^{\prime} & \hookrightarrow \nu^{-\alpha} \rho \times \delta_{1} \times \cdots \times \delta_{m} \rtimes \tau \\
& \hookrightarrow \nu^{-\alpha} \rho \times \nu^{y_{1}} \rho \times \delta\left(\left[\nu^{x_{1}} \rho, \nu^{y_{1}-1} \rho\right]\right) \times \delta_{2} \times \cdots \times \delta_{m} \rtimes \tau \\
& \cong \nu^{y_{1}} \rho \times \nu^{-\alpha} \rho \times \delta\left(\left[\nu^{x_{1}} \rho, \nu^{y_{1}-1} \rho\right]\right) \times \delta_{2} \times \cdots \times \delta_{m} \rtimes \tau,
\end{aligned}
$$

which gives $\mu^{*}\left(\pi^{\prime}\right) \geq \nu^{y_{1}} \rho \otimes \sigma^{\prime}$, for some irreducible $\sigma^{\prime}$, a contradiction. Similarly, if $y_{1}=-\alpha$ we have

$$
\pi^{\prime} \hookrightarrow \nu^{-\alpha} \rho \times \nu^{-\alpha} \rho \times \delta\left(\left[\nu^{x_{1}} \rho, \nu^{\alpha-1} \rho\right]\right) \times \delta_{2} \times \cdots \times \delta_{m} \rtimes \tau,
$$

which is also impossible. Thus, $y_{1}=-\alpha+1$. From the cuspidal support of $\pi^{\prime}$ we deduce that $x_{1} \in\{-\alpha,-\alpha+1\}$. If $x_{1}=-\alpha$, we have $\nu^{-\alpha} \rho \times \delta_{1} \hookrightarrow \nu^{-\alpha+1} \rho \times$ $\nu^{-\alpha} \rho \times \nu^{-\alpha} \rho$ which implies $\mu^{*}\left(\pi^{\prime}\right) \geq \nu^{-\alpha+1} \rho \otimes \sigma^{\prime}$, for some irreducible $\sigma^{\prime}$. Thus, $x_{1}=y_{1}=-\alpha+1$. We also have $\pi^{\prime} \hookrightarrow L\left(\nu^{-\alpha} \rho, \nu^{-\alpha+1} \rho\right) \times \delta_{2} \times \cdots \times$ $\delta_{m} \rtimes \tau$.

In the same way we conclude that $y_{2} \leq-\alpha+2$. From $e\left(\delta_{1}\right) \leq e\left(\delta_{2}\right)$, follows that $y_{2}>-\alpha$. If $y_{2}=-\alpha+1$, we have $\delta_{2} \hookrightarrow \nu^{-\alpha+1} \rho \times \delta^{\prime}$, for some irreducible $\delta^{\prime}$, and, using Lemma 3.1, we obtain that $\mu^{*}\left(\pi^{\prime}\right) \geq \nu^{-\alpha+1} \rho \otimes \sigma^{\prime}$, for some irreducible $\sigma^{\prime}$, a contradiction. Thus, $y_{2}=-\alpha+2$. If $x_{2} \leq-\alpha+1$, we have $\delta_{1} \times \delta_{2} \cong \delta_{2} \times \delta_{1}$ and in the same way as before we get $\mu^{*}\left(\pi^{\prime}\right) \geq$ $\nu^{-\alpha+2} \rho \otimes \sigma^{\prime}$, for some irreducible $\sigma^{\prime}$. Consequently, $x_{2}=y_{2}=-\alpha+2$ and $\pi$ is a subrepresentation of $L\left(\nu^{-\alpha} \rho, \nu^{-\alpha+1} \rho, \nu^{-\alpha+2} \rho\right) \times \delta_{3} \times \cdots \times \delta_{m} \rtimes \tau$. An inductive application of this procedure shows that $\delta_{i}=\nu^{-\alpha+i} \rho$ for $i=$ $1,2, \ldots, m$. Since $e\left(\delta_{i}\right)<0$ for all $i$, it follows that $-\alpha+m<0$.

If $\tau$ is not a strongly positive representation, there exist $b \geq a \geq 0$ such that $b-a$ is a non-negative integer and $\tau$ is a subrepresentation of $\delta\left(\left[\nu^{-a} \rho, \nu^{b} \rho\right]\right) \rtimes \tau^{\prime}$, for some irreducible representation $\tau^{\prime}$. If $b>0$, from the cuspidal support of $\pi^{\prime}$ we see that $\nu^{b-1} \rho$ does not appear in the cuspidal support of $\delta_{1} \times \delta_{2} \times \cdots \times \delta_{m}$. Thus, in this case we have $\tau \hookrightarrow \nu^{b} \rho \rtimes \sigma^{\prime}$ and $\delta_{i} \times \nu^{b} \rho \cong \nu^{b} \rho \times \delta_{i}$, leading to $\mu^{*}\left(\pi^{\prime}\right) \geq \nu^{b} \rho \otimes \sigma^{\prime \prime}$, for some irreducible $\sigma^{\prime \prime}$, a contradiction.

Consequently, if $\alpha \neq\lceil\alpha\rceil, \tau$ is a strongly positive representation. Since $\nu^{\frac{1}{2}} \rho$ can appear only once in the cuspidal support of a strongly positive representation, we get that $\delta_{m}=\nu^{-\frac{1}{2}} \rho$ and the cuspidal support of $\tau$ consists of $\nu^{\frac{1}{2}} \rho, \nu^{\frac{3}{2}} \rho, \ldots, \nu^{\alpha} \rho, \pi_{\text {cusp }}$, where $\pi_{\text {cusp }}$ denotes the partial cuspidal support of $\pi$. By [14, Lemma 3.5], a strongly positive representation is completely determined by its cuspidal support and, using the description of the strongly positive representations, we get $\tau \cong S P\left(\frac{1}{2}, \frac{3}{2}, \ldots, \alpha\right)$.

On the other hand, if $\alpha=\lceil\alpha\rceil$, since $\rho$ appears in neither $\delta_{1} \times \delta_{2} \times \cdots \times \delta_{m}$, nor in the strongly positive part, $\tau$ is a tempered representation which is a subrepresentation of an induced representation of the form $\rho \rtimes \sigma_{s p}$, for some strongly positive discrete series $\sigma_{s p}$. This also implies that, if $\alpha=\lceil\alpha\rceil$, then $\delta_{m}=\nu^{-1} \rho$, since otherwise we would have $\mu^{*}\left(\pi^{\prime}\right) \geq \rho \otimes \sigma^{\prime}$, for some 
irreducible $\sigma^{\prime}$. Thus, if $\alpha=\lceil\alpha\rceil$, the partial cuspidal support of $\sigma_{s p}$ consists of $\nu \rho, \nu^{2} \rho, \ldots, \nu^{\alpha} \rho, \pi_{\text {cusp }}$ and it follows that $\sigma_{s p} \cong S P(1,2, \ldots, \alpha)$.

The induced representation $\rho \rtimes \sigma_{s p}$ reduces, and it is a direct sum of two irreducible subrepresentations. By [28, Lemma 4.7, Corollary 4.9], there is a unique irreducible tempered subrepresentation of $\rho \rtimes S P(1,2, \ldots, \alpha)$ which contains an irreducible representation of the form $\nu \rho \otimes \pi^{\prime \prime}$ in its Jacquet module with respect to an appropriate parabolic subgroup. Such tempered representation is also a subrepresentation of an induced representation of the form $\nu \rho \rtimes \sigma^{\prime}$, for some irreducible $\sigma^{\prime}$. Since $\nu^{x} \rho \times \nu \rho \cong \nu \rho \times \nu^{x} \rho$ for $x<0$, the assumption that $\tau$ is a subrepresentation of $\nu \rho \rtimes \sigma^{\prime}$ leads to $\mu^{*}\left(\pi^{\prime}\right) \geq \nu \rho \otimes \sigma^{\prime \prime}$, for some irreducible $\sigma^{\prime \prime}$, which is impossible. Thus, $\tau$ is the unique irreducible tempered subrepresentation of $\rho \rtimes S P(1,2, \ldots, \alpha)$ which does not contain an irreducible representation of the form $\nu \rho \otimes \pi^{\prime \prime}$ in its Jacquet module with respect to an appropriate parabolic subgroup. This finishes the proof.

We are now ready to prove our first main result.

THEOREM 4.11. Suppose that a discrete series $\sigma$ is a subrepresentation of an induced representation of the form

$$
\delta\left(\left[\nu^{-a_{k+1}} \rho, \nu^{a_{k+2}} \rho\right]\right) \rtimes S P\left(a_{1}, a_{2}, \ldots, a_{k}\right),
$$

where $-1<a_{1}<a_{2}<\cdots<a_{k}<a_{k+1}<a_{k+2}$ are such that $\alpha-a_{i}$ is an integer for $i=1,2, \ldots, k+2$, and $\sigma$ is not a subrepresentation of the induced representation $\delta\left(\left[\nu^{-a_{k}} \rho, \nu^{a_{k+1}} \rho\right]\right) \rtimes S P\left(a_{1}, a_{2}, \ldots, a_{k-1}, a_{k+2}\right)$. Let $a_{0}=\alpha-$ $\lceil\alpha\rceil-1$.

Then the Aubert dual $\hat{\sigma}$ of $\sigma$ is the unique irreducible subrepresentation of the induced representation

$$
\prod_{i=-a_{k+2}}^{-a_{k+1}-1} \nu^{i} \rho \times\left(\prod_{i=1}^{k+1} \prod_{j=-a_{k-i+2}}^{-a_{k-i+1}-2} \delta\left(\left[\nu^{j-i+1} \rho, \nu^{j} \rho\right]\right) \times \nu^{j-i+1} \rho\right) \times \prod_{i=-\alpha}^{\lceil\alpha\rceil-\alpha-1} \nu^{i} \rho \rtimes \tau_{\text {temp }},
$$

where $\tau_{\text {temp }}$ stands for

(i) $\operatorname{SP}\left(\frac{1}{2}, \frac{3}{2}, \ldots, \alpha\right)$, if $\alpha \neq\lceil\alpha\rceil$,

(ii) the unique ireducible tempered subrepresentation of $\rho \rtimes S P(1,2, \ldots, \alpha)$ which does not contain an irreducible representation of the form $\nu \rho \otimes \pi^{\prime}$ in its Jacquet module with respect to an appropriate parabolic subgroup, if $\alpha=\lceil\alpha\rceil$.

Proof. Theorem follows from an inductive application of Lemmas 4.8, 4.9 and 4.10 , starting from the $(\sigma, \widehat{\sigma})$-triple $(\emptyset, \sigma, \widehat{\sigma})$. We note that it follows from the definition of the $(\sigma, \widehat{\sigma})$-triple that in each step $\widehat{\sigma}$ is obtained as an irreducible subrepresentation of the induced representation having a unique irreducible subrepresentation. 
First we are in the case of Lemma 4.8, and a repeated application of that lemma shows that $\widehat{\sigma}$ is a subrepresentation of $\prod_{i=-a_{k+2}}^{-a_{k+1}-1} \nu^{i} \rho \rtimes \pi_{1}^{\prime}$, for an irreducible representation $\pi_{1}^{\prime}$. Also, in the last application of Lemma 4.8, we end with a unique irreducible subrepresentation of $\delta\left(\left[\nu^{-a_{k+1}} \rho, \nu^{a_{k+1}} \rho\right]\right) \rtimes$ $S P\left(a_{1}, a_{2}, \ldots, a_{k}\right)$ which does not contain an irreducible constituent

$\delta\left(\left[\nu^{a_{k}+1} \rho, \nu^{a_{k+1}} \rho\right]\right) \times \delta\left(\left[\nu^{a_{k}+1} \rho, \nu^{a_{k+1}} \rho\right]\right) \otimes \delta\left(\left[\nu^{-a_{k}} \rho, \nu^{a_{k}} \rho\right]\right) \rtimes S P\left(a_{1}, a_{2}, \ldots, a_{k}\right)$ in its Jacquet module with respect to an appropriate parabolic subgroup.

This puts us in a situation described in Lemma 4.9. First we are in the part $(a)$ of the case $(i)$ of that lemma, which produces the part $\prod_{j=-a_{k+1}}^{-a_{k}-2} \nu^{j} \rho \times$ $\nu^{j} \rho$. We end with an irreducible subrepresentation of $\delta\left(\left[\nu^{-a_{k}+1} \rho, \nu^{a_{k}+1} \rho\right]\right) \rtimes$ $S P\left(a_{1}, a_{2}, \ldots, a_{k}\right)$ which is not a subquotient of the induced representation $\delta\left(\left[\nu^{-a_{k}} \rho, \nu^{a_{k}+1} \rho\right]\right) \rtimes S P\left(a_{1}, a_{2}, \ldots, a_{k-1}, a_{k}+1\right)$. This leads to the situation described in part $(b)$ of the case $(i i)$ of Lemma 4.9.

After $a_{k}-\alpha+1$ applications of that lemma, we obtain the part

$$
\prod_{i=2}^{k+1} \prod_{j=-a_{k-i+2}}^{-a_{k-i+1}-2} \delta\left(\left[\nu^{j-i+1} \rho, \nu^{j} \rho\right]\right) \times \nu^{j-i+1} \rho,
$$

written in a similar way as in [17, Section 3], using an approach analogous to the one used in Lemma 3.3.

Furthermore, after the last application of Lemma 4.9, we end with an irreducible subrepresentation of $\delta\left(\left[\nu^{-\alpha} \rho, \nu^{\alpha} \rho\right]\right) \rtimes \sigma_{\text {cusp }}$, which does not contain the irreducible constituent

$$
\nu^{\alpha} \rho \times \nu^{\alpha} \rho \otimes \delta\left(\left[\nu^{-\alpha+1} \rho, \nu^{\alpha-1} \rho\right]\right) \rtimes \sigma_{\text {cusp }}
$$

in the Jacquet module with respect to an appropriate parabolic subgroup. The rest of the proof now follows from Lemma 4.10.

We also note that if $a_{k+2}-a_{k+1} \in\{1,2\}$ then it follows from [5] that the unique irreducible subrepresentation of the induced representation (4.4) is unitarizable. If $\sigma$ is a subquotient of a principal series, considered groups are split, and $\operatorname{char} F=0$, it follows from [25] that the unique irreducible subrepresentation of the induced representation (4.4) is unitarizable.

Now we state and prove several results which enable us to handle the most complicated case.

Lemma 4.12. Suppose that $\left(T, \pi, \pi^{\prime}\right)$ is a $(\sigma, \widehat{\sigma})$-triple such that $\pi$ is a subrepresentation of both induced representations

$$
\delta\left(\left[\nu^{-a_{j+1}} \rho, \nu^{a_{j+2}} \rho\right]\right) \rtimes S P\left(a_{1}, \ldots, a_{j}, a_{j+3}, \ldots, a_{k+2}\right)
$$

and

$$
\delta\left(\left[\nu^{-a_{j}} \rho, \nu^{a_{j+1}} \rho\right]\right) \rtimes S P\left(a_{1}, \ldots, a_{j-1}, a_{j+2}, \ldots, a_{k+2}\right),
$$

where $-1<a_{1}<a_{2}<\cdots<a_{k+2}$ are such that $\alpha-a_{i}$ is an integer for $i=1,2, \ldots, k+2, j<k$ and $a_{j} \geq 0$. Let $l=|T|$. Let $i_{\max }$ denote the maximal 
$i$ such that the Jacquet module of $\pi$ with respect to an appropriate parabolic subgroup contains an irreducible constituent of the form $\nu^{a_{i}} \rho \otimes \nu^{a_{i+1}} \rho \otimes \cdots \otimes$ $\nu^{a_{k+2}} \rho \otimes \sigma^{\prime}$. Then $i_{\max } \geq j+2$. Let $x_{l+1}=a_{k+2}, y=a_{i_{\max }}$ and let $\pi_{1}^{\prime}$ denote an irreducible representation such that

$$
\pi^{\prime} \hookrightarrow \delta\left(\left[\nu^{-a_{k+2}} \rho, \nu^{-a_{i_{\max }}} \rho\right]\right) \rtimes \pi_{1}^{\prime} .
$$

If $i_{\max }>j+2$, let $\pi^{\prime \prime}$ denote

$\delta\left(\left[\nu^{-a_{j+1}} \rho, \nu^{a_{j+2}} \rho\right]\right) \rtimes S P\left(a_{1}, \ldots, a_{j}, a_{j+3}, \ldots, a_{i_{\max }-1}, a_{i_{\max }}-1, \ldots, a_{k+2}-1\right)$.

Otherwise, let $\pi^{\prime \prime}$ denote

$$
\delta\left(\left[\nu^{-a_{j+1}} \rho, \nu^{a_{j+2}-1} \rho\right]\right) \rtimes S P\left(a_{1}, \ldots, a_{j}, a_{j+3}-1, \ldots, a_{k+2}-1\right) .
$$

Let $\pi_{1}$ stand for the unique common irreducible subrepresentation of $\pi^{\prime \prime}$ and of

$\delta\left(\left[\nu^{-a_{j}} \rho, \nu^{a_{j+1}} \rho\right]\right) \rtimes S P\left(a_{1}, \ldots, a_{j-1}, a_{j+2}, \ldots, a_{i_{\max }-1}, a_{i_{\max }}-1, \ldots, a_{k+2}-1\right)$.

Then $\left(T \cup\left\{\left(x_{l+1}, y_{l+1}\right)\right\}, \pi_{1}, \pi_{1}^{\prime}\right)$ is a $(\sigma, \widehat{\sigma})$-triple.

Proof. Since $a_{j+3}>a_{j}+1$ by [12, Section 4] we have an embedding

$$
\begin{aligned}
S P\left(a_{1}, \ldots, a_{j}, a_{j+3}, \ldots, a_{k+2}\right) \hookrightarrow \nu^{a_{j+3}} \rho \times \nu^{a_{j+4}} \rho \times \cdots \times \nu^{a_{k+2}} \rho \rtimes \\
S P\left(a_{1}, \ldots, a_{j}, a_{j+3}-1, a_{k+2}-1\right),
\end{aligned}
$$

which provides another embedding and an isomorphism

$$
\begin{gathered}
\delta\left(\left[\nu^{-a_{j+1}} \rho, \nu^{a_{j+2}} \rho\right]\right) \rtimes S P\left(a_{1}, \ldots, a_{j}, a_{j+3}, \ldots, a_{k+2}\right) \hookrightarrow \\
\nu^{a_{j+2}} \rho \times \delta\left(\left[\nu^{-a_{j+1}} \rho, \nu^{a_{j+2}-1} \rho\right]\right) \times \nu^{a_{j+3}} \rho \times \nu^{a_{j+4}} \rho \times \cdots \times \nu^{a_{k+2}} \rho \rtimes \\
S P\left(a_{1}, \ldots, a_{j}, a_{j+3}-1, \ldots, a_{k+2}-1\right) \cong \\
\nu^{a_{j+2}} \rho \times \nu^{a_{j+3}} \rho \times \nu^{a_{j+4}} \rho \times \cdots \times \nu^{a_{k+2}} \rho \times \delta\left(\left[\nu^{-a_{j+1}} \rho, \nu^{a_{j+2}-1} \rho\right]\right) \rtimes \\
\quad S P\left(a_{1}, \ldots, a_{j}, a_{j+3}, \ldots, a_{k+2}-1\right) .
\end{gathered}
$$

Frobenius reciprocity gives $i_{\max } \geq j+2$. It remains to determine $\pi_{1}$, the rest of the proof then follows from Lemma 4.4 and Lemma 4.6.

There is some irreducible representation $\delta$ such that

$$
\begin{aligned}
& \mu^{*}\left(\delta\left(\left[\nu^{-a_{j+1}} \rho, \nu^{a_{j+2}} \rho\right]\right) \rtimes S P\left(a_{1}, \ldots, a_{j}, a_{j+3}, \ldots, a_{k+2}\right)\right) \geq \delta \otimes \pi_{1}, \\
& \mu^{*}\left(\delta\left(\left[\nu^{-a_{j}} \rho, \nu^{a_{j+1}} \rho\right]\right) \rtimes S P\left(a_{1}, \ldots, a_{j-1}, a_{j+2}, \ldots, a_{k+2}\right)\right) \geq \delta \otimes \pi_{1},
\end{aligned}
$$

and the Jacquet module of $\delta$ with respect to an appropriate parabolic subgroup contains $\nu^{a_{\max }} \rho \otimes \nu^{a_{i_{\max }+1}} \rho \otimes \cdots \otimes \nu^{a_{k+2}} \rho$. Using the structural formula and [14, Theorem 4.6], we obtain that either $\delta$ is isomorphic to the representation $L\left(\nu^{a_{i_{\max }}} \rho, \nu^{a_{i_{\max }+1}} \rho, \ldots, \nu^{a_{k+2}} \rho\right)$ (if $\left.i_{\max }>j+2\right)$, or $\delta$ is an irreducible subquotient of $\nu^{a_{j+2}} \rho \times L\left(\nu^{a_{j+3}} \rho, \nu^{a_{j+4}} \rho, \ldots, \nu^{a_{k+2}} \rho\right)$ (if $\left.i_{\max }=j+2\right)$.

In the first case, $\pi_{1}$ is a common irreducible subquotient of

$\delta\left(\left[\nu^{-a_{j+1}} \rho, \nu^{a_{j+2}} \rho\right]\right) \rtimes S P\left(a_{1}, \ldots, a_{j}, a_{j+3}, \ldots, a_{i_{\max }-1}, a_{i_{\max }}-1, \ldots, a_{k+2}-1\right)$ 
and of

$\delta\left(\left[\nu^{-a_{j}} \rho, \nu^{a_{j+1}} \rho\right]\right) \rtimes S P\left(a_{1}, \ldots, a_{j-1}, a_{j+2}, \ldots, a_{i_{\max }-1}, a_{i_{\max }}-1, \ldots, a_{k+2}-1\right)$.

Otherwise, $\pi_{1}$ is a common irreducible subquotient of

$$
\delta\left(\left[\nu^{-a_{j+1}} \rho, \nu^{a_{j+2}-1} \rho\right]\right) \rtimes S P\left(a_{1}, \ldots, a_{j}, a_{j+3}-1, \ldots, a_{k+2}-1\right)
$$

and of

$$
\delta\left(\left[\nu^{-a_{j}} \rho, \nu^{a_{j+1}} \rho\right]\right) \rtimes S P\left(a_{1}, \ldots, a_{j-1}, a_{j+2}-1, \ldots, a_{k+2}-1\right) .
$$

Now Theorem 2.3 and [24, Theorem 4.1(iii)] imply that the representation $\pi_{1}$ is completely determined in this way and, if there is some irreducible representation $\pi_{2}$ such that the Jacquet module of $\pi$ with respect to an appropriate parabolic subgroup contains $\nu^{a_{i_{\max }}} \rho \otimes \nu^{a_{i_{\max }+1}} \rho \otimes \cdots \otimes \nu^{a_{k+2}} \rho \otimes \pi_{2}$, then $\pi_{2} \cong \pi_{1}$.

Lemma 4.13. Suppose that $\left(T, \pi, \pi^{\prime}\right)$ is a $(\sigma, \widehat{\sigma})$-triple such that $\pi$ is a unique common irreducible subquotient of

$$
\delta\left(\left[\nu^{-a} \rho, \nu^{a} \rho\right]\right) \rtimes S P\left(a_{1}, a_{2}, \ldots, a_{i}, a+1, \ldots, a+k-i\right)
$$

and of

$$
\delta\left(\left[\nu^{-a_{i}} \rho, \nu^{a} \rho\right]\right) \rtimes S P\left(a_{1}, a_{2}, \ldots, a_{i-1}, a, a+1, \ldots, a+k-i\right),
$$

for $-1<a_{1}<a_{2}<\cdots<a_{i}<a$ such that $\alpha-a_{j}$ is an integer for $j=$ $1,2, \ldots, i$, and such that $a-a_{i}$ is an integer. Let $l=|T|$. Let $x_{l+1}=a+k-i$, $y_{l+1}=a, x_{l+2}=y_{l+2}=a$ and let $\pi_{1}^{\prime}$ denote an irreducible representation such that $\pi^{\prime} \hookrightarrow \delta\left(\left[\nu^{-a-k+i} \rho, \nu^{-a} \rho\right]\right) \times \nu^{-a} \rho \rtimes \pi_{1}^{\prime}$. Also, let $\pi_{1}$ denote the unique common irreducible subquotient of

$$
\delta\left(\left[\nu^{-a+1} \rho, \nu^{a-1} \rho\right]\right) \rtimes S P\left(a_{1}, a_{2}, \ldots, a_{i}, a, a+1 \ldots, a+k-i-1\right)
$$

and

$$
\delta\left(\left[\nu^{-a_{i}} \rho, \nu^{a-1} \rho\right]\right) \rtimes S P\left(a_{1}, a_{2}, \ldots, a_{i-1}, a-1, a, \ldots, a+k-i-1\right) .
$$

Then $\left(T \cup\left\{\left(x_{l+1}, y_{l+1}\right),\left(x_{l+2}, y_{l+2}\right)\right\}, \pi_{1}, \pi_{1}^{\prime}\right)$ is a $(\sigma, \widehat{\sigma})$-triple. We note that if $a_{i}=a-1$, then the previous two induced representations are mutually isomorphic and irreducible.

Proof. Clearly, $\pi$ is a tempered subrepresentation of the induced representation $\delta\left(\left[\nu^{-a} \rho, \nu^{a} \rho\right]\right) \rtimes S P\left(a_{1}, a_{2}, \ldots, a_{i}, a+1, \ldots, a+k-i\right)$, and we have the following embeddings and isomorphism:

$$
\begin{aligned}
\pi \hookrightarrow & \delta\left(\left[\nu^{-a} \rho, \nu^{a} \rho\right]\right) \rtimes S P\left(a_{1}, a_{2}, \ldots, a_{i}, a+1, \ldots, a+k-i\right) \\
\hookrightarrow & \nu^{a} \rho \times \delta\left(\left[\nu^{-a} \rho, \nu^{a-1} \rho\right]\right) \times \nu^{a+1} \rho \times \cdots \times \nu^{a+k-i} \rho \rtimes \\
& S P\left(a_{1}, a_{2}, \ldots, a_{i}, a, \ldots, a+k-i-1\right) \\
\simeq & \nu^{a} \rho \times \nu^{a+1} \rho \times \cdots \times \nu^{a+k-i} \rho \times \delta\left(\left[\nu^{-a} \rho, \nu^{a-1} \rho\right]\right) \rtimes \\
& S P\left(a_{1}, a_{2}, \ldots, a_{i}, a, \ldots, a+k-i-1\right) .
\end{aligned}
$$


From [24, Theorem 4.1(iii)] one can see that $\pi$ is also a subrepresentation of the induced representation $\delta\left(\left[\nu^{-a_{i}} \rho, \nu^{a} \rho\right]\right) \rtimes S P\left(a_{1}, a_{2}, \ldots, a_{i-1}, a, a+1, \ldots, a+\right.$ $k-i)$, so we have:

$$
\begin{aligned}
\pi \hookrightarrow & \delta\left(\left[\nu^{-a_{i}} \rho, \nu^{a} \rho\right]\right) \rtimes S P\left(a_{1}, a_{2}, \ldots, a_{i-1}, a, a+1, \ldots, a+k-i\right) \\
\hookrightarrow & \delta\left(\left[\nu^{-a_{i}} \rho, \nu^{a} \rho\right]\right) \times \nu^{a} \rho \rtimes S P\left(a_{1}, a_{2}, \ldots, a_{i-1}, a-1, a+1, \ldots, a+k-i\right) \\
\hookrightarrow & \nu^{a} \rho \times \nu^{a} \rho \times \delta\left(\left[\nu^{-a_{i}} \rho, \nu^{a-1} \rho\right]\right) \rtimes \\
& S P\left(a_{1}, a_{2}, \ldots, a_{i-1}, a-1, a+1, \ldots, a+k-i\right) \\
\hookrightarrow & \nu^{a} \rho \times \nu^{a} \rho \times \nu^{a+1} \rho \times \cdots \times \nu^{a+k-i} \rho \times \delta\left(\left[\nu^{-a} \rho, \nu^{a-1} \rho\right]\right) \rtimes \\
& S P\left(a_{1}, a_{2}, \ldots, a_{i}, a-1, \ldots, a+k-i-1\right) .
\end{aligned}
$$

It follows that $\mu^{*}\left(\delta\left(\left[\nu^{-a} \rho, \nu^{a-1} \rho\right]\right) \rtimes S P\left(a_{1}, a_{2}, \ldots, a_{i}, a, \ldots, a+k-i-1\right)\right)$ contains an irreducible constituent of the form $\nu^{a} \rho \otimes \sigma_{1}$, and $\pi$ is a subrepresentation of an induced representation of the form $\nu^{a} \rho \times \nu^{a+1} \rho \times \cdots \times \nu^{a+k-i} \rho \times$ $\nu^{a} \rho \rtimes \sigma_{2}$. Thus, Frobenius reciprocity shows that the Jacquet module of $\pi$ with respect to an appropriate parabolic subgroup contains an irreducible representation of the form $\nu^{a} \rho \otimes \nu^{a+1} \rho \otimes \cdots \otimes \nu^{a+k-i} \rho \otimes \nu^{a} \rho \otimes \pi_{1}$. From the embedding $\pi \hookrightarrow \delta\left(\left[\nu^{-a_{i}} \rho, \nu^{a} \rho\right]\right) \rtimes S P\left(a_{1}, a_{2}, \ldots, a_{i-1}, a, a+1, \ldots, a+k-i\right)$ we also obtain that $a$ is the maximal $j$ such that the Jacquet module of $\pi$ with respect to an appropriate parabolic subgroup contains an irreducible representation of the form $\nu^{j} \rho \otimes \nu^{j+1} \rho \otimes \cdots \otimes \nu^{a+k-i} \rho \otimes \sigma_{3}$. Furthermore, using the same embedding we deduce that $\mu^{*}(\pi)$ does not contain an irreducible constituent of the form $\nu^{a} \rho \times \nu^{a} \rho \times \nu^{a} \rho \otimes \sigma_{4}$. Obviously, $\nu^{a+k-i} \rho$ does not appear in the cuspidal support of $\pi_{1}$.

In the same way as in the proof of the previous lemma one can determine $\pi_{1}$, and in the same way as in the proof of Lemma 3.4 we deduce that $\pi^{\prime}$ is a subrepresentation of an induced representation of the form $\delta\left(\left[\nu^{-a-k+i} \rho, \nu^{-a} \rho\right]\right) \times \nu^{-a} \rho \rtimes \pi_{1}^{\prime}$, for some irreducible representation $\pi_{1}^{\prime}$. Now in the same way as in the proof of the previous lemma one can show that $\left(T \cup\left\{\left(x_{l+1}, y_{l+1}\right),\left(x_{l+2}, y_{l+2}\right)\right\}, \pi_{1}, \pi_{1}^{\prime}\right)$ satisfies the property $(a)$ of Definition 4.1 .

Also, $\mu^{*}\left(\pi_{1}^{\prime}\right)$ does not contain an irreducible constituent of the form $\nu^{-a} \rho \otimes \sigma_{4}$, since otherwise we would have an embedding of the form

$$
\pi^{\prime} \hookrightarrow \nu^{-a} \rho \times \nu^{-a} \rho \times \nu^{-a} \rho \rtimes \sigma_{5}
$$

which would lead to an embedding

$$
\pi \hookrightarrow \nu^{a} \rho \times \nu^{a} \rho \times \nu^{a} \rho \rtimes \sigma_{6},
$$

a contradiction. From Lemma 4.5 we deduce that $\left(T \cup\left\{\left(x_{l+1}, y_{l+1}\right)\right.\right.$, $\left.\left.\left(x_{l+2}, y_{l+2}\right)\right\}, \pi_{1}, \pi_{1}^{\prime}\right)$ satisfies the property (b3) of Definition 4.1 , and it obviously satisfies the property $(b 1)$. It remains to check the property $(b 2)$, so let $\pi_{1}^{\prime}=L\left(\delta_{1}^{\prime}, \delta_{2}^{\prime}, \ldots, \delta_{r}^{\prime}, \tau\right)$. Using Lemma 4.6 we conclude that we only need to check that if $e\left(\delta_{j}^{\prime}\right)<-a$, for some $j \in\{1,2, \ldots, r\}$, then $\nu^{-a} \rho \times \delta_{j}^{\prime} \cong \delta_{j}^{\prime} \times \nu^{-a} \rho$. 
Write $\delta_{j}^{\prime}=\delta\left(\left[\nu^{x_{j}} \rho, \nu^{y_{j}} \rho\right]\right)$. If $e\left(\delta_{j}^{\prime}\right)<-a$, it follows at once that $x_{j}<-a$. Suppose that there is some $j \in\{1,2, \ldots, r\}$ such that $e\left(\delta_{j}^{\prime}\right)<-a$ and $\nu^{-a} \rho \times \delta_{j}^{\prime}$ is not isomorphic to $\delta_{j}^{\prime} \times \nu^{-a} \rho$. Let us denote the minimal such $j$ by $j_{\min }$. Then we have $y_{j_{\min }}=-a-1$. For $j=1,2, \ldots, j_{\min }-1$, since $e\left(\delta_{j}^{\prime}\right) \leq e\left(\delta_{j+1}^{\prime}\right)$ and $\delta_{j}^{\prime} \hookrightarrow \nu^{y_{j}} \rho \times \delta\left(\left[\nu^{x_{j}} \rho, \nu^{y_{j}-1} \rho\right]\right)$, we have either $y_{j} \leq y_{j+1}$ or $\delta_{j}^{\prime} \times \nu^{y_{j+1}} \rho \cong$ $\nu^{y_{j+1}} \rho \times \delta_{j}^{\prime}$. This enables us to conclude that $\pi_{1}^{\prime}$ is a subrepresentation of $\nu^{y} \rho \rtimes \pi_{1}^{\prime \prime}$, for $y \leq-a-1$ and an irreducible representation $\pi_{1}^{\prime \prime}$. Consequently, the Jacquet module of $\pi^{\prime}$ with respect to an appropriate parabolic subgroup contains $\nu^{-a} \rho \otimes \cdots \otimes \nu^{-a-k+i} \rho \otimes \nu^{-a} \rho \otimes \nu^{y} \rho \otimes \pi_{1}^{\prime \prime}$.

Using the property $(a)$ of Definition 4.1 , which has already been checked, we deduce that $\mu^{*}\left(\pi_{1}\right) \geq \nu^{-y} \rho \otimes \pi_{2}$, for some irreducible representation $\pi_{2}$. But, since $\pi_{1}$ is a subquotient of $\delta\left(\left[\nu^{-a+1} \rho, \nu^{a-1} \rho\right]\right) \rtimes S P\left(a_{1}, a_{2}, \ldots, a_{i}, a, a+\right.$ $1 \ldots, a+k-i-1)$, from the structural formula and [14, Theorem 4.6] follows that $\mu^{*}\left(\pi_{1}\right)$ does not contain irreducible constituent of the form $\nu^{x} \rho \otimes \pi_{2}$ for $x \geq a+1$, so it can not contain $\nu^{-y} \rho \otimes \pi_{2}$.

Consequently, $\left(T \cup\left\{\left(x_{l+1}, y_{l+1}\right),\left(x_{l+2}, y_{l+2}\right)\right\}, \pi_{1}, \pi_{1}^{\prime}\right)$ satisfies the property $(b 2)$ of Definition 4.1 and $\left(T \cup\left\{\left(x_{l+1}, y_{l+1}\right),\left(x_{l+2}, y_{l+2}\right)\right\}, \pi_{1}, \pi_{1}^{\prime}\right)$ is a $(\sigma, \widehat{\sigma})$-triple.

Lemma 4.14. Suppose that $\left(T, \pi, \pi^{\prime}\right)$ is a $(\sigma, \widehat{\sigma})$-triple such that

$$
\pi \cong \delta\left(\left[\nu^{-a} \rho, \nu^{a} \rho\right]\right) \rtimes S P\left(a_{1}, a_{2}, \ldots, a_{k}\right),
$$

where $-1<a_{1}<a_{2}<\cdots<a_{k}, \alpha-a_{i}$ is an integer for $i=1,2, \ldots, k$, $a_{k} \geq \alpha$, and there is some $j \in\{1,2, \ldots, k\}$ such that $a_{j}=a$ and $a_{i+1}=a_{i}+1$ for $i \geq j$. Let $l=|T|$. Let $i_{\max }$ denote the maximal $i$ such that the Jacquet module of $\pi$ with respect to an appropriate parabolic subgroup contains an irreducible constituent of the form $\nu^{a_{i}} \rho \otimes \nu^{a_{i+1}} \rho \otimes \cdots \otimes \nu^{a_{k}} \rho \otimes \nu^{a} \rho \otimes \nu^{a} \rho \otimes \sigma^{\prime}$. Let $x_{l+1}=a_{k}, y_{l+1}=a_{i_{\max }}, x_{l+2}=y_{l+2}=a, x_{l+3}=y_{l+3}=a$ and let $\pi_{1}^{\prime}$ denote an irreducible representation such that $\pi^{\prime} \hookrightarrow \delta\left(\left[\nu^{-a_{k}} \rho, \nu^{\left.\left.-a_{i_{\max }} \rho\right]\right) \times}\right.\right.$ $\nu^{-a} \rho \times \nu^{-a} \rho \rtimes \pi_{1}^{\prime}$. Also, let $\pi_{1}$ denote the irreducible representation

$$
\delta\left(\left[\nu^{-a+1} \rho, \nu^{a-1} \rho\right]\right) \rtimes S P\left(a_{1}, a_{2}, \ldots, a_{i_{\max }-1}, a_{i_{\max }}-1, \ldots, a_{k}-1\right)
$$

Then $\left(T \cup\left\{\left(x_{l+1}, y_{l+1}\right),\left(x_{l+2}, y_{l+2}\right),\left(x_{l+3}, y_{l+3}\right)\right\}, \pi_{1}, \pi_{1}^{\prime}\right)$ is a $(\sigma, \widehat{\sigma})$-triple.

Proof. It can be deduced using [22, Proposition 2.1] that the induced representation $\delta\left(\left[\nu^{-a} \rho, \nu^{a} \rho\right]\right) \rtimes S P\left(a_{1}, a_{2}, \ldots, a_{k}\right)$ is irreducible. Thus, there is an $i$ such that $\mu^{*}(\pi)$ contains an irreducible constituent

$$
\begin{aligned}
& L\left(a_{i}, a_{i+1}, \ldots, a_{k}\right) \times \nu^{a} \rho \times \nu^{a} \rho \otimes \\
& \delta\left(\left[\nu^{-a+1} \rho, \nu^{a-1} \rho\right]\right) \rtimes S P\left(a_{1}, a_{2}, \ldots, a_{i-1}, a_{i}-1, \ldots, a_{k}-1\right),
\end{aligned}
$$

where $a_{i} \leq a$. The rest of the proof now follows exactly as in the proof of Lemma 4.13 . 
Lemma 4.15. Suppose that $\left(T, \pi, \pi^{\prime}\right)$ is a $(\sigma, \widehat{\sigma})$-triple such that

$$
\pi \cong \delta\left(\left[\nu^{-a} \rho, \nu^{a} \rho\right]\right) \rtimes \sigma_{\text {cusp }},
$$

where $a<\alpha$ and $\alpha-a \in \mathbb{Z}$. Then $\pi^{\prime}$ is the unique irreducible subrepresentation of the induced representation

$$
\nu^{-a} \rho \times \nu^{-a} \rho \times \nu^{-a+1} \rho \times \nu^{-a+1} \rho \times \cdots \times \nu^{\lceil\alpha\rceil-\alpha-1} \rho \times \nu^{\lceil\alpha\rceil-\alpha-1} \rho \rtimes \tau_{\text {temp }},
$$

where $\tau_{\text {temp }}$ stands for

(i) $\sigma_{\text {cusp }}$, if $\alpha \neq\lceil\alpha\rceil$,

(ii) $\rho \rtimes \sigma_{\text {cusp }}$, if $\alpha=\lceil\alpha\rceil$.

Proof. For $0<x<\alpha$ such that $\alpha-x \in \mathbb{Z}$, we have the following embeddings and isomorphisms:

$$
\begin{aligned}
\delta\left(\left[\nu^{-x} \rho, \nu^{x} \rho\right]\right) \rtimes \sigma_{\text {cusp }} & \hookrightarrow \delta\left(\left[\nu^{-x+1} \rho, \nu^{x} \rho\right]\right) \times \nu^{-x} \rho \rtimes \sigma_{\text {cusp }} \\
& \cong \delta\left(\left[\nu^{-x+1} \rho, \nu^{x} \rho\right]\right) \times \nu^{x} \rho \rtimes \sigma_{\text {cusp }} \\
& \cong \nu^{x} \rho \times \delta\left(\left[\nu^{-x+1} \rho, \nu^{x} \rho\right]\right) \rtimes \sigma_{\text {cusp }} \\
& \hookrightarrow \nu^{x} \rho \times \nu^{x} \rho \times \delta\left(\left[\nu^{-x+1} \rho, \nu^{x-1} \rho\right]\right) \rtimes \sigma_{\text {cusp }} .
\end{aligned}
$$

Two possibilities will be studied separately. First, let $\alpha \neq\lceil\alpha\rceil$. A repeated application of the previous procedure gives an embedding

$$
\pi \hookrightarrow \nu^{a} \rho \times \nu^{a} \rho \times \nu^{a-1} \rho \times \nu^{a-1} \rho \times \cdots \times \nu^{\frac{1}{2}} \rho \times \nu^{\frac{1}{2}} \rho \rtimes \sigma_{\text {cusp }} .
$$

In the same way as before, we deduce that the Jacquet module of $\pi^{\prime}$ with respect to an appropriate parabolic subgroup contains $\nu^{-a} \rho \otimes \nu^{-a} \rho \otimes \nu^{-a+1} \rho \otimes$ $\nu^{-a+1} \rho \otimes \cdots \otimes \nu^{-\frac{1}{2}} \rho \otimes \nu^{-\frac{1}{2}} \rho \otimes \sigma_{\text {cusp }}$, and, using [22, Lemma 3.1], we see that $\pi^{\prime}$ is a subrepresentation of

$$
\nu^{-a} \rho \times \nu^{-a} \rho \times \nu^{-a+1} \rho \times \nu^{-a+1} \rho \times \cdots \times \nu^{-\frac{1}{2}} \rho \times \nu^{-\frac{1}{2}} \rho \rtimes \sigma_{\text {cusp }} .
$$

Since the induced representation (4.5) has a unique irreducible (Langlands) subrepresentation, this completely determines $\pi^{\prime}$ in this case.

Second, let $\alpha=\lceil\alpha\rceil$. In the same way as in the first case we conclude that $\pi^{\prime}$ is a subrepresentation of

(4.6) $\quad \nu^{-a} \rho \times \nu^{-a} \rho \times \nu^{-a+1} \rho \times \nu^{-a+1} \rho \times \cdots \times \nu^{-1} \rho \times \nu^{-1} \rho \rtimes\left(\rho \rtimes \sigma_{\text {cusp }}\right)$.

We note that the induced representation $\rho \rtimes \sigma_{\text {cusp }}$ is irreducible and tempered, so $\pi^{\prime}$ is the unique irreducible (Langlands) subrepresentation of (4.6), and the lemma is proved.

The previous sequence of lemmas leads to our second main result.

THEOREM 4.16. Suppose that a discrete series $\sigma$ is a subrepresentation of both induced representations

$$
\delta\left(\left[\nu^{-a_{m-1}} \rho, \nu^{a_{m}} \rho\right]\right) \rtimes S P\left(a_{1}, a_{2}, \ldots, a_{m-2}, a_{m+1}, \ldots, a_{k+2}\right),
$$


and

$$
\delta\left(\left[\nu^{-a_{m-2}} \rho, \nu^{a_{m-1}} \rho\right]\right) \rtimes S P\left(a_{1}, a_{2}, \ldots, a_{m-3}, a_{m}, \ldots, a_{k+2}\right),
$$

such that $\alpha-a_{i}$ is an integer for $i=1,2, \ldots, k+2,-1<a_{1}<a_{2}<\cdots<$ $a_{k}<a_{k+1}<a_{k+2}, m \geq 3$ and $a_{1} \geq 0$ if $m=3$. Let $a_{0}=\alpha-\lceil\alpha\rceil-1$. If there exists an $i, k-m+3 \leq i \leq k$, such that $a_{k-i+1} \geq a_{k-i}+2$, let $r=k-m-\alpha+3$, and otherwise let $r=-a_{m-2}$. Also, let $\tau_{\text {temp }}$ denote $\sigma_{\text {cusp }}$ if $\alpha \neq\lceil\alpha\rceil$ and let $\tau_{\text {temp }}$ denote $\rho \rtimes \sigma_{\text {cusp }}$ if $\alpha=\lceil\alpha\rceil$. The Aubert dual $\widehat{\sigma}$ of $\sigma$ is the unique irreducible subrepresentation of the induced representation

$$
\begin{gathered}
\prod_{i=1}^{k-m+2} \prod_{j=-a_{k-i+3}}^{-a_{k-i+2}-2} \delta\left(\left[\nu^{j-i+1} \rho, \nu^{j} \rho\right]\right) \times \prod_{j=-a_{m}}^{-a_{m-1}-1} \delta\left(\left[\nu^{j-k+m-2} \rho, \nu^{j} \rho\right]\right) \times \\
\times \prod_{j=-a_{m-1}}^{-a_{m-2}-1}\left(\delta\left(\left[\nu^{j-k+m-2} \rho, \nu^{j} \rho\right]\right) \times \nu^{j} \rho\right) \times \\
\prod_{i=k-m+3}^{k} \prod_{j=-a_{k-i+1}}^{-a_{k-i}-2}\left(\delta\left(\left[\nu^{j-i+1} \rho, \nu^{j} \rho\right]\right) \times \nu^{j-i+k-m+3} \rho \times \nu^{j-i+k-m+3} \rho\right) \times \\
\times \prod_{i=r}^{\lceil\alpha\rceil-\alpha-1}\left(\nu^{i} \rho \times \nu^{i} \rho\right) \rtimes \tau_{\text {temp }} .
\end{gathered}
$$

Proof. Theorem follows from an inductive application of Lemmas 4.8, $4.9,4.12,4.13,4.14$ and 4.15 , starting from $(\sigma, \widehat{\sigma})$-triple $(\emptyset, \sigma, \widehat{\sigma})$. We note that it follows from the definition of the $(\sigma, \widehat{\sigma})$-triple that in each step $\widehat{\sigma}$ is obtained as an irreducible subrepresentation of the induced representation having a unique irreducible subrepresentation.

First we are in the case of Lemma 4.8 (if $m=k+2$ ), or in the case of Lemma 4.12 (if $m \leq k+1$ ). Using a repeated application of these lemmas, together with Lemma 3.3 and a reasoning similar to the one used in [17, Section 3], we deduce that $\widehat{\sigma}$ is a subrepresentation of an induced representation of the form

$$
\prod_{i=1}^{k-m+2} \prod_{j=-a_{k-i+3}}^{-a_{k-i+2}-2} \delta\left(\left[\nu^{j-i+1} \rho, \nu^{j} \rho\right]\right) \times \prod_{j=-a_{m}}^{-a_{m-1}-1} \delta\left(\left[\nu^{j-k+m-2} \rho, \nu^{j} \rho\right]\right) \rtimes \pi_{1}^{\prime},
$$

for some irreducible representation $\pi_{1}^{\prime}$.

Note that, if $m \leq k$, after several applications of Lemma 4.12 we obtain that $\widehat{\sigma}$ is a subrepresentation of an induced representation of the form

$$
\prod_{i=1}^{k-m+2} \prod_{j=-a_{k-i+3}}^{-a_{k-i+2}-2} \delta\left(\left[\nu^{j-i+1} \rho, \nu^{j} \rho\right]\right) \rtimes \pi_{2}^{\prime},
$$


for some irreducible representation $\pi_{2}^{\prime}$. In that step appear induced representations

$$
\delta\left(\left[\nu^{-a_{m-1}} \rho, \nu^{a_{m}} \rho\right]\right) \rtimes S P\left(a_{1}, a_{2}, \ldots, a_{m-2}, a_{m}+1, \ldots, a_{m}+k-m+2\right),
$$

and

$\delta\left(\left[\nu^{-a_{m-2}} \rho, \nu^{a_{m-1}} \rho\right]\right) \rtimes S P\left(a_{1}, a_{2}, \ldots, a_{m-3}, a_{m}, a_{m}+1, \ldots, a_{m}+k-m+2\right)$.

Consequently, in the next application of Lemma $4.12, i_{\max }$ will be equal to $m$. Again, a repeated application of Lemma 4.12 produces the part

$$
\prod_{j=-a_{m}}^{-a_{m-1}-1} \delta\left(\left[\nu^{j-k+m-2} \rho, \nu^{j} \rho\right]\right)
$$

and in the last application of that lemma appear induced representations

$$
\begin{aligned}
& \delta\left(\left[\nu^{-a_{m-1}} \rho, \nu^{a_{m-1}} \rho\right]\right) \\
& \quad \rtimes S P\left(a_{1}, a_{2}, \ldots, a_{m-2}, a_{m-1}+1, \ldots, a_{m-1}+k-m+2\right),
\end{aligned}
$$

and

$$
\begin{aligned}
& \delta\left(\left[\nu^{-a_{m-2}} \rho, \nu^{a_{m-1}} \rho\right]\right) \\
& \quad \rtimes S P\left(a_{1}, a_{2}, \ldots, a_{m-3}, a_{m-1}, a_{m-1}+1, \ldots, a_{m-1}+k-m+2\right) .
\end{aligned}
$$

This brings us to a situation described either in Lemma 4.9 (if $m=k+2$ ), or in Lemma 4.13 (if $m \leq k+1$ ). A repeated application of an appropriate lemma produces the part $\prod_{j=-a_{m-1}}^{-a_{m-2}-1}\left(\delta\left(\left[\nu^{j-k+m-2} \rho, \nu^{j} \rho\right]\right) \times \nu^{j} \rho\right)$. In the last step we end with the induced representation

$$
\begin{aligned}
& \delta\left(\left[\nu^{-a_{m-2}} \rho, \nu^{a_{m-2}} \rho\right]\right) \\
& \quad \rtimes S P\left(a_{1}, a_{2}, \ldots, a_{m-3}, a_{m-2}, a_{m-2}+1, \ldots, a_{m-2}+k-m+2\right) .
\end{aligned}
$$

Hence, now we are either in a situation described in Lemma 4.14 (if $a_{m-2}+k-$ $m+2 \geq \alpha$ ) or in a situation described in Lemma 4.15 (if $a_{m-2}+k-m+2<\alpha$, i.e., $\left.a_{m-2}+k-m+2=\alpha-1\right)$.

If $a_{m-2}+k-m+2 \geq \alpha$, the strongly positive representation

$$
S P\left(a_{1}, a_{2}, \ldots, a_{m-3}, a_{m-2}, a_{m-2}+1, \ldots, a_{m-2}+k-m+2\right)
$$

is non-cuspidal, so we are in the case described in Lemma 4.14, and a repeated application of that lemma produces the part

$$
\prod_{i=k-m+3}^{k} \prod_{j=-a_{k-i+1}}^{-a_{k-i}-2}\left(\delta\left(\left[\nu^{j-i+1} \rho, \nu^{j} \rho\right]\right) \times \nu^{j-i+k-m+3} \rho \times \nu^{j-i+k-m+3} \rho\right) .
$$

Since

$$
\sigma_{\text {cusp }} \cong S P(-\lceil\alpha\rceil+\alpha,-\lceil\alpha\rceil+\alpha+1, \ldots, \alpha-1),
$$

the last application of Lemma 4.14 gives the induced representation

$$
\delta\left(\left[\nu^{-(\alpha-k+m-3)} \rho, \nu^{\alpha-k+m-3} \rho\right]\right) \rtimes \sigma_{\text {cusp }}
$$


if $m \neq 3$, and the representation $\tau_{\text {temp }}$ if $m=3$.

We note that in the case $a_{m-2}+k-m+2 \geq \alpha$ and $m=3$ we have either $r=k-\alpha$ or $r=-a_{1}$. If $r=-a_{1}$, then $a_{1}<a_{0}+2$ and, since $a_{1} \geq 0$ for $m=3$, we have $a_{1}=0$. Thus, if $a_{m-2}+k-m+2 \geq \alpha$ and $m=3$, then we have $r \in\left\{0, \frac{1}{2}\right\}$, so $r>\lceil\alpha\rceil-\alpha-1$ and the product $\prod_{i=r}^{\lceil\alpha\rceil-\alpha-1}\left(\nu^{i} \rho \times \nu^{i} \rho\right)$ is empty.

If $a_{m-2}+k-m+2 \geq \alpha$ and $m \neq 3$, using Lemma 4.15 we directly obtain the part $\prod_{i=r}^{\lceil\alpha\rceil-\alpha-1}\left(\nu^{i} \rho \times \nu^{i} \rho\right) \rtimes \tau_{\text {temp }}$.

It remains to consider the case $a_{m-2}+k-m+2<\alpha$. From a description of the strongly positive discrete series follows that $a_{m-2}+k-m+2=\alpha-1$, $a_{1}=\alpha-\lceil\alpha\rceil$ and $a_{i+1}=a_{i}+1$ for $i=0,1, \ldots, m-3$. Thus, the product

$$
\prod_{i=k-m+3}^{k} \prod_{j=-a_{k-i+1}}^{-a_{k-i}-2}\left(\delta\left(\left[\nu^{j-i+1} \rho, \nu^{j} \rho\right]\right) \times \nu^{j-i+k-m+3} \rho \times \nu^{j-i+k-m+3} \rho\right)
$$

is empty in this case, and $r=-a_{m-2}$.

The induced representation (4.8) is in this case isomorphic to the induced representation $\delta\left(\left[\nu^{r} \rho, \nu^{-r} \rho\right]\right) \rtimes \sigma_{\text {cusp }}$, and an application of Lemma 4.15 finishes the proof.

Note that, if we denote by $\sigma^{\prime}$ a discrete series subrepresentation of

$$
\delta\left(\left[\nu^{-a_{m-1}} \rho, \nu^{a_{m}} \rho\right]\right) \rtimes S P\left(a_{1}, a_{2}, \ldots, a_{m-2}, a_{m+1}, \ldots, a_{k+2}\right)
$$

non-isomorphic to $\sigma$, following the notation from the previous theorem, then it follows from Theorem 2.3 that either $m=k+2$ or $\sigma^{\prime}$ is a subrepresentation of

$$
\delta\left(\left[\nu^{-a_{m}} \rho, \nu^{a_{m+1}} \rho\right]\right) \rtimes S P\left(a_{1}, a_{2}, \ldots, a_{m-1}, a_{m+2}, \ldots, a_{k+2}\right) .
$$

In the first case, the Aubert dual of $\sigma^{\prime}$ is described in Theorem 4.11, while in the second case the Aubert dual of $\sigma^{\prime}$ can be obtained applying the previous theorem with indices one higher.

We also note that if $a_{m}-a_{m-1} \in\{1,2\}$ or $a_{m-1}-a_{m-2} \in\{1,2\}$, it follows from [5] that the unique irreducible subrepresentation of the induced representation (4.7) is unitarizable. Also, if $\sigma$ is a subquotient of a principal series, considered groups are split, and $\operatorname{char} F=0$, it follows from [25] that the unique irreducible subrepresentation of the induced representation (4.7) is unitarizable.

In the rest of this section we determine the Aubert dual of a discrete series $\sigma$ in the following two cases:

1. Discrete series $\sigma$ is a subrepresentation of an induced representation of the form $\delta\left(\left[\nu^{-a_{1}} \rho, \nu^{a_{2}} \rho\right]\right) \rtimes S P\left(a_{3}, a_{4}, \ldots, a_{k+2}\right)$ for $0 \leq a_{1}<$ $a_{2}<\cdots<a_{k}<a_{k+1}<a_{k+2}$ such that $\alpha-a_{i}$ is an integer for $i=1,2, \ldots, k+2$, and $\sigma$ is not a subrepresentation of $\delta\left(\left[\nu^{-a_{2}} \rho, \nu^{a_{3}} \rho\right]\right) \rtimes S P\left(a_{1}, a_{4}, \ldots, a_{k+2}\right)$. 
2. Discrete series $\sigma$ is a subrepresentation of an induced representation of the form $\delta\left(\left[\nu^{-a_{2}} \rho, \nu^{a_{3}} \rho\right]\right) \rtimes S P\left(a_{1}, a_{4}, \ldots, a_{k+2}\right)$ for $-\frac{1}{2}=a_{1}<$ $a_{2}<\cdots<a_{k}<a_{k+1}<a_{k+2}$ such that $\alpha-a_{i}$ is an integer for $i=1,2, \ldots, k+2, \alpha \geq \frac{3}{2}$, and $\sigma$ is not a subrepresentation of the induced representation $\delta\left(\left[\nu^{-a_{3}} \rho, \nu^{a_{4}} \rho\right]\right) \rtimes S P\left(a_{1}, a_{2}, a_{5} \ldots, a_{k+2}\right)$. A similar case when $\alpha=\frac{1}{2}$ will be considered in the following section.

We start our determination with several lemmas.

Lemma 4.17. Suppose that $\left(T, \pi, \pi^{\prime}\right)$ is a $(\sigma, \widehat{\sigma})$-triple such that $\pi$ is a subrepresentation of $\delta\left(\left[\nu^{-a_{1}} \rho, \nu^{a_{2}} \rho\right]\right) \rtimes S P\left(a_{3}, a_{4}, \ldots, a_{k+2}\right)$ for $0 \leq a_{1}<a_{2}<$ $\cdots<a_{k}<a_{k+1}<a_{k+2}$ such that $\alpha-a_{i}$ is an integer for $i=1,2, \ldots, k+2$, and $\pi$ is not a subrepresentation of $\delta\left(\left[\nu^{-a_{2}} \rho, \nu^{a_{3}} \rho\right]\right) \rtimes S P\left(a_{1}, a_{4}, \ldots, a_{k+2}\right)$. Let $l=|T|$. Let $i_{\max }$ denote the maximal $i$ such that the Jacquet module of $\pi$ with respect to an appropriate parabolic subgroup contains an irreducible constituent of the form $\nu^{a_{i}} \rho \otimes \nu^{a_{i+1}} \rho \otimes \cdots \otimes \nu^{a_{k+2}} \rho \otimes \sigma^{\prime}$. Let $x_{l+1}=a_{k+2}, y_{l+1}=a_{i_{\max }}$ and let $\pi_{1}^{\prime}$ denote an irreducible representation such that $\pi^{\prime} \hookrightarrow \delta\left(\left[\nu^{-a_{k+2}} \rho, \nu^{-a_{i \max }} \rho\right]\right) \rtimes \pi_{1}^{\prime}$. To define $\pi_{1}$, we consider several possibilities.

- If $i_{\max }>2$, let $\pi_{1}$ denote a discrete series subrepresentation of $\delta\left(\left[\nu^{-a_{1}} \rho, \nu^{a_{2}} \rho\right]\right) \rtimes S P\left(a_{3}, \ldots, a_{i_{\max }-1}, a_{i_{\max }}-1, \ldots, a_{k+2}-1\right)$,

which is not a subrepresentation of

$\delta\left(\left[\nu^{-a_{2}} \rho, \nu^{a_{3}} \rho\right]\right) \rtimes S P\left(a_{1}, a_{4}, \ldots, a_{i_{\max }-1}, a_{i_{\max }}-1, \ldots, a_{k+2}-1\right)$.

- If $i_{\max }=2$ and $a_{2}>a_{1}+1$, let $\pi_{1}$ denote a discrete series subrepresentation of $\delta\left(\left[\nu^{-a_{1}} \rho, \nu^{a_{2}-1} \rho\right]\right) \rtimes S P\left(a_{3}-1, \ldots, a_{k+2}-1\right)$, which is not a subrepresentation of $\delta\left(\left[\nu^{-a_{2}+1} \rho, \nu^{a_{3}-1} \rho\right]\right) \rtimes S P\left(a_{1}, a_{4}-1, \ldots, a_{k+2}-1\right)$.

- If $i_{\max }=2$ and $a_{2}=a_{1}+1$, let $\pi_{1}$ denote a tempered subrepresentation of $\delta\left(\left[\nu^{-a_{1}} \rho, \nu^{a_{1}} \rho\right]\right) \rtimes S P\left(a_{3}-1, \ldots, a_{k+2}-1\right)$ which does not contain an irreducible constituent of the form $\nu^{a_{3}-1} \rho \otimes \sigma^{\prime}$ in the Jacquet module with respect to an appropriate parabolic subgroup.

Then $\left(T \cup\left\{\left(x_{l+1}, y_{l+1}\right)\right\}, \pi_{1}, \pi_{1}^{\prime}\right)$ is a $(\sigma, \widehat{\sigma})$-triple.

Proof. This lemma can be proved in the same way as Lemma 4.12. Let us just comment on the definition of $\pi_{1}$ in the case $i_{\max }=2$ and $a_{2}=a_{1}+1$. Since in this case $a_{i+1}=a_{i}+1$ for $i=2,3, \ldots, k+1$, it follows that if an irreducible constituent of the form $\nu^{a_{3}-1} \rho \otimes \sigma^{\prime}$ appears in $\mu^{*}\left(\delta\left(\left[\nu^{-a_{1}} \rho, \nu^{a_{1}} \rho\right]\right) \rtimes\right.$ $\left.S P\left(a_{3}-1, \ldots, a_{k+2}-1\right)\right)$, then $\sigma^{\prime} \cong \delta\left(\left[\nu^{-a_{1}} \rho, \nu^{a_{1}} \rho\right]\right) \rtimes S P\left(a_{3}-2, a_{4}-\right.$ $\left.1, \ldots, a_{k+2}-1\right)$, and such constituent appears in $\mu^{*}\left(\delta\left(\left[\nu^{-a_{1}} \rho, \nu^{a_{1}} \rho\right]\right) \rtimes S P\left(a_{3}-\right.\right.$ $\left.\left.1, \ldots, a_{k+2}-1\right)\right)$ with multiplicity one. Thus, there is a unique irreducible subrepresentation of $\delta\left(\left[\nu^{-a_{1}} \rho, \nu^{a_{1}} \rho\right]\right) \rtimes S P\left(a_{3}-1, \ldots, a_{k+2}-1\right)$ which contains an irreducible constituent of the form $\nu^{a_{3}-1} \rho \otimes \sigma^{\prime}$ in the Jacquet module with respect to an appropriate parabolic subgroup. 
Suppose, on the contrary, that $\mu^{*}\left(\pi_{1}\right) \geq \nu^{a_{2}} \rho \otimes \sigma^{\prime}$. Then the Jacquet module of $\pi$ with respect to an appropriate parabolic subgroup contains an irreducible constituent of the form $\nu^{a_{2}} \rho \otimes \nu^{a_{3}} \rho \otimes \cdots \otimes \nu^{a_{k+2}} \rho \otimes \nu^{a_{2}} \rho \otimes \sigma_{1}^{\prime}$. This implies that there is a representation $\sigma_{2}^{\prime}$ such that $\pi$ is a subrepresentation of $\nu^{a_{2}} \rho \times \nu^{a_{3}} \rho \times \cdots \times \nu^{a_{k+2}} \rho \times \nu^{a_{2}} \rho \rtimes \sigma_{2}^{\prime}$. Since $i_{\max }=2$, we obtain

$$
\pi \hookrightarrow L\left(\nu^{a_{2}} \rho, \nu^{a_{3}} \rho, \ldots, \nu^{a_{k+2}} \rho\right) \times \nu^{a_{2}} \rho \rtimes \sigma_{2}^{\prime},
$$

and Frobenius reciprocity, together with transitivity of Jacquet modules and Lemma 3.1, implies that $\mu^{*}(\pi) \geq \nu^{a_{2}} \rho \times \nu^{a_{2}} \rho \otimes \sigma_{3}^{\prime}$, for an irreducible representation $\sigma_{3}^{\prime}$, which is impossible.

Next lemma can be proved in the same way as the previous one.

Lemma 4.18. Suppose that $\left(T, \pi, \pi^{\prime}\right)$ is a $(\sigma, \widehat{\sigma})$-triple such that $\pi$ is a subrepresentation of $\delta\left(\left[\nu^{-a_{2}} \rho, \nu^{a_{3}} \rho\right]\right) \rtimes S P\left(a_{1}, a_{4}, \ldots, a_{k+2}\right)$ for $-\frac{1}{2}=a_{1}<a_{2}<$ $\cdots<a_{k}<a_{k+1}<a_{k+2}$ such that $\alpha-a_{i}$ is an integer for $i=1,2, \ldots, k+2$, and $\pi$ is not a subrepresentation of $\delta\left(\left[\nu^{-a_{3}} \rho, \nu^{a_{4}} \rho\right]\right) \rtimes S P\left(a_{1}, a_{2}, a_{5} \ldots, a_{k+2}\right)$. Let $l=|T|$. Let $i_{\max }$ denote the maximal $i$ such that the Jacquet module of $\pi$ with respect to an appropriate parabolic subgroup contains an irreducible constituent of the form $\nu^{a_{i}} \rho \otimes \nu^{a_{i+1}} \rho \otimes \cdots \otimes \nu^{a_{k+2}} \rho \otimes \sigma^{\prime}$. Let $x_{l+1}=a_{k+2}, y_{l+1}=a_{i_{\max }}$ and let $\pi_{1}^{\prime}$ denote an irreducible representation

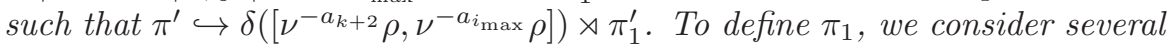
possibilities.

- If $i_{\max }>3$, let $\pi_{1}$ denote a discrete series subrepresentation of $\delta\left(\left[\nu^{-a_{2}} \rho, \nu^{a_{3}} \rho\right]\right) \rtimes S P\left(a_{1}, a_{4}, \ldots, a_{i_{\max }-1}, a_{i_{\max }}-1, \ldots, a_{k+2}-1\right)$, which is not a subrepresentation of $\delta\left(\left[\nu^{-a_{3}} \rho, \nu^{a_{4}} \rho\right]\right) \rtimes S P\left(a_{1}, a_{5}, \ldots, a_{i_{\max }-1}, a_{i_{\max }}-1, \ldots, a_{k+2}-1\right)$.

- If $i_{\max }=3$ and $a_{3}>a_{2}+1$, let $\pi_{1}$ denote a discrete series subrepresentation of $\delta\left(\left[\nu^{-a_{2}} \rho, \nu^{a_{3}-1} \rho\right]\right) \rtimes S P\left(a_{1}, a_{4}-1, \ldots, a_{k+2}-1\right)$, which is not a subrepresentation of $\delta\left(\left[\nu^{-a_{3}+1} \rho, \nu^{a_{4}-1} \rho\right]\right) \rtimes S P\left(a_{1}, a_{5}-1, \ldots, a_{k+2}-1\right)$.

- If $i_{\max }=3$ and $a_{3}=a_{2}+1$, let $\pi_{1}$ denote a tempered subrepresentation of $\delta\left(\left[\nu^{-a_{2}} \rho, \nu^{a_{2}} \rho\right]\right) \rtimes S P\left(a_{1}, a_{4}-1, \ldots, a_{k+2}-1\right)$, which does not contain an irreducible constituent of the form $\nu^{a_{4}-1} \rho \otimes \sigma^{\prime}$ in the Jacquet module with respect to an appropriate parabolic subgroup.

Then $\left(T \cup\left\{\left(x_{l+1}, y_{l+1}\right)\right\}, \pi_{1}, \pi_{1}^{\prime}\right)$ is a $(\sigma, \widehat{\sigma})$-triple.

The following two lemmas enable us to handle a tempered case which appears in an application of the inductive procedure.

Lemma 4.19. Suppose that $\left(T, \pi, \pi^{\prime}\right)$ is a $(\sigma, \widehat{\sigma})$-triple such that $\pi$ is a subrepresentation of $\delta\left(\left[\nu^{-a} \rho, \nu^{a} \rho\right]\right) \rtimes S P(a+1, \ldots, a+k)$ and $\mu^{*}(\pi)$ does not contain an irreducible constituent of the form $\nu^{a+1} \rho \otimes \sigma^{\prime}$. Let $l=|T|$. 
- If $a>\lceil\alpha\rceil-\alpha$, let $x_{l+1}=a+k, y_{l+1}=a, x_{l+2}=y_{l+2}=a$ and let $\pi_{1}^{\prime}$ denote an irreducible representation such that $\pi^{\prime} \hookrightarrow \delta\left(\left[\nu^{-a-k} \rho, \nu^{-a} \rho\right]\right) \times$ $\nu^{-a} \rho \rtimes \pi_{1}^{\prime}$. Also, let $\pi_{1}$ denote a tempered subrepresentation of

$$
\delta\left(\left[\nu^{-a+1} \rho, \nu^{a-1} \rho\right]\right) \rtimes S P(a, \ldots, a+k-1)
$$

which does not contain an irreducible constituent of the form $\nu^{a} \rho \otimes \sigma^{\prime}$ in the Jacquet module with respect to an appropriate parabolic subgroup. Then $\left(T \cup\left\{\left(x_{l+1}, y_{l+1}\right),\left(x_{l+2}, y_{l+2}\right)\right\}, \pi_{1}, \pi_{1}^{\prime}\right)$ is a $(\sigma, \widehat{\sigma})$-triple.

- If $a=0$, then $\pi^{\prime}$ is the unique irreducible subrepresentation of the induced representation $\delta\left(\left[\nu^{-\alpha} \rho, \rho\right]\right) \rtimes \sigma_{\text {cusp }}$.

- If $a=\frac{1}{2}$, then $\pi^{\prime}$ is the unique irreducible subrepresentation of the induced representation $\delta\left(\left[\nu^{-\alpha-1} \rho, \nu^{-\frac{1}{2}} \rho\right]\right) \rtimes \pi_{1}^{\prime}$, where

(i) $\pi_{1}^{\prime} \cong L\left(\delta\left(\left[\nu^{-\alpha} \rho, \nu^{\frac{1}{2}} \rho\right]\right), \sigma_{\text {cusp }}\right)$, if $\alpha>\frac{1}{2}$, and

(ii) $\pi_{1}^{\prime}$ is a tempered subrepresentation of $\delta\left(\left[\nu^{-\frac{1}{2}} \rho, \nu^{\frac{1}{2}} \rho\right]\right) \rtimes \sigma_{\text {cusp }}$ which is not a subrepresentation of $\nu^{\frac{1}{2}} \rho \rtimes S P\left(\frac{1}{2}\right)$, if $\alpha=\frac{1}{2}$.

Proof. The case $a>\lceil\alpha\rceil-\alpha$ can be handled following the same lines as in the proofs of Lemma 4.13 and Lemma 4.17.

Now we deal with the case $a=0$. Note that if $\mu^{*}\left(\pi^{\prime}\right) \geq \nu^{x} \rho \otimes \sigma^{\prime}$, for some irreducible $\sigma^{\prime}$, then $x=0$. Also, $\pi$ is a subrepresentation of $\rho \times$ $\nu \rho \times \cdots \times \nu^{\alpha} \rho \rtimes \sigma_{\text {cusp. }}$. In the same way as before, we conclude that $\pi^{\prime}$ is a subrepresentation of $\rho \times \nu^{-1} \rho \times \cdots \times \nu^{-\alpha} \rho \rtimes \sigma_{\text {cusp }}$ and Lemma 3.4 implies $\pi^{\prime} \cong L\left(\delta\left(\left[\nu^{-\alpha} \rho, \rho\right]\right), \sigma_{\text {cusp }}\right)$.

It remains to consider the case $a=\frac{1}{2}$. Let us first show that in this case the Jacquet module of $\pi$ with respect to an appropriate parabolic subgroup does not contain an irreducible representation of the form $\nu^{\frac{1}{2}} \rho \otimes \nu^{\frac{1}{2}} \rho \otimes \sigma^{\prime}$ or, equivalently, as one can see directly from the structural formula, $\mu^{*}(\pi)$ does not contain the irreducible constituent $\nu^{\frac{1}{2}} \rho \times \nu^{\frac{1}{2}} \rho \otimes S P\left(\frac{3}{2}, \ldots, \alpha+1\right)$. Suppose, on the contrary, that $\mu^{*}(\pi) \geq \nu^{\frac{1}{2}} \rho \times \nu^{\frac{1}{2}} \rho \otimes S P\left(\frac{3}{2}, \ldots, \alpha+1\right)$. Then the Jacquet module of $\pi$ with respect to an appropriate parabolic subgroup contains

$$
\nu^{\frac{1}{2}} \rho \times \nu^{\frac{1}{2}} \rho \otimes \delta\left(\left[\nu^{\frac{1}{2}} \rho, \nu^{\frac{3}{2}} \rho\right]\right) \otimes S P\left(-\frac{1}{2}, \frac{5}{2}, \ldots, \alpha+1\right) .
$$

Transitivity of Jacquet modules implies that there is a representation $\delta \in$ $\operatorname{Irr}\left(G L\left(n_{\delta}, F\right)\right)$ such that $\mu^{*}(\pi) \geq \delta \otimes S P\left(-\frac{1}{2}, \frac{5}{2}, \ldots, \alpha+1\right)$ and $m^{*}(\delta) \geq$ $\nu^{\frac{1}{2}} \rho \times \nu^{\frac{1}{2}} \rho \otimes \delta\left(\left[\nu^{\frac{1}{2}} \rho, \nu^{\frac{3}{2}} \rho\right]\right)$. An application of the structural formula shows that $\delta \cong \nu^{\frac{1}{2}} \rho \times \nu^{\frac{1}{2}} \rho \times \delta\left(\left[\nu^{\frac{1}{2}} \rho, \nu^{\frac{3}{2}} \rho\right]\right)$, which leads to $\mu^{*}(\pi) \geq \nu^{\frac{3}{2}} \rho \otimes \sigma^{\prime}$, for some irreducible $\sigma^{\prime}$, a contradiction.

As in $[12$, Section 4], we obtain that $S P(a+1, \ldots, a+k)$ is a subrepresentation of $\nu^{a+1} \rho \times \cdots \times \nu^{a+k} \rho \rtimes S P(a, \ldots, a+k-1)$, so $\pi$ is a subrepresentation of

$$
\nu^{\frac{1}{2}} \rho \times \nu^{\frac{3}{2}} \rho \times \cdots \times \nu^{\alpha+1} \rho \times \nu^{-\frac{1}{2}} \rho \rtimes S P\left(\frac{1}{2}, \ldots, \alpha\right) .
$$


From the structural formula, [14, Theorem 4.6] and the fact that $\mu^{*}(\pi)$ does not contain an irreducible constituent of the form $\nu^{a+1} \rho \otimes \sigma^{\prime}$, we deduce that if $\mu^{*}(\pi) \geq \nu^{x} \rho \otimes \sigma^{\prime}$, then $x=\frac{1}{2}$. Corollary 4.7 implies that there is an irreducible representation $\tau$ such that $\pi^{\prime}$ is a subrepresentation of $\delta\left(\left[\nu^{-\alpha-1} \rho, \nu^{-\frac{1}{2}} \rho\right]\right) \rtimes \tau$. Also, if $\mu^{*}\left(\pi^{\prime}\right) \geq \nu^{x} \rho \otimes \sigma^{\prime}$, for some irreducible $\sigma^{\prime}$, then $x=-\frac{1}{2}$. Furthermore, if $\mu^{*}\left(\pi^{\prime}\right) \geq \nu^{-\frac{1}{2}} \rho \otimes \sigma^{\prime}$, then $\mu^{*}\left(\sigma^{\prime}\right)$ does not contain an irreducible constituent of the form $\nu^{-\frac{1}{2}} \rho \otimes \sigma^{\prime \prime}$. This also implies that if $\mu^{*}(\tau) \geq \nu^{x} \rho \otimes \sigma^{\prime}$, then $x=\frac{1}{2}$, since otherwise we would have embeddings $\tau \hookrightarrow \nu^{x} \rho \rtimes \tau^{\prime}$ and $\pi^{\prime} \hookrightarrow$ $\nu^{x} \rho \times \nu^{-\frac{1}{2}} \rho \times \delta\left(\left[\nu^{-\alpha-1} \rho, \nu^{-\frac{3}{2}} \rho\right]\right) \rtimes \tau^{\prime}$, for some irreducible representation $\tau^{\prime}$, which is impossible.

Since $\nu^{\frac{1}{2}} \rho$ appears twice in the cuspidal support of $\tau$, the representation $\tau$ is not strongly positive. Also, $\tau$ is not a discrete series representation, since otherwise we would have an embedding $\tau \hookrightarrow \delta\left(\left[\nu^{-y} \rho, \nu^{x} \rho\right]\right) \rtimes \tau^{\prime}$, for $x>y \geq \frac{1}{2}$ and $\tau^{\prime}$ irreducible, a contradiction.

Let us consider two possibilities, depending of $\alpha$.

First, we assume that $\alpha=\frac{1}{2}$. From the cuspidal support of $\tau$ and the fact that $\mu^{*}\left(\pi^{\prime}\right)$ does not contain an irreducible constituent of the form $\nu^{-\frac{1}{2}} \rho \otimes$ $\nu^{-\frac{1}{2}} \rho \otimes \sigma^{\prime}$, we see that $\tau$ is an irreducible subrepresentation of $\delta\left(\left[\nu^{-\frac{1}{2}} \rho, \nu^{\frac{1}{2}} \rho\right]\right) \rtimes$ $\sigma_{\text {cusp }}$. Let us show that $\tau$ is not a subrepresentation of $\nu^{\frac{1}{2}} \rho \rtimes S P\left(\frac{1}{2}\right)$. Suppose, on the contrary, that $\tau$ is a subrepresentation of $\nu^{\frac{1}{2}} \rho \rtimes S P\left(\frac{1}{2}\right)$. Then $\mu^{*}(\tau)$ contains $\nu^{\frac{1}{2}} \rho \times \nu^{\frac{1}{2}} \rho \otimes \sigma_{\text {cusp }}$, which provides an embedding

$$
\pi^{\prime} \hookrightarrow \delta\left(\left[\nu^{-\alpha-1} \rho, \nu^{-\frac{1}{2}} \rho\right]\right) \times \nu^{\frac{1}{2}} \rho \times \nu^{\frac{1}{2}} \rho \rtimes \sigma_{\text {cusp }}
$$

Clearly, $\pi^{\prime}$ is then a subrepresentation of the induced representation

$$
L\left(\delta\left(\left[\nu^{-\alpha-1} \rho, \nu^{-\frac{1}{2}} \rho\right]\right), \nu^{\frac{1}{2}} \rho\right) \times \nu^{\frac{1}{2}} \rho \rtimes \sigma_{\text {cusp }},
$$

but then Lemma 3.1 gives

$$
\pi^{\prime} \hookrightarrow \nu^{\frac{1}{2}} \rho \times L\left(\delta\left(\left[\nu^{-\alpha-1} \rho, \nu^{-\frac{1}{2}} \rho\right]\right), \nu^{\frac{1}{2}} \rho\right) \rtimes \sigma_{\text {cusp }},
$$

a contradiction.

Now we assume that $\alpha>\frac{1}{2}$. Let $\tau \cong L\left(\delta_{1}, \delta_{2}, \ldots, \delta_{m}, \tau_{\text {temp }}\right)$. Suppose that $\tau \cong \tau_{\text {temp }}$. Then $\tau$ is a subrepresentation of an induced representation of the form $\delta\left(\left[\nu^{-x} \rho, \nu^{x} \rho\right]\right) \rtimes \tau^{\prime}$, for $\tau^{\prime}$ irreducible tempered. Clearly, $x=$ $\frac{1}{2}$ and from the cuspidal support of $\tau$ we see that $\tau^{\prime}$ is a strongly positive representation. Thus, by $\left[14\right.$, Lemma 3.6], $\tau^{\prime} \cong S P\left(-\frac{1}{2}, \frac{3}{2}, \ldots, \alpha\right)$. This gives us the following embeddings and isomorphism:

$$
\begin{aligned}
\pi^{\prime} & \hookrightarrow \delta\left(\left[\nu^{-\alpha-1} \rho, \nu^{-\frac{1}{2}} \rho\right]\right) \times \delta\left(\left[\nu^{-\frac{1}{2}} \rho, \nu^{\frac{1}{2}} \rho\right]\right) \rtimes S P\left(-\frac{1}{2}, \frac{3}{2}, \ldots, \alpha\right) \\
& \hookrightarrow \nu^{-\frac{1}{2}} \rho \times \delta\left(\left[\nu^{-\alpha-1} \rho, \nu^{-\frac{3}{2}} \rho\right]\right) \times \nu^{\frac{1}{2}} \rho \times \nu^{-\frac{1}{2}} \rho \times \nu^{\frac{3}{2}} \rho \rtimes S P\left(-\frac{1}{2}, \frac{1}{2}, \ldots, \alpha\right) \\
& \cong \nu^{-\frac{1}{2}} \rho \times \nu^{\frac{1}{2}} \rho \times \nu^{\frac{3}{2}} \rho \times \delta\left(\left[\nu^{-\alpha-1} \rho, \nu^{-\frac{3}{2}} \rho\right]\right) \times \nu^{-\frac{1}{2}} \rho \rtimes S P\left(-\frac{1}{2}, \frac{1}{2}, \ldots, \alpha\right) .
\end{aligned}
$$


It follows that the Jacquet module of $\pi^{\prime}$ with respect to an appropriate parabolic subgroup contains an irreducible constituent of the form $\nu^{-\frac{1}{2}} \rho \otimes$ $\nu^{\frac{1}{2}} \rho \otimes \nu^{\frac{3}{2}} \rho \otimes \sigma_{1}$. Thus, the Jacquet module of $\pi$ with respect to an appropriate parabolic subgroup contains an irreducible constituent of the form $\nu^{\frac{1}{2}} \rho \otimes \nu^{-\frac{1}{2}} \rho \otimes \nu^{-\frac{3}{2}} \rho \otimes \sigma_{2}$, contradicting the temperedness of $\pi$.

Thus, $\tau$ is non-tempered and $m \geq 1$. Let $\delta_{i}=\delta\left(\left[\nu^{x_{i}} \rho, \nu^{y_{i}} \rho\right]\right)$, for $i=$ $1,2, \ldots, m$. It follows at once that $y_{1}=\frac{1}{2}$ and $x_{1} \leq-\frac{3}{2}$. If $m \geq 2$, from the cuspidal support of $\tau$ we see that $y_{2} \leq x_{1}-1$, contradicting $e\left(\delta_{1}\right) \leq e\left(\delta_{2}\right)$. Thus, $m=1$. Also, $\tau_{\text {temp }}$ is a strongly positive representation and it is isomorphic to $S P\left(-\frac{1}{2}, \frac{1}{2}, \ldots,-x_{1}-1,-x_{1}+1,-x_{1}+2, \ldots, \alpha\right)$. If $x_{1}<-\alpha$, $\tau_{\text {temp }}$ is a subrepresentation of $\nu^{-x_{1}+1} \rho \rtimes \sigma_{s p}$, for an appropriate strongly positive representation $\sigma_{s p}$. Since $-x_{1}+1 \geq \frac{5}{2}$, this provides the following embedding and isomorphism:

$$
\begin{aligned}
\pi^{\prime} & \hookrightarrow \delta\left(\left[\nu^{-\alpha-1} \rho, \nu^{-\frac{1}{2}} \rho\right]\right) \times \delta\left(\left[\nu^{x_{1}} \rho, \nu^{\frac{1}{2}} \rho\right]\right) \times \nu^{-x_{1}+1} \rho \rtimes \sigma_{s p} \\
& \cong \nu^{-x_{1}+1} \rho \times \delta\left(\left[\nu^{-\alpha-1} \rho, \nu^{-\frac{1}{2}} \rho\right]\right) \times \delta\left(\left[\nu^{x_{1}} \rho, \nu^{\frac{1}{2}} \rho\right]\right) \rtimes \sigma_{s p} .
\end{aligned}
$$

This implies that $\mu^{*}\left(\pi^{\prime}\right) \geq \nu^{-x_{1}+1} \rho \otimes \sigma^{\prime}$, for some irreducible $\sigma^{\prime}$, which is impossible. Consequently, $x_{1}=-\alpha$ and in this case $\tau$ is a unique irreducible (Langlands) subrepresentation of $\delta\left(\left[\nu^{-\alpha} \rho, \nu^{\frac{1}{2}} \rho\right]\right) \rtimes \sigma_{\text {cusp. }}$. This finishes the proof.

Lemma 4.20. Suppose that $\left(T, \pi, \pi^{\prime}\right)$ is a $(\sigma, \widehat{\sigma})$-triple such that $\pi$ is a subrepresentation of $\delta\left(\left[\nu^{-a} \rho, \nu^{a} \rho\right]\right) \rtimes S P\left(-\frac{1}{2}, a+1, \ldots, a+k-1\right)$ which does not contain an irreducible constituent of the form $\nu^{a+1} \rho \otimes \sigma^{\prime}$ in the Jacquet module with respect to an appropriate parabolic subgroup. Let $l=|T|$.

- If $a>\frac{1}{2}$, let $x_{l+1}=a+k-1, y_{l+1}=a, x_{l+2}=y_{l+2}=a$ and let $\pi_{1}^{\prime}$ denote an irreducible representation such that $\pi^{\prime} \hookrightarrow$ $\delta\left(\left[\nu^{-a-k+1} \rho, \nu^{-a} \rho\right]\right) \times \nu^{-a} \rho \rtimes \pi_{1}^{\prime}$. Also, let $\pi_{1}$ denote a tempered subrepresentation of

$$
\delta\left(\left[\nu^{-a+1} \rho, \nu^{a-1} \rho\right]\right) \rtimes S P\left(-\frac{1}{2}, a, \ldots, a+k-2\right)
$$

which does not contain an irreducible constituent of the form $\nu^{a} \rho \otimes \sigma^{\prime}$ in the Jacquet module with respect to an appropriate parabolic subgroup. Then $\left(T \cup\left\{\left(x_{l+1}, y_{l+1}\right),\left(x_{l+2}, y_{l+2}\right)\right\}, \pi_{1}, \pi_{1}^{\prime}\right)$ is a $(\sigma, \widehat{\sigma})$-triple.

- If $a=\frac{1}{2}$, then $\pi^{\prime}$ is the unique irreducible subrepresentation of the induced representation $\delta\left(\left[\nu^{-\alpha} \rho, \nu^{-\frac{1}{2}} \rho\right]\right) \times \nu^{-\frac{1}{2}} \rho \rtimes \sigma_{\text {cusp. }}$.

Proof. We discuss only the case $a=\frac{1}{2}$, since the other case can be handled in the same way as in the proof of Lemmas 4.13 and 4.17. We have 
the following embeddings and isomorphism:

$$
\begin{aligned}
\pi & \hookrightarrow \delta\left(\left[\nu^{-\frac{1}{2}} \rho, \nu^{\frac{1}{2}} \rho\right]\right) \rtimes S P\left(-\frac{1}{2}, \frac{3}{2}, \ldots, \alpha\right) \\
& \hookrightarrow \nu^{\frac{1}{2}} \rho \times \nu^{-\frac{1}{2}} \rho \times \nu^{\frac{3}{2}} \rho \times \cdots \times \nu^{\alpha} \rho \rtimes \sigma_{\text {cusp }} \\
& \simeq \nu^{\frac{1}{2}} \rho \times \nu^{\frac{3}{2}} \rho \times \cdots \times \nu^{\alpha} \rho \times \nu^{-\frac{1}{2}} \rho \rtimes \sigma_{\text {cusp }}
\end{aligned}
$$

Frobenius reciprocity implies that the Jacquet module of $\pi$ with respect to an appropriate parabolic subgroup contains $\nu^{\frac{1}{2}} \rho \otimes \nu^{\frac{3}{2}} \rho \otimes \cdots \otimes \nu^{\alpha} \rho \otimes \nu^{-\frac{1}{2}} \rho \otimes$ $\sigma_{\text {cusp }}$. Since $\mu^{*}(\pi)$ does not contain an irreducible constituent of the form $\nu^{x} \rho \otimes \sigma^{\prime}$ for $x \geq \frac{3}{2}$, in the same way as in the proof of Lemma 4.6 one can see that $\pi^{\prime}$ is a subrepresentation of an induced representation of the form $\delta\left(\left[\nu^{-\alpha} \rho, \nu^{-\frac{1}{2}} \rho\right]\right) \rtimes \sigma^{\prime \prime}$, for irreducible $\sigma^{\prime \prime}$.

Note that the cuspidal support of $\sigma^{\prime \prime}$ consists of $\nu^{\frac{1}{2}} \rho$ and $\sigma_{\text {cusp. }}$. Since the induced representation $\nu^{\frac{1}{2}} \rho \rtimes \sigma_{\text {cusp }}$ is irreducible, it follows that $\sigma^{\prime \prime} \cong$ $\nu^{-\frac{1}{2}} \rho \rtimes \sigma_{\text {cusp }}$, and lemma is proved.

The following theorem can be proved in the same way as Theorem 4.16, using a repeated application of Lemma 4.17 and Lemma 4.19.

THEOREM 4.21. Suppose that a discrete series $\sigma$ is a subrepresentation of $\delta\left(\left[\nu^{-a_{1}} \rho, \nu^{a_{2}} \rho\right]\right) \rtimes S P\left(a_{3}, a_{4}, \ldots, a_{k+2}\right)$ for $0 \leq a_{1}<a_{2}<\cdots<a_{k}<a_{k+1}<$ $a_{k+2}$ such that $\alpha-a_{i}$ is an integer for $i=1,2, \ldots, k+2$, and that $\sigma$ is not a subrepresentation of $\delta\left(\left[\nu^{-a_{2}} \rho, \nu^{a_{3}} \rho\right]\right) \rtimes S P\left(a_{1}, a_{4}, \ldots, a_{k+2}\right)$. Let $a_{0}=\lceil\alpha\rceil-\alpha$.

The Aubert dual $\widehat{\sigma}$ of $\sigma$ is the unique irreducible subrepresentation of the induced representation

$$
\begin{aligned}
\prod_{i=1}^{k} & \prod_{j=-a_{k-i+3}}^{-a_{k-i+2}-2} \delta\left(\left[\nu^{j-i+1} \rho, \nu^{j} \rho\right]\right) \times \prod_{j=-a_{2}}^{-a_{1}-1} \delta\left(\left[\nu^{j-k} \rho, \nu^{j} \rho\right]\right) \times \\
& \times \prod_{j=-a_{1}}^{-a_{0}-1}\left(\delta\left(\left[\nu^{j-k} \rho, \nu^{j} \rho\right]\right) \times \nu^{j} \rho\right) \rtimes \pi,
\end{aligned}
$$

where $\pi$ stands for

- $L\left(\delta\left(\left[\nu^{-\alpha} \rho, \rho\right]\right), \sigma_{\text {cusp }}\right)$, if $\alpha=\lceil\alpha\rceil$,

- $L\left(\delta\left(\left[\nu^{-\alpha-1} \rho, \nu^{-\frac{1}{2}} \rho\right]\right), \delta\left(\left[\nu^{-\alpha} \rho, \nu^{\frac{1}{2}} \rho\right]\right), \sigma_{\text {cusp }}\right)$, if $\alpha \neq\lceil\alpha\rceil$ and $\alpha \geq \frac{3}{2}$,

- $L\left(\delta\left(\left[\nu^{-\alpha-1} \rho, \nu^{-\frac{1}{2}} \rho\right]\right), \tau_{\text {temp }}\right)$, where $\tau_{\text {temp }}$ is a tempered subrepresentation of $\delta\left(\left[\nu^{-\frac{1}{2}} \rho, \nu^{\frac{1}{2}} \rho\right]\right) \rtimes \sigma_{\text {cusp }}$ which is not a subrepresentation of $\nu^{\frac{1}{2}} \rho \rtimes S P\left(\frac{1}{2}\right)$, if $\alpha=\frac{1}{2}$.

We also note that if $a_{2}-a_{1} \in\{1,2\}$ then it follows from [5] that the unique irreducible subrepresentation of the induced representation (4.9) is unitarizable. Also, if $\sigma$ is a subquotient of a principal series, considered groups are split, and $\operatorname{char} F=0$, it follows from [25] that the unique irreducible subrepresentation of the induced representation (4.9) is unitarizable. 
The following theorem can also be proved in the same way as Theorem 4.16, using a repeated application of Lemma 4.18 and Lemma 4.20.

THEOREM 4.22. Suppose that a discrete series $\sigma$ is a subrepresentation of $\delta\left(\left[\nu^{-a_{2}} \rho, \nu^{a_{3}} \rho\right]\right) \rtimes S P\left(a_{1}, a_{4}, \ldots, a_{k+2}\right)$ for $-\frac{1}{2}=a_{1}<a_{2}<\cdots<a_{k}<$ $a_{k+1}<a_{k+2}$ such that $\alpha-a_{i}$ is an integer for $i=1,2, \ldots, k+2$, and that $\sigma$ is not a subrepresentation of $\delta\left(\left[\nu^{-a_{3}} \rho, \nu^{a_{4}} \rho\right]\right) \rtimes S P\left(a_{1}, a_{2}, a_{5} \ldots, a_{k+2}\right)$. The Aubert dual $\hat{\sigma}$ of $\sigma$ is the unique irreducible subrepresentation of the induced representation of the form

$$
\begin{aligned}
& \prod_{i=1}^{k-1} \prod_{j=-a_{k-i+3}}^{-a_{k-i+2}-2} \delta\left(\left[\nu^{j-i+1} \rho, \nu^{j} \rho\right]\right) \times \prod_{j=-a_{3}}^{-a_{2}-1} \delta\left(\left[\nu^{j-k+1} \rho, \nu^{j} \rho\right]\right) \times \\
& \times \prod_{j=-a_{2}}^{-\frac{3}{2}}\left(\delta\left(\left[\nu^{j-k+1} \rho, \nu^{j} \rho\right]\right) \times \nu^{j} \rho\right) \times \delta\left(\left[\nu^{-\alpha} \rho, \nu^{-\frac{1}{2}} \rho\right]\right) \times \nu^{-\frac{1}{2}} \rho \rtimes \sigma_{\text {cusp }} .
\end{aligned}
$$

We also note that if $a_{3}-a_{2} \in\{1,2\}$ then it follows from [5] that the unique irreducible subrepresentation of the induced representation (4.10) is unitarizable. Also, if $\sigma$ is a subquotient of a principal series, considered groups are split, and $\operatorname{char} F=0$, it follows from [25] that the unique irreducible subrepresentation of the induced representation (4.10) is unitarizable.

\section{TWO EXCEPTIONAL CASES}

In this section we discuss the remaining two cases. First, we deal with the case when the rank one reducibility equals zero.

TheOREM 5.1. Suppose that $\rho \rtimes \sigma_{\text {cusp }}$ reduces and write $\rho \rtimes \sigma_{\text {cusp }}=$ $\tau_{1}+\tau_{-1}$ as a sum of mutually non-isomorphic tempered representations in $R(G)$. Let $a, b$ denote non-negative integers such that $a<b$ and let $\sigma_{i}$ denote a discrete series subrepresentation of $\delta\left(\left[\nu^{-a} \rho, \nu^{b} \rho\right]\right) \rtimes \sigma_{\text {cusp }}$ such that $\sigma_{i}$ is a subrepresentation of $\delta\left(\left[\nu \rho, \nu^{a} \rho\right]\right) \times \delta\left(\left[\nu \rho, \nu^{b} \rho\right]\right) \rtimes \tau_{i}$, for $i=1,-1$. The Aubert dual $\widehat{\sigma}_{i}$ of $\sigma_{i}$ is the unique irreducible subrepresentation of the induced representation

$$
\begin{aligned}
\nu^{-b} \rho & \times \nu^{-b+1} \rho \times \cdots \times \nu^{-a-1} \rho \times \nu^{-a} \rho \times \nu^{-a} \rho \times \nu^{-a+1} \rho \\
& \times \nu^{-a+1} \rho \times \cdots \times \nu^{-1} \rho \times \nu^{-1} \rho \rtimes \tau_{-i},
\end{aligned}
$$

for $i=1,-1$.

Proof. Note that it follows directly from Theorem 2.1 that $\widehat{\tau}_{i}=\tau_{-i}$, for $i=1,-1$.

For an irreducible subrepresentation $\sigma$ of $\delta\left(\left[\nu^{-a} \rho, \nu^{b} \rho\right]\right) \rtimes \sigma_{\text {cusp }}$, it follows from [28, Proposition 7.5], and it is also recalled in Theorem 2.4, that there is a unique $i \in\{1,-1\}$ such that $\sigma$ is a subrepresentation of $\delta\left(\left[\nu \rho, \nu^{a} \rho\right]\right) \times$ 
$\delta\left(\left[\nu \rho, \nu^{b} \rho\right]\right) \rtimes \tau_{i}$. It follows that $\sigma$ is a subrepresentation of

$$
\nu^{b} \rho \times \cdots \times \nu^{a+1} \rho \times \nu^{a} \rho \times \nu^{a} \rho \times \cdots \times \nu \rho \times \nu \rho \rtimes \tau_{i} .
$$

Consequently, the Jacquet module of $\sigma$ with respect to an appropriate parabolic subgroup contains $\nu^{b} \rho \otimes \cdots \otimes \nu^{a+1} \rho \otimes \nu^{a} \rho \otimes \nu^{a} \rho \otimes \cdots \otimes \nu \rho \otimes \nu \rho \otimes \tau_{i}$. It follows from the properties of the Aubert involution that the Jacquet module of $\widehat{\sigma}$ with respect to an appropriate parabolic subgroup contains $\nu^{-b} \rho \otimes \cdots \otimes$ $\nu^{-a-1} \rho \otimes \nu^{-a} \rho \otimes \nu^{-a} \rho \otimes \cdots \otimes \nu^{-1} \rho \otimes \nu^{-1} \rho \otimes \tau_{-i}$ and it does not contain $\nu^{-b} \rho \otimes \cdots \otimes \nu^{-a-1} \rho \otimes \nu^{-a} \rho \otimes \nu^{-a} \rho \otimes \cdots \otimes \nu^{-1} \rho \otimes \nu^{-1} \rho \otimes \tau_{i}$.

Also, since $\tau_{i}$ is a subrepresentation of $\rho \rtimes \sigma_{\text {cusp }}$, it follows that the Jacquet module of $\sigma$ with respect to an appropriate parabolic subgroup contains $\nu^{b} \rho \otimes \cdots \otimes \nu^{a+1} \rho \otimes \nu^{a} \rho \otimes \nu^{a} \rho \otimes \cdots \otimes \nu \rho \otimes \nu \rho \otimes \rho \otimes \sigma_{\text {cusp }}$. Consequently, the Jacquet module of $\widehat{\sigma}$ with respect to an appropriate parabolic subgroup contains $\nu^{-b} \rho \otimes \cdots \otimes \nu^{-a-1} \rho \otimes \nu^{-a} \rho \otimes \nu^{-a} \rho \otimes \cdots \otimes \nu^{-1} \rho \otimes \nu^{-1} \rho \otimes \rho \otimes \sigma_{\text {cusp }}$ and $\widehat{\sigma}$ is a subrepresentation of $\nu^{-b} \rho \times \cdots \times \nu^{-a-1} \rho \times \nu^{-a} \rho \times \nu^{-a} \rho \times \cdots \times$ $\nu^{-1} \rho \times \nu^{-1} \rho \times \rho \rtimes \sigma_{\text {cusp. }}$.

Thus, there is a $j \in\{1,-1\}$ such that $\widehat{\sigma}$ is a unique irreducible (Langlands) subrepresentation of $\nu^{-b} \rho \times \cdots \times \nu^{-a-1} \rho \times \nu^{-a} \rho \times \nu^{-a} \rho \times \cdots \times \nu^{-1} \rho \times$ $\nu^{-1} \rho \rtimes \tau_{j}$. Using the Frobenius reciprocity and the above observation we deduce $j=-i$. This finishes the proof.

We also note that, for $b-a \in\{1,2\}$, it follows from [5] that the unique irreducible subrepresentation of the induced representation (5.1) is unitarizable.

The following result completes our description.

THEOREM 5.2. Suppose that $\nu^{\frac{1}{2}} \rho \rtimes \sigma_{\text {cusp }}$ reduces and let $a, b$ denote positive half-integers such that $a<b$. Let $\sigma$ denote an irreducible subrepresentation of $\delta\left(\left[\nu^{-a} \rho, \nu^{b} \rho\right]\right) \rtimes \sigma_{\text {cusp }}$. We have two possibilities.

(i) If $\mu^{*}(\sigma) \geq \delta\left(\left[\nu^{\frac{1}{2}} \rho, \nu^{a} \rho\right]\right) \times \delta\left(\left[\nu^{\frac{1}{2}} \rho, \nu^{b} \rho\right]\right) \otimes \sigma_{\text {cusp }}$, then the Aubert dual of $\sigma$ is the unique irreducible subrepresentation of

$$
\nu^{-b} \rho \times \cdots \times \nu^{-a-1} \rho \times \nu^{-a} \rho \times \nu^{-a} \rho \times \cdots \times \nu^{-\frac{1}{2}} \rho \times \nu^{-\frac{1}{2}} \rho \rtimes \sigma_{\text {cusp }} .
$$

(ii) If $\mu^{*}(\sigma)$ does not contain the irreducible constituent $\delta\left(\left[\nu^{\frac{1}{2}} \rho, \nu^{a} \rho\right]\right) \times$ $\delta\left(\left[\nu^{\frac{1}{2}} \rho, \nu^{b} \rho\right]\right) \otimes \sigma_{\text {cusp }}$, then the Aubert dual of $\sigma$ is the unique irreducible subrepresentation of

$$
\nu^{-b} \rho \times \cdots \times \nu^{-a-1} \rho \times \nu^{-a} \rho \times \nu^{-a} \rho \times \cdots \times \nu^{-\frac{3}{2}} \rho \times \nu^{-\frac{3}{2}} \rho \times \nu^{-\frac{1}{2}} \rho \rtimes S\left(\frac{1}{2}\right) .
$$

Proof. Let us first consider the case

$$
\mu^{*}(\sigma) \geq \delta\left(\left[\nu^{\frac{1}{2}} \rho, \nu^{a} \rho\right]\right) \times \delta\left(\left[\nu^{\frac{1}{2}} \rho, \nu^{b} \rho\right]\right) \otimes \sigma_{\text {cusp }} .
$$

It can be directly seen, using formula for $m^{*}$ repeteadly, that the Jacquet module of $\sigma$ with respect to an appropriate parabolic subgroup contains a 
cuspidal representation $\nu^{b} \rho \otimes \cdots \otimes \nu^{a+1} \rho \otimes \nu^{a} \rho \otimes \nu^{a} \rho \otimes \cdots \otimes \nu^{\frac{1}{2}} \rho \otimes \nu^{\frac{1}{2}} \rho \otimes \sigma_{\text {cusp }}$. Using Theorem 2.1, we directly obtain that $\widehat{\sigma}$ is a subrepresentation of

$$
\nu^{-b} \rho \times \cdots \times \nu^{-a-1} \rho \times \nu^{-a} \rho \times \nu^{-a} \rho \times \cdots \times \nu^{-\frac{1}{2}} \rho \times \nu^{-\frac{1}{2}} \rho \rtimes \sigma_{\text {cusp }},
$$

and this induced representation contains a unique irreducible subrepresentation.

Now we consider the other possibility. We obviously have an embedding

$$
\sigma \hookrightarrow \nu^{b} \rho \times \nu^{b-1} \rho \times \cdots \times \nu^{a+1} \rho \times \delta\left(\left[\nu^{-a} \rho, \nu^{a} \rho\right]\right) \rtimes \sigma_{\text {cusp }} .
$$

For a positive half-integer $x, x \geq \frac{3}{2}$, we have

$$
\begin{aligned}
\delta\left(\left[\nu^{-x} \rho, \nu^{x} \rho\right]\right) \rtimes \sigma_{\text {cusp }} & \hookrightarrow \delta\left(\left[\nu^{-x+1} \rho, \nu^{x} \rho\right]\right) \times \nu^{-x} \rho \rtimes \sigma_{\text {cusp }} \\
& \cong \delta\left(\left[\nu^{-x+1} \rho, \nu^{x} \rho\right]\right) \times \nu^{x} \rho \rtimes \sigma_{\text {cusp }} \\
& \cong \nu^{x} \rho \times \delta\left(\left[\nu^{-x+1} \rho, \nu^{x} \rho\right]\right) \rtimes \sigma_{\text {cusp }} \\
& \cong \nu^{x} \rho \times \nu^{x} \rho \times \delta\left(\left[\nu^{-x+1} \rho, \nu^{x-1} \rho\right]\right) \rtimes \sigma_{\text {cusp }}
\end{aligned}
$$

Consequently, we get that $\sigma$ is a subrepresentation of

$$
\nu^{b} \rho \times \cdots \times \nu^{a+1} \rho \times \nu^{a} \rho \times \nu^{a} \rho \times \cdots \times \nu^{\frac{3}{2}} \rho \times \nu^{\frac{3}{2}} \rho \times \delta\left(\left[\nu^{-\frac{1}{2}} \rho, \nu^{\frac{1}{2}} \rho\right]\right) \rtimes \sigma_{\text {cusp }} .
$$

In the same way as in the proof of Theorem 5.1, we obtain that there is an irreducible representation $\pi$ of some $G_{n^{\prime}}$, whose cuspidal support consists of $\nu^{\frac{1}{2}} \rho, \nu^{\frac{1}{2}} \rho$ and $\sigma_{\text {cusp }}$, such that $\widehat{\sigma}$ is a subrepresentation of

$$
\nu^{-b} \rho \times \cdots \times \nu^{-a-1} \rho \times \nu^{-a} \rho \times \nu^{-a} \rho \times \cdots \times \nu^{-\frac{3}{2}} \rho \times \nu^{-\frac{3}{2}} \rho \rtimes \pi .
$$

It remains to determine $\pi$. From its cuspidal support, we obtain that $\pi$ is a subrepresentation of one of the following induced representations: $\nu^{-\frac{1}{2}} \rho \times$ $\nu^{-\frac{1}{2}} \rho \rtimes \sigma_{\text {cusp }}, \delta\left(\left[\nu^{-\frac{1}{2}} \rho, \nu^{\frac{1}{2}} \rho\right]\right) \rtimes \sigma_{\text {cusp }}$ and $\nu^{-\frac{1}{2}} \rho \rtimes S\left(\frac{1}{2}\right)$.

Obviously, $\pi$ is not a subrepresentation of $\nu^{-\frac{1}{2}} \rho \times \nu^{-\frac{1}{2}} \rho \rtimes \sigma_{\text {cusp }}$, since this would imply that $\sigma$ is isomorphic to the other irreducible subrepresentation of $\delta\left(\left[\nu^{-a} \rho, \nu^{b} \rho\right]\right) \rtimes \sigma_{\text {cusp }}$, which is impossible.

If we assume that $\pi$ is a subrepresentation of $\delta\left(\left[\nu^{-\frac{1}{2}} \rho, \nu^{\frac{1}{2}} \rho\right]\right) \rtimes \sigma_{\text {cusp }}$, then we have $\pi \hookrightarrow \nu^{\frac{1}{2}} \rho \times \nu^{-\frac{1}{2}} \rho \rtimes \sigma_{\text {cusp }}$. Since for $x \leq-\frac{3}{2}$ we have

$$
\nu^{x} \rho \times \nu^{\frac{1}{2}} \rho \cong \nu^{\frac{1}{2}} \rho \times \nu^{x} \rho,
$$

from (5.4) we obtain that $\widehat{\sigma}$ is a subrepresentation of

$\nu^{\frac{1}{2}} \rho \times \nu^{-b} \rho \times \cdots \times \nu^{-a-1} \rho \times \nu^{-a} \rho \times \nu^{-a} \rho \times \cdots \times \nu^{-\frac{3}{2}} \rho \times \nu^{-\frac{3}{2}} \rho \times \nu^{-\frac{1}{2}} \rho \rtimes \sigma_{\text {cusp }}$.

This implies that the Jacquet module of $\sigma$ with respect to an appropriate parabolic subgroup contains

$$
\nu^{-\frac{1}{2}} \rho \otimes \nu^{b} \rho \otimes \cdots \otimes \nu^{a+1} \rho \otimes \nu^{a} \rho \otimes \nu^{a} \rho \otimes \cdots \otimes \nu^{\frac{3}{2}} \rho \otimes \nu^{\frac{3}{2}} \rho \otimes \nu^{\frac{1}{2}} \rho \otimes \sigma_{\text {cusp }},
$$

contradicting the square-integrability of $\sigma$. Thus, $\pi$ is a subrepresentation of $\nu^{-\frac{1}{2}} \rho \rtimes S\left(\frac{1}{2}\right)$ and theorem is proved. 
We also note that, for $b-a \in\{1,2\}$, it follows from [5] that a unique irreducible subrepresentation of the induced representation (5.2) and a unique irreducible subrepresentation of the induced representation (5.3) are unitarizable.

\section{REFERENCES}

[1] J. Arthur, The endoscopic classification of representations. Orthogonal and symplectic groups, American Mathematical Society, Providence, 2013.

[2] A.-M. Aubert, Dualité dans le groupe de Grothendieck de la catégorie des représentations lisses de longueur finie d'un groupe réductif p-adique, Trans. Amer. Math. Soc. 347 (1995), 2179-2189.

[3] A.-M. Aubert, Erratum: "Duality in the Grothendieck group of the category of finitelength smooth representations of a p-adic reductive group" [Trans. Amer. Math. Soc. 347 (1995), no. 6, 2179-2189; MR1285969 (95i:22025)], Trans. Amer. Math. Soc. 348 (1996), 4687-4690.

[4] W. T. Gan and L. Lomelí, Globalization of supercuspidal representations over function fields and applications, J. Eur. Math. Soc. (JEMS) 20 (2018), 2813-2858.

[5] M. Hanzer, Unitarizability of a certain class of irreducible representations of classical groups, Manuscripta Math. 127 (2008), 275-307.

[6] M. Hanzer, The unitarizability of the Aubert dual of strongly positive square integrable representations, Israel J. Math. 169 (2009), 251-294.

[7] C. Jantzen, Reducibility of certain representations for symplectic and odd-orthogonal groups, Compositio Math. 104 (1996), 55-63.

[8] C. Jantzen, Duality for classical p-adic groups: the half-integral case, Represent. Theory 22 (2018), 160-201.

[9] Y. Kim and I. Matić, Discrete series of odd general spin groups, preprint, 2018.

[10] H. Knight and A. Zelevinsky, Representations of quivers of type $A$ and the multisegment duality, Adv. Math. 117 (1996), 273-293.

[11] A. Kret and E. Lapid, Jacquet modules of ladder representations, C. R. Math. Acad. Sci. Paris 350 (2012), 937-940.

[12] I. Matić, Strongly positive representations of metaplectic groups, J. Algebra 334 (2011), 255-274.

[13] I. Matić, The conservation relation for discrete series representations of metaplectic groups, Int. Math. Res. Not. IMRN 2013, 5227-5269.

[14] I. Matić, Jacquet modules of strongly positive representations of the metaplectic group $\widetilde{S p(n)}$, Trans. Amer. Math. Soc. 365 (2013), 2755-2778.

[15] I. Matić, On discrete series subrepresentations of the generalized principal series, Glas. Mat. Ser. III 51(71) (2016), 125-152.

[16] I. Matić, On Jacquet modules of discrete series: the first inductive step, J. Lie Theory 26 (2016), 135-168.

[17] I. Matić, Aubert duals of strongly positive discrete series and a class of unitarizable representations, Proc. Amer. Math. Soc. 145 (2017), 3561-3570.

[18] I. Matić, On Langlands quotients of the generalized principal series isomorphic to their Aubert duals, Pacific J. Math. 289 (2017), 395-415.

[19] I. Matić and M. Tadić, On Jacquet modules of representations of segment type, Manuscripta Math. 147 (2015), 437-476.

[20] C. Møglin, Sur la classification des séries discrètes des groupes classiques p-adiques: paramètres de Langlands et exhaustivité, J. Eur. Math. Soc. (JEMS) 4 (2002), 143200. 
[21] C. Mœglin, Paquets stables des séries discrètes accessibles par endoscopie tordue; leur paramètre de Langlands, in: Automorphic forms and related geometry: assessing the legacy of I. I. Piatetski-Shapiro, Amer. Math. Soc., Providence, 2014, 295-336.

[22] C. Mœglin and M. Tadić, Construction of discrete series for classical p-adic groups, J. Amer. Math. Soc. 15 (2002), 715-786.

[23] C. Mœglin and J.-L. Waldspurger, Sur l'involution de Zelevinski, J. Reine Angew. Math. 372 (1986), 136-177.

[24] G. Muić, Composition series of generalized principal series; the case of strongly positive discrete series, Israel J. Math. 140 (2004), 157-202.

[25] G. Muić, On certain classes of unitary representations for split classical groups, Canad. J. Math. 59 (2007), 148-185.

[26] M. Tadić, Structure arising from induction and Jacquet modules of representations of classical p-adic groups, J. Algebra 177 (1995), 1-33.

[27] M. Tadić, On reducibility of parabolic induction, Israel J. Math. 107 (1998), 29-91.

[28] M. Tadić, On tempered and square integrable representations of classical p-adic groups, Sci. China Math. 56 (2013), 2273-2313.

[29] A. V. Zelevinsky, Induced representations of reductive p-adic groups. II. On irreducible representations of $G L(n)$, Ann. Sci. École Norm. Sup. (4) 13 (1980), 165-210.

I. Matić

Department of Mathematics

University of Osijek

Trg Ljudevita Gaja 6, 31000 Osijek

Croatia

E-mail: imatic@mathos.hr

Received: 20.3.2018.

Revised: 16.11.2018. 